\title{
Methane-Concentration and Methane-Isotope Data for Ground Water and Soil Gas in the Animas River Valley, Colorado and New Mexico, 1990-91
}

by Daniel T. Chafin, David M. Swanson, and David W. Grey

\section{U.S. GEOLOGICAL SURVEY}

Water-Resources Investigations Report 93-4007

Prepared in cooperation with the

COLORADO OIL AND GAS CONSERVATION COMMISSION,

LA PLATA COUNTY, and the

SOUTHERN UTE TRIBAL COUNCIL 


\title{
U.S. DEPARTMENT OF THE INTERIOR BRUCE BABBITT, Secretary
}

\author{
U.S. GEOLOGICAL SURVEY \\ Gordon P. Eaton, Director
}

The use of trade, product, industry, or firm names is for descriptive purposes only and does not imply endorsement by the U.S. Government.

For additional information write to:

Copies of this report can be purchased from:

District Chief

U.S. Geological Survey

Box 25046, MS 415

Denver Federal Center

Denver, CO 80225
U.S. Geological Survey

Earth Science Information Center

Open-File Reports Section

Box 25286, MS 517

Denver Federal Center

Denver, CO 80225 


\section{CONTENTS}

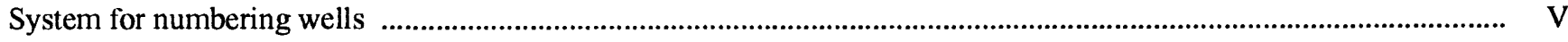

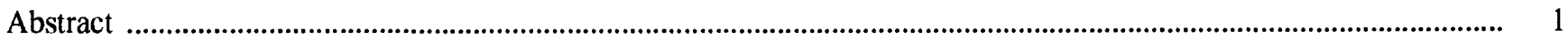

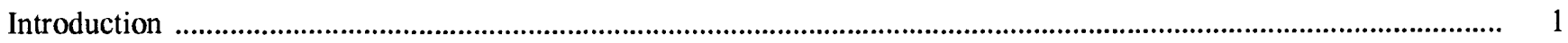

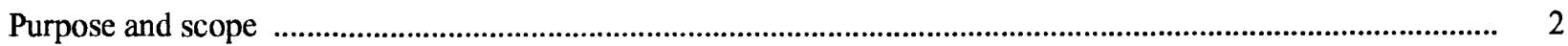

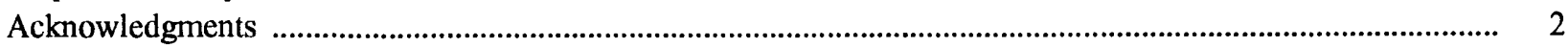

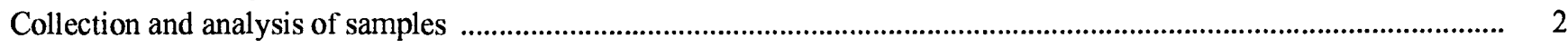

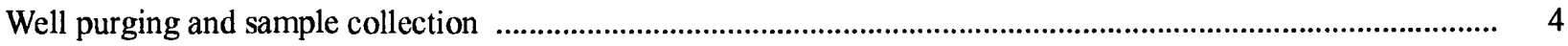

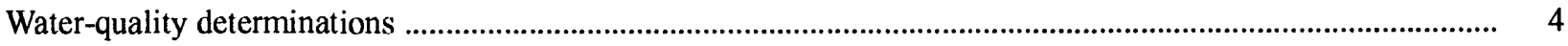

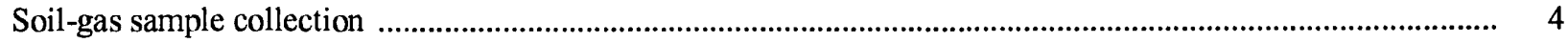

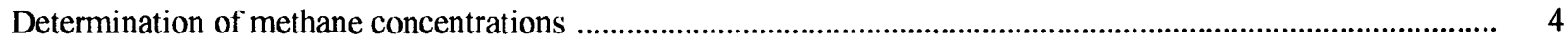

Standardization of the Organic Vapor Analyzer ................................................................................ 5

Onsite measurements of methane concentrations in water and soil samples .............................................. 5

Example calculation of methane concentration ................................................................................. 6

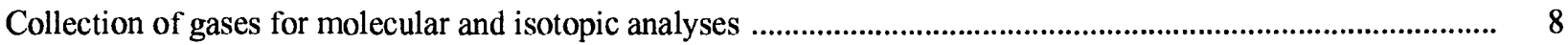

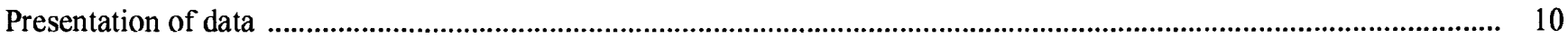

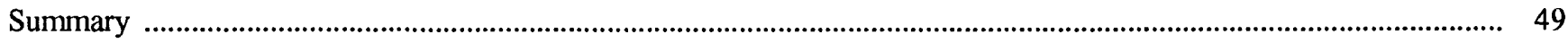

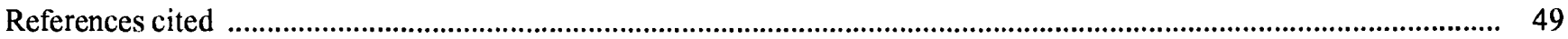

\section{PLATE}

(Plate is in pocket)

1. Map showing locations of sampled water wells and springs and soil-gas sampling sites in the Animas River Valley between Durango, Colorado, and Aztec, New Mexico

\section{FIGURES}

1. Map showing location of the study area in the San Juan Basin

2. Graph showing use of methane peak and standard calibration plot to determine mass of methane injected into Organic Vapor Analyzer

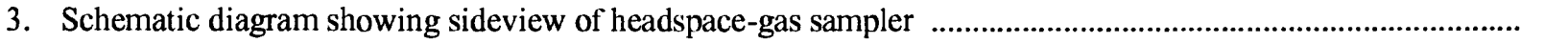

\section{TABLES}

1. Records of sampled water wells and springs

2. Water-level and water-quality measurements and nearby soil-gas-methane concentrations for selected water wells and springs

3. Gas-well records and maximum methane concentrations measured in soil gas adjacent to gas-well casings

4. Molecular-composition and methane-isotope data for gas from well water, open-field soil seeps, soil adjacent to gas-well casings, and gas-well production casings at selected sites 


$\begin{array}{lll}\text { Multiply } & \text { By } & \text { To obtain }\end{array}$

\begin{tabular}{rcl} 
atmosphere (atm) & 101.3 & kilopascal \\
centimeter $(\mathrm{cm})$ & 0.3937 & inch (in.) \\
foot (ft) & 0.3048 & meter \\
inch (in.) & 2.54 & centimeter $(\mathrm{cm})$ \\
inch (in.) & 25.40 & millimeter $(\mathrm{mm})$ \\
mile $(\mathrm{mi})$ & 1.609 & kilometer \\
liter $(\mathrm{L})$ & 0.2642 & gallon \\
liter per minute $(\mathrm{L} / \mathrm{min})$ & 0.2642 & gallon per minute \\
\hline
\end{tabular}

Degree Celsius $\left({ }^{\circ} \mathrm{C}\right)$ may be converted to degree Fahrenheit $\left({ }^{\circ} \mathrm{F}\right)$ by using the following equation:

$$
{ }^{\circ} \mathrm{F}=9 / 5\left({ }^{\circ} \mathrm{C}\right)+32
$$

Degree Fahrenheit $\left({ }^{\circ} \mathrm{F}\right)$ may be converted to degree Celsius $\left({ }^{\circ} \mathrm{C}\right)$ by using the following equation:

$$
{ }^{\circ} \mathrm{C}=5 / 9\left({ }^{\circ} \mathrm{F}-32\right)
$$

Degree Kelvin (K) may be converted to degree Celsius $\left({ }^{\circ} \mathrm{C}\right)$ by using the following equation:

$$
{ }^{\circ} \mathrm{C}=\mathrm{K}-273.15
$$

Other terms and abbreviations used in this report:

microgram $(\mu \mathrm{g})$

microgram per milliliter $(\mu \mathrm{g} / \mathrm{mL})$

micrometer $(\mu \mathrm{m})$

microsiemens per centimeter at 25 degrees Celsius $(\mu \mathrm{S} / \mathrm{cm})$

milligram (mg)

milligram per liter $(\mathrm{mg} / \mathrm{L})$

milligram per liter of gas $\left(\mathrm{mg} / \mathrm{L}_{\mathrm{g}}\right)$

milliliter $(\mathrm{mL})$

Sea level: In this report "sea level" refers to the National Geodetic Vertical Datum of 1929 (NGVD of 1929)-a geodetic datum derived from a general adjustment of the first-order level nets of both the United States and Canada, formerly called Sea Level Datum of 1929. 


\section{SYSTEM FOR NUMBERING WELLS}

Well locations (land-net locations) in this report are based on the U.S. Bureau of Land Management system of land subdivision and indicate the position of the wells by township, range, section, and position within the section. This method of well location is shown in the figure on the following page. All of the locations in this report are north of the New Mexico baseline and west of the New Mexico principal meridian.

The land-net system indicates location by using three numbers followed by two to four letters. The first number indicates the township; the second number, the range; and the next two numbers, the section in which the well is located. The letters following the section number indicate the location of the well within the section. The first letter usually denotes the quarter section; the second, the quarter-quarter section; and the third, the quarter-quarter-quarter section. The letters are assigned within the section in a counterclockwise direction, beginning with $\mathrm{A}$ in the northeastern quarter and followed by $B$ in the northwestern quarter, $C$ in the southwestern quarter, and $D$ in the southeastern quarter. Letters are assigned within each quarter section and within each quarter-quarter section in the same manner. The final letter " $X$ " indicates that the well is centered in the smallest section subdivision listed. Map locations in irregular sections were derived by alignment of the section template at the southeastern corner and along the southern boundary of the section and extending template subdivisions into uncovered section areas where necessary. A few locations for wells near Durango in Township 34 North have a " $U$ " for the first letter, which denotes a section south of the northern boundary of the Southern Ute Indian Reservation. For example, $31 \mathrm{~N}-10 \mathrm{~W}-08 \mathrm{CBD}$ indicates a well inventoried in the southeastern quarter of the northwestern quarter of the southwestern quarter of section 8, Township 31 North, Range 10 West. 


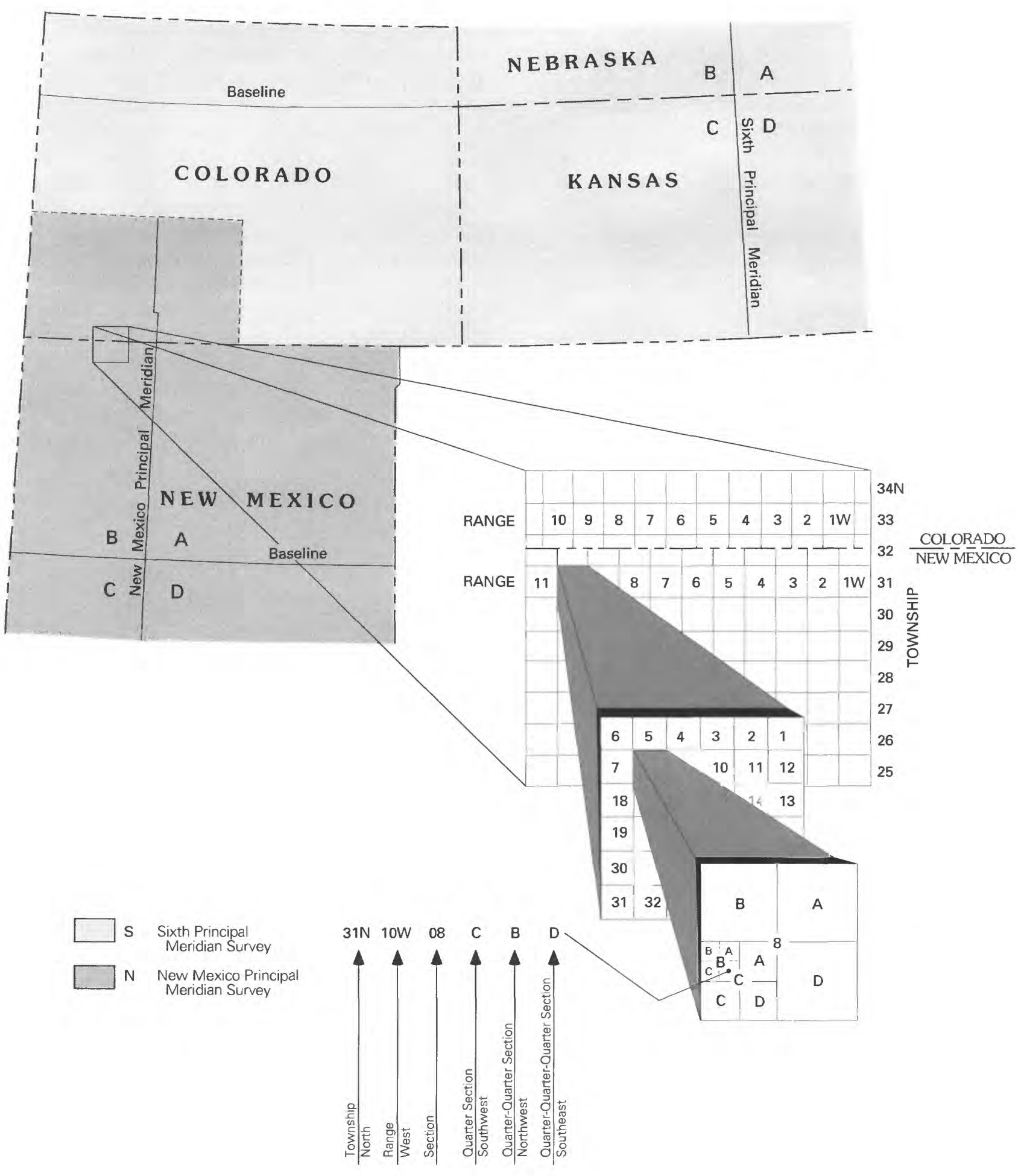




\title{
Methane-Concentration and Methane-Isotope Data for Ground Water and Soil Gas in the Animas River Valley, Colorado and New Mexico, 1990-91
}

\author{
By Daniel T. Chafin, David M. Swanson, and David W. Grey
}

\section{Abstract}

Important shallow water supplies in alluvium and Tertiary sedimentary rocks overlying Cretaceous gas-bearing formations in the San Juan Basin in Colorado and New Mexico locally are contaminated by methane. Recent development of coal-bed methane from the Cretaceous Fruitland Formation has caused public concern about the possibility of increasing contamination of shallow ground-water supplies. In July 1990, the

U.S. Geological Survey, in cooperation with the Colorado Oil and Gas Conservation Commission, La Plata County, and the Southern Ute Tribal Council, Colorado, began a study in the Animas River Valley between Durango, Colorado, and Aztec, New Mexico. The purpose of this study is to map the occurrence of methane in shallow ground water and to identify possible sources of and migration pathways for methane. This report addresses the data-collection phase of the study.

This report presents data collected by the U.S. Geological Survey mostly during August 1990-May 1991. These data consist of:

(1) Records of water wells and gas wells;

(2) water-quality data, including methane concentrations, from wells and springs in the Animas

River Valley; (3) concentrations of methane in soil gas near water wells and springs and adjacent to gas-well casings within about one-half mile of the Animas River Valley; and (4) molecular composition and methane-isotope data for gas samples collected from ground-water headspace, soil, and gas-well production casings.

Water-quality data consist of onsite and laboratory measurements of concentrations of methane and other dissolved constituents in water samples collected from wells and springs. Seventy-one wells and 1 spring in Colorado were sampled, and 132 wells and 1 spring in New Mexico were sampled. Onsite measurements were made for determination of specific conductance, $\mathrm{pH}$, temperature, and dissolvedmethane concentrations. At 68 selected sites, additional water samples were collected for onsite determination of alkalinity and laboratory determinations of calcium, magnesium, sodium, potassium, sulfate, chloride, bromide, silica, iron, and manganese concentrations. Onsite methaneconcentration measurements of soil-gas and water samples were made with a portable organic gas analyzer equipped with a chromatographic column. Dissolved-methane concentrations were determined by measuring the concentration of methane in headspace gas.

Soil-gas concentrations were measured near 192 ground-water sites and adjacent to the casings of 352 gas wells. Gas was collected from 16 water samples, from 3 soil seeps in open fields, from 10 soil columns adjacent to gas-well casings and 1 soil column adjacent to a cathodic-protection well, and from 30 gas wells. These samples were analyzed for molecular composition and carbon-13 content of methane. Seventeen of these samples were selected for analysis of deuterium content of methane.

\section{INTRODUCTION}

Shallow ground water is locally contaminated with methane in the northwestern part of the San Juan Basin where aquifers are underlain by gas-bearing Cretaceous rocks. Important domestic water supplies are obtained from alluvium and Tertiary sedimentary rocks overlying these gas-bearing rocks. Recent development (especially during the late 1980's) of coal-bed methane from the Fruitland Formation in the San Juan Basin has caused public concern about the possibility of increasing methane concentrations in shallow ground water. The Animas River Valley, one of the most populated areas in the San Juan Basin, is underlain by productive gas fields in the Dakota Sandstone, Mesaverde Group, Pictured Cliffs Sandstone, and the Fruitland Formation. 
In July 1990, the U.S. Geological Survey (USGS), in cooperation with the Colorado Oil and Gas Conservation Commission, La Plata County, and the Southern Ute Tribal Council, began a study of methane in shallow ground water beneath the Animas River Valley between Durango, Colorado, and Aztec, New Mexico (fig. 1).

The purpose of this study is to investigate possible sources of and migration pathways for methane contamination of shallow ground water in the study area. The specific objectives of the study are to:

1. Map the presence of methane in shallow ground water;

2. Attempt to differentiate among areas where increased methane concentrations result from leaking gas wells, areas where increased methane concentrations result from upward movement through geologic units and fractures, and areas where methane is produced within shallow aquifers;

3. Assess the current chemical quality of potable ground water and evaluate the potential for contamination by upward movement of water with large dissolved-solids concentrations; and

4. Qualitatively describe ground-water flow between the Fruitland Formation and nearsurface water supplies (shallow aquifers and surface water).

\section{Purpose and Scope}

This report addresses the data-collection phase of this study and presents data collected by the U.S. Geological Survey, mostly during August 1990May 1991. These data consist of: (1) Information about location and construction of sampled water wells, springs, and gas wells; (2) water-quality determinations, including methane concentrations, from domestic wells and springs in or adjacent to the Animas River Valley; (3) concentrations of methane in soil gas near water wells and adjacent to gas-well casings within about $0.5 \mathrm{mi}$ of the Animas River Valley; and (4) molecular and methane-isotope data for gases from ground water, soil, and gas wells. The locations of sampling sites are shown on plate 1 .

\section{Acknowledgments}

The authors appreciate the cooperation of homeowners in the study area who allowed access to their property and thank the Aztec District of the New Mexico Oil Conservation Commission for the information used in the construction of the gas-well data base. In addition, the authors acknowledge the Gas Research Institute and the Amoco Production Company for providing supplementary information that was useful in this study.

\section{COLLECTION AND ANALYSIS OF SAMPLES}

Results of analyses of water samples from 203 wells and 2 springs were used to map the presence of methane in ground water, to determine relations between methane concentrations and other factors, and to select water samples for molecular and methaneisotope analyses of headspace gas. Information about sampled wells and springs was obtained from well or spring owners when they were contacted for permission to sample. This information was checked against permits filed with the Colorado and New Mexico State Engineer offices. Permits were not available for many sampled wells and springs; therefore, the reliability of construction data for these wells could not be verified. The primary aquifer tapped by a well was determined from information provided by owners, well logs (if available), and field observations.

Most ground-water samples were collected from wells used to obtain drinking water and water for yard irrigation and livestock. Piped public-supply water is available in the study area between Aztec and Cedar Hill, New Mexico, although many homeowners have opted not to use it. One public-supply well and two springs were sampled. Samples were collected at as many sites as possible in areas where wells were sparse and at selected sites in areas where wells were distributed more densely. Because the well distribution is denser in the New Mexico part of the study area than in the Colorado part, more wells were sampled in the New Mexico part of the study area than in the Colorado part. Seventy-one water wells and 1 spring in Colorado were sampled, and 132 water wells and 1 spring in New Mexico were sampled. Initial sampling was done during August-October 1990. Water from 20 sites in the Cedar Hill, New Mexico, area was resampled randomly during February 20-25, 1991. The purpose of resampling was to test seasonal effects on variations in concentrations of methane in ground water. 


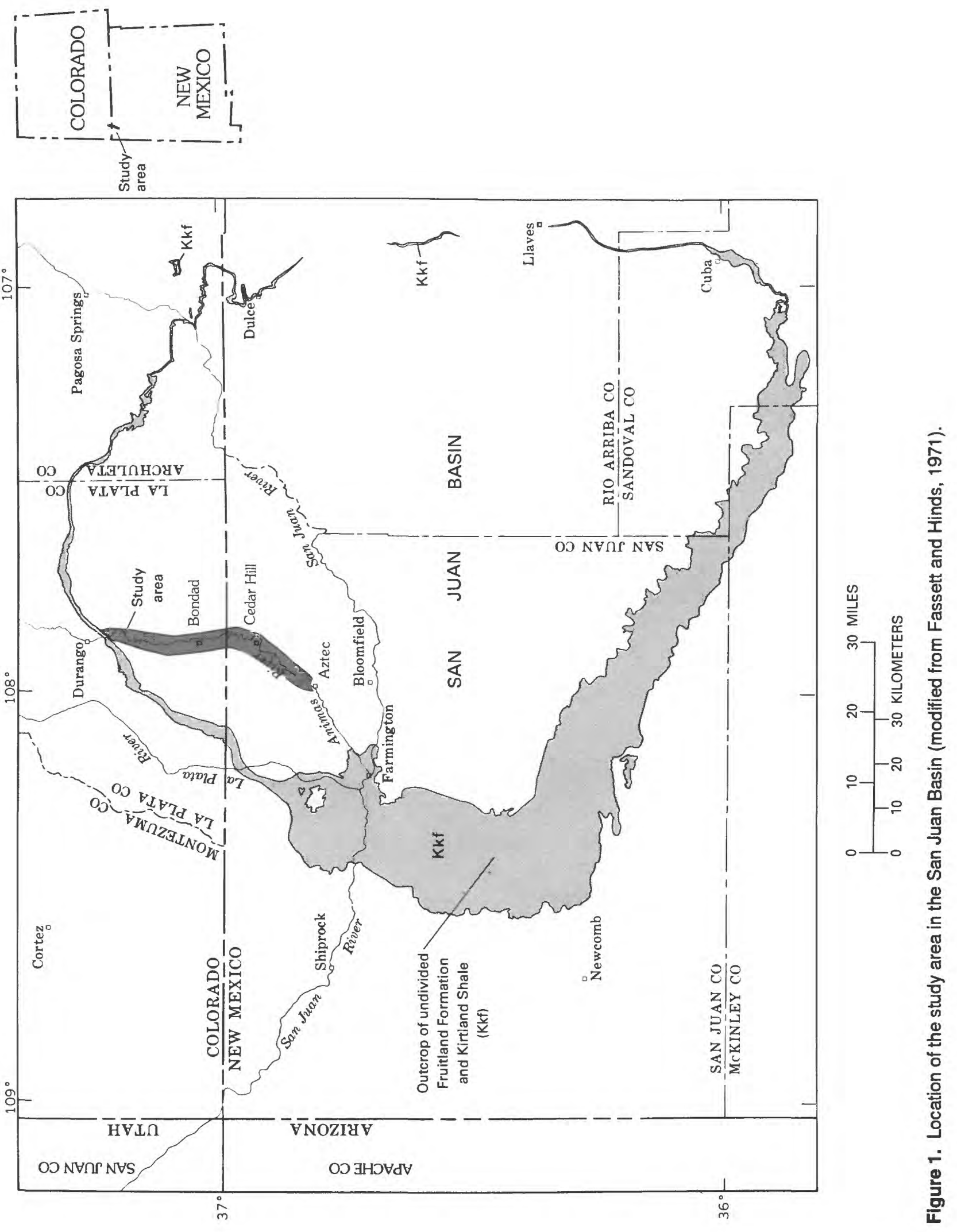


Soil-gas-methane concentrations were measured at 192 ground-water sites, by the casings of 352 gas wells (and some associated cathodic-protection wells) within $0.5 \mathrm{mi}$ of the Animas River Valley, and at 4 open-field soil seeps. The purpose of these measurements was to determine relations between soilgas-methane concentrations and dissolved-methane concentrations at water-quality sites, between soilgas-methane concentrations at water-quality sites and gas wells, and to select soil-gas samples for molecular and methane-isotope analyses.

Gas from 16 water samples, from 3 soil seeps in open fields, from 10 soil columns adjacent to gas-well casings and 1 column adjacent to a cathodic-protection well, and from 30 gas wells were collected for analyses of molecular composition and carbon- 13 content of methane. The purpose of this sampling was to provide information about possible sources of methane in shallow ground water and soil.

\section{Well Purging and Sample Collection}

Before water-quality samples were collected, each water well was prepumped to purge the casing of standing water. At least one casing volume of water was prepumped. Casing volume was estimated from well depth and casing diameter at the surface. Water was pumped and collected from outside faucets that were as close as possible to the well. The timed discharge rate was used to calculate the time required to prepump one casing volume from the well.

\section{Water-Quality Determinations}

Following prepumping, water samples were collected from the same outside faucet for onsite measurements and laboratory analyses. Specific conductance, $\mathrm{pH}$, temperature, and dissolved-methane concentrations were measured onsite for each water sample. Laboratory analyses of 68 selected water samples were made for determinations of concentrations of major dissolved ions and selected trace elements. These determinations were for calcium, magnesium, sodium, potassium, sulfate, chloride, bromide, silica, iron, and manganese. Samples for all of these determinations were filtered through a cellulose acetate filter with $0.45-\mu \mathrm{m}$ pores. Samples for all determinations except sulfate, chloride, and bromide were stabilized with nitric acid. Laboratory analyses were done at the USGS National Water-Quality Laboratory in Arvada, Colorado, according to methods described in Fishman and Friedman (1989). Incremen- tal alkalinity titrations were performed onsite for samples collected for laboratory determinations.

\section{Soil-Gas Sample Collection}

Soil-gas samples were collected by inserting a perforated steel tube 3 to $4 \mathrm{ft}$ into the ground. Soil gas was withdrawn from the tube by means of a small battery-operated vacuum pump. After prepumping for 1 minute to purge the tube, a gas sample was withdrawn with a gas-tight syringe and immediately injected into a gas chromatograph.

Soil-gas samples at ground-water sites generally were collected 50 to $100 \mathrm{ft}$ downvalley from the well or spring. Soil-gas samples generally were collected within $1 \mathrm{ft}$ of gas-well and cathodic-protection-well casings. Generally, several samples were collected from various locations around the casings, and the maximum concentrations are reported.

\section{Determination of Methane Concentrations}

Methane concentrations were measured onsite with a Century OVA-128GC Organic Vapor Analyzer (OVA) equipped with a 24-in., Porapak-N, 80/100-mesh gas-chromatograph column capable of separating methane, ethane, and heavier hydrocarbon gases. The incorporated detector is the flame-ionization type. Hydrogen gas was the carrier gas and the fuel supply. Methane eluted in about 20 seconds following injection, whereas ethane eluted in about $2 \mathrm{~min}$ utes at $75^{\circ} \mathrm{F}$. Actual elution times varied with ambient temperatures in the field environment. Chromatograms were recorded with an accessory chart recorder.

The chromatograph signal response varied slightly with onsite conditions. Calibrations indicated that the response was 10 percent greater at $25^{\circ} \mathrm{F}$ than at $75^{\circ} \mathrm{F}$. Because most ground-water analyses were done between 50 to $85^{\circ} \mathrm{F}$, the actual response variability caused by temperature variations probably was less than 10 percent. The response also varied with ambient atmospheric pressure. However, this variation probably was small because the difference in elevation between the highest ground-water-sample site $(6,470 \mathrm{ft})$ and the lowest ground-water-sample site $(5,610 \mathrm{ft})$ was small. This elevation difference corresponds to about a 3-percent difference in mean barometric pressure; pressure variation because of weather changes is similar. Onsite calibration checks indicated that the overall variation in accuracy was about \pm 20 percent. This variation is acceptable for analyses done with a field instrument. 


\section{Standardization of the Organic Vapor Analyzer}

The OVA was standardized with a 95-partsper-million (volume-to-volume basis, \pm 5 percent) methane-in-air standard obtained commercially. A 1-L, Teflon gas-sampling bag was filled with this standard gas to ambient atmospheric pressure. Ambient atmospheric pressure was established by use of a bleed tube connected to the gas-supply line. After the bag was slightly overpressured, the standard tank was closed, and the clamp on the bleed tube was opened, which allowed excess pressure to bubble the methane under about $1 \mathrm{~cm}$ of water until bubbling stopped. The clamp was closed, and atmospheric pressure was determined with a barometer and recorded. Ambient temperature was determined with a thermometer and recorded.

The Ideal Gas Law was used to calculate the concentration of methane in the gas-sampling bag:

$$
P^{\prime} V=n R T
$$

where

$P^{\prime}=$ partial pressure of methane, in atmospheres;

$V=$ volume, in milliliters;

$n$ = moles of methane; and

$R=$ gas constant $\left(82.06 \mathrm{~atm} \mathrm{~mL} \mathrm{~mole}{ }^{-1} \mathrm{~K}^{-1}\right)$;

$T=$ temperature, in degrees Kelvin.

The following equations were substituted into equation (1) to provide the appropriate units and the partial pressure of the 95-parts-per-million methane standard:

$$
P^{\prime}=(P)\left(95 \times 10^{-6} \mathrm{~mL} \mathrm{CH}_{4} / \mathrm{mL}\right)
$$

and

$$
n=m / M
$$

where,

$P=$ ambient pressure, in atmospheres; and

$m$ = mass of methane, in milligrams;

$M=$ molecular weight of methane

(16,040 milligrams per mole).

These substitutions, rearrangement, and use of the appropriate units yield the final equation for the concentration of methane, $C_{s}$ (in micrograms per milliliter), in the sample bag:

$$
C_{s}=m / V=18.57 \frac{P}{T}
$$

The OVA was calibrated by means of three injections of each of the following volumes of the standard gas: $0.050,0.100,0.250,0.500$, and $0.750 \mathrm{~mL}$. The mass of methane injected, $m_{s}$ (in micrograms), for each of these volumes, $v_{s}$ (in milliliters), was calculated by:

$$
\begin{gathered}
C_{s}=m_{s} / v_{s} \\
\quad \text { or } \\
m_{s}=C_{s} v_{s}
\end{gathered}
$$

Average peak areas were plotted against the masses of methane injected to generate a standard calibration plot.

\section{Onsite Measurements of Methane Concentrations in Water and Soil Samples}

Peak areas that resulted from injection into the OVA of known volumes of gas from headspaces over water samples or from soil columns, $v_{s}$ (in milliliters), were used to determine concentrations of methane.

The peak area of the sample methane and standard calibration plot were used to determine the mass of methane, $m_{s}$ (in micrograms), in a gas sample injected into the OVA. The concentration of methane in the sample, $C_{s}$ (in milligrams per liter), was then calculated using equation (5) or, if a 1-mL sample aliquot was diluted 43 times by injection into a $43-\mathrm{mL}$ vial, then

$$
C_{s}=\left(m_{s} / v_{s}\right)(43 \mathrm{~mL} / 1 \mathrm{~mL})
$$

Some soil-gas samples required even larger dilution factors. Those factors were substituted for 43 in equation (6).

Dissolved-methane concentration was determined by analyzing headspace gas and by calculating the concentration of methane remaining in the water sample (assuming equilibration). The steps involved in this procedure were as follows:

1. Two 43-mL septum vials (one for backup) were quickly filled with gently flowing sample water and immediately capped without air-bubble inclusion.

2. A $20-\mathrm{mL}$ headspace was produced in each vial by injecting $20 \mathrm{~mL}$ of air into the upside end of the inverted vial with a $20-\mathrm{mL}$ glass syringe tipped with a 3.5-in., 18-gage needle and allowing water to be ejected from the vial through a 1.5-in., 18-gage needle inserted about 0.1 in. into the downside, septum-capped end of the vial.

3. Each vial was shaken gently and laid on its side (at a small angle to prevent headspace contact with the septum) for 20 minutes, during which 
time it was shaken occasionally; ambient temperature was recorded. This duration was assumed to be long enough to allow equilibrium to develop between methane concentrations in dissolved and headspace-gas phases.

4. A volume of headspace gas, $v_{s}(0.5 \mathrm{~mL}$ or less $)$, was extracted from a vial and injected into the OVA. Larger methane concentrations caused off-scale peak heights and required dilution of the sample and another injection into the OVA. For example, a 43-times dilution was accomplished by injecting $1 \mathrm{~mL}$ of headspace gas into a 43-mL vial filled with ambient air.

5. The resulting methane peak area and the standard calibration plot were used to determine the mass of injected methane, $m_{s}$. The headspace concentration of methane, $C_{s}$ (in milligrams per liter), was determined by using equation (5) or (6) or equation (6) with the appropriate dilution factor substituted for larger dilutions.

6. The partial pressure of methane in the headspace, $P^{\prime}$, was determined by substituting equation (3) into equation (1), rearranging, and substituting specific sample variables, $m_{s}$ and $v_{s}$,

$$
P^{\prime}=m_{s} R T / M v_{s}=C_{s} R T / M
$$

7. The aqueous methane concentration, $C^{\prime}$ (in milligrams per liter), at assumed equilibrium with the headspace methane concentration in the vial was determined from the partial pressure of methane in the headspace, $P^{\prime}$, and the Bunsen solubility coefficient, $\beta_{T}$. $\beta_{T}$ expresses the volume of methane in milliliters (normalized to conditions of 1 atm partial pressure of methane and $273.15 \mathrm{~K}$ ) soluble in $1 \mathrm{~mL}$ of water at a given temperature and at equilibrium with a partial pressure of methane of $1 \mathrm{~atm}$ (Yamamoto and others, 1976, p. 80). Freshwater Bunsen coefficients tabulated for temperatures from $0^{\circ} \mathrm{C}$ to $30^{\circ} \mathrm{C}$ were obtained from Yamamoto and others (1976, p. 80). By using equation (7), a concentration of $715.6 \mathrm{mg} / \mathrm{L}_{\mathrm{g}}$ is obtained for the normalized $\beta_{T}$ conditions of $1 \mathrm{~atm}$ partial pressure of methane and $273.15 \mathrm{~K}$. Henry's law states that at equilibrium the aqueous concentration of a relatively insoluble gas is directly proportional to its partial pressure (Freeze and Cherry, 1979, p. 95).
Therefore, the concentration of dissolved methane in the vial $\left(C^{\prime}\right)$ is a function of the partial pressure of methane in the headspace and the Bunsen solubility coefficient for the temperature in the vial:

$$
C^{\prime}=715.6 \mathrm{P}^{\prime} \beta_{T} \mathrm{mgL}^{-1} \mathrm{~atm}^{-1}
$$

8. The concentration of methane in the $20-\mathrm{mL}$ volume of headspace, $C_{\mathrm{s}}$, was exsolved from the $23 \mathrm{~mL}$ of water. $C^{\prime}$ represents the concentration of methane remaining in the $23 \mathrm{~mL}$ of water after equilibration. Therefore, the concentration of methane (in milligrams per liter) in the original water sample, $C_{W}$ must be corrected for the quantity of methane lost to the headspace by using:

$$
C_{W}=\frac{C_{s}(20 \mathrm{~mL})+C^{\prime}(23 \mathrm{~mL})}{23 \mathrm{~mL}}
$$

\section{Example Calculation of Methane Concentration}

Measurement of the dissolved-methane concentration in water from a well at 33N-10W-13DDC can be used to illustrate the use of the standard calibration plot for the OVA and equations (6) through (9). After creation of a $20-\mathrm{mL}$ headspace in the $43-\mathrm{mL}$ sample vial and a 20 -minute wait, $1 \mathrm{~mL}$ of headspace gas at $28^{\circ} \mathrm{C}(301 \mathrm{~K})$ was extracted with a gas-tight syringe and injected into a 43-mL vial filled with ambient air. A $0.050-\mathrm{mL}\left(v_{s}\right)$ aliquot was extracted from this dilution vial and was injected into the OVA. The resulting methane peak is shown in the inset for figure 2. A nominal peak area (actually twice the peak area) was calculated by multiplying the base width ( 2.5 units) by the base height (21.5 units) to obtain 54 squared units. Use of this nominal peak area and the calibration plot (fig. 2) indicates that $0.024 \mu g\left(m_{s}\right)$ of methane was injected into the OVA. Equation (6) was used to determine the concentration of methane in the undiluted headspace gas $\left(C_{s}\right)$ :

$$
\begin{gathered}
C_{s}=\left(m_{s} / v_{s}\right)(43 \mathrm{~mL} / 1 \mathrm{~mL}) \\
C_{s}=(0.024 \mu \mathrm{g} / 0.050 \mathrm{~mL})(43) \\
C_{s}=21 \mu \mathrm{g} / \mathrm{mL}(\text { or } \mathrm{mg} / \mathrm{L}) .
\end{gathered}
$$

The method of calculation through this step applies to either a headspace-gas or a soil-gas concentration. The remaining steps apply only to calculation of the concentration of methane in a water sample as collected. 


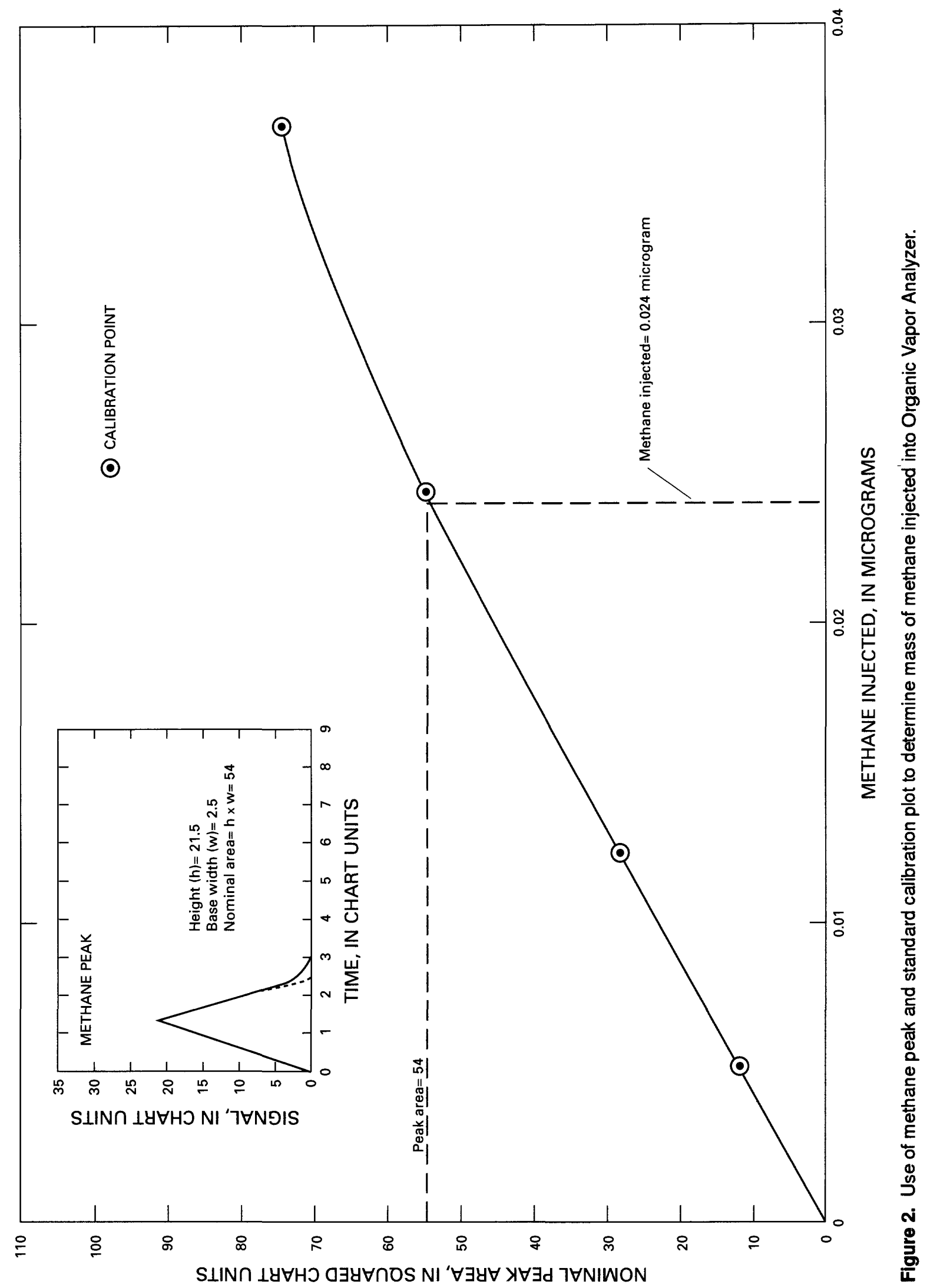


The partial pressure of methane in the undiluted headspace gas $\left(P^{\prime}\right)$ was determined for $28^{\circ} \mathrm{C}(301 \mathrm{~K})$ by using $C_{s}$ and equation (7).

$$
\begin{gathered}
P^{\prime}=C_{s} R T / M \\
P^{\prime}=\frac{\left(21 \mathrm{mg} \mathrm{L}^{-1}\right)\left(82.06 \mathrm{~atm} \mathrm{~mL} \mathrm{~mole} \mathrm{~K}^{-1}\right)(301 \mathrm{~K})}{\left(16,040 \mathrm{mg} \mathrm{mole}-1,000 \mathrm{~mL} \mathrm{~L}^{-1}\right)} \\
P^{\prime}=0.032 \mathrm{~atm} .
\end{gathered}
$$

The partial pressure of methane $\left(P^{\prime}\right)$, the Bunsen coefficient for $28^{\circ} \mathrm{C}\left(\beta_{28^{\circ} \mathrm{C}}=0.02994\right)$, and equation (8) were used to calculate the concentration of dissolved methane at equilibrium with the headspace gas $\left(C^{\prime}\right)$ :

$$
\begin{gathered}
C^{\prime}=715.6 P^{\prime} \beta_{T} \mathrm{mg} \mathrm{L}^{-1} \cdot \mathrm{atm}^{-1} \\
C^{\prime}=715.6(0.032 \mathrm{~atm})(0.02994) \mathrm{mg} \mathrm{L}^{-1} \mathrm{~atm}^{-1} \\
C^{\prime}=0.69 \mathrm{mg} \mathrm{L}^{-1} .
\end{gathered}
$$

Finally, the concentration of methane in the water sample as collected $\left(C_{W}\right)$ was calculated by using $C_{s}$ and $C^{\prime}$ in equation 9 :

$$
\begin{gathered}
C_{W}=\frac{C_{s}(20 \mathrm{~mL})+C^{\prime}(23 \mathrm{~mL})}{23 \mathrm{~mL}} \\
C_{W}=\frac{21 \mathrm{mg} \mathrm{L}^{-1}(20 \mathrm{~mL})+0.69 \mathrm{mg} \mathrm{L}^{-1}(23 \mathrm{~mL})}{23 \mathrm{~mL}} \\
C_{W}=19 \mathrm{mg} \mathrm{L}^{-1}
\end{gathered}
$$

\section{Collection of Gases for Molecular and Isotopic Analyses}

Gas from ground water was collected for determination of molecular and isotopic composition by means of a dynamic headspace-gas sampler. This sampler consisted of an 800-mL, cylindrical, clear plastic chamber with water-inflow and gas-collection ports at the top and a water-outflow port at the bottom (fig. 3). In addition, a syringe-septum port was mounted at the top to allow measurement of the concentration of methane in the headspace. Water was pumped into the chamber, and a headspace volume of about $100 \mathrm{~mL}$ was produced by stopping the flow, unclamping the tube connected to the gas-collection port, and allowing some water to drain. The tube was reclamped, and flow was resumed at a rate of about $2 \mathrm{~L} / \mathrm{min}$, which caused substantial turbulence and bubbling in the chamber. Periodically, samples of headspace gas were extracted by syringe from the septum port and injected into the por- table gas chromatograph to determine the approximate concentration of methane. This concentration was used to determine whether the headspace gas contained sufficient methane ( 5 percent) for reliable laboratory determination of the carbon-13 content of methane. A dissolved concentration of about $2 \mathrm{mg} / \mathrm{L}$ generally was required to obtain sufficient headspace concentration. After sufficient methane was present in the headspace (generally 30 to 60 minutes), the headspace gas was collected in a $473-\mathrm{mL}$ glass bottle having a bracketmounted top and a rubber seal ring. The bottle previously had been filled with fresh sample water. Collection was done by opening the gas-collection port and by restricting the flow of water through the water-outflow port by pinching the water-outflow hose, thereby forcing the headspace gas through a tube into the sample bottle, which was inverted and submerged under water. About $0.1 \mathrm{~mL}$ of 17 -percent benzalkonium chloride biocide was added with a syringe to the water remaining in the bottle to prevent bacterial degradation of gases, and the bottle was sealed under water to minimize contamination by ambient air.

Soil-gas samples from gas wells and the cathodic-protection well generally were collected within $1 \mathrm{ft}$ of the casing. Samples were collected first through a perforated steel tube inserted 3 to $4 \mathrm{ft}$ in the ground as described in the section "Soil-Gas Sample Collection." The methane concentration was measured as described in the subsection "Onsite Measurements for Water and Soil Samples." If sufficient methane was present for reliable chemical and isotopic analysis, a sample was taken by allowing a water-filled sample bottle to drain through tubing almost completely, which drew soil gas into the bottle through another tube connected to the soil-gas-sampling tube. Biocide was added, and the bottle was sealed while inverted under water.

Production gas from gas wells generally was collected from valved ports in the gage house. Several gas wells were not producing; however, gas from those wells was collected from the production tubes on the casing heads. Gas was sampled by allowing it to blow through a tube into a water-filled sample bottle that was inverted and submerged under water. The sample was preserved and sealed as described above.

All gas samples were analyzed for chemical content and methane carbon-13 content by the U.S. Geological Survey in Denver, Colorado. Concentrations of air components, hydrocarbons, and carbon dioxide were determined by means of thermal-conductivity gas chromatography. The methane was collected from a port and converted to carbon dioxide in a vacuum combustion system. The stable carbon isotopic ratios of the carbon dioxide were measured by means of mass spec- 


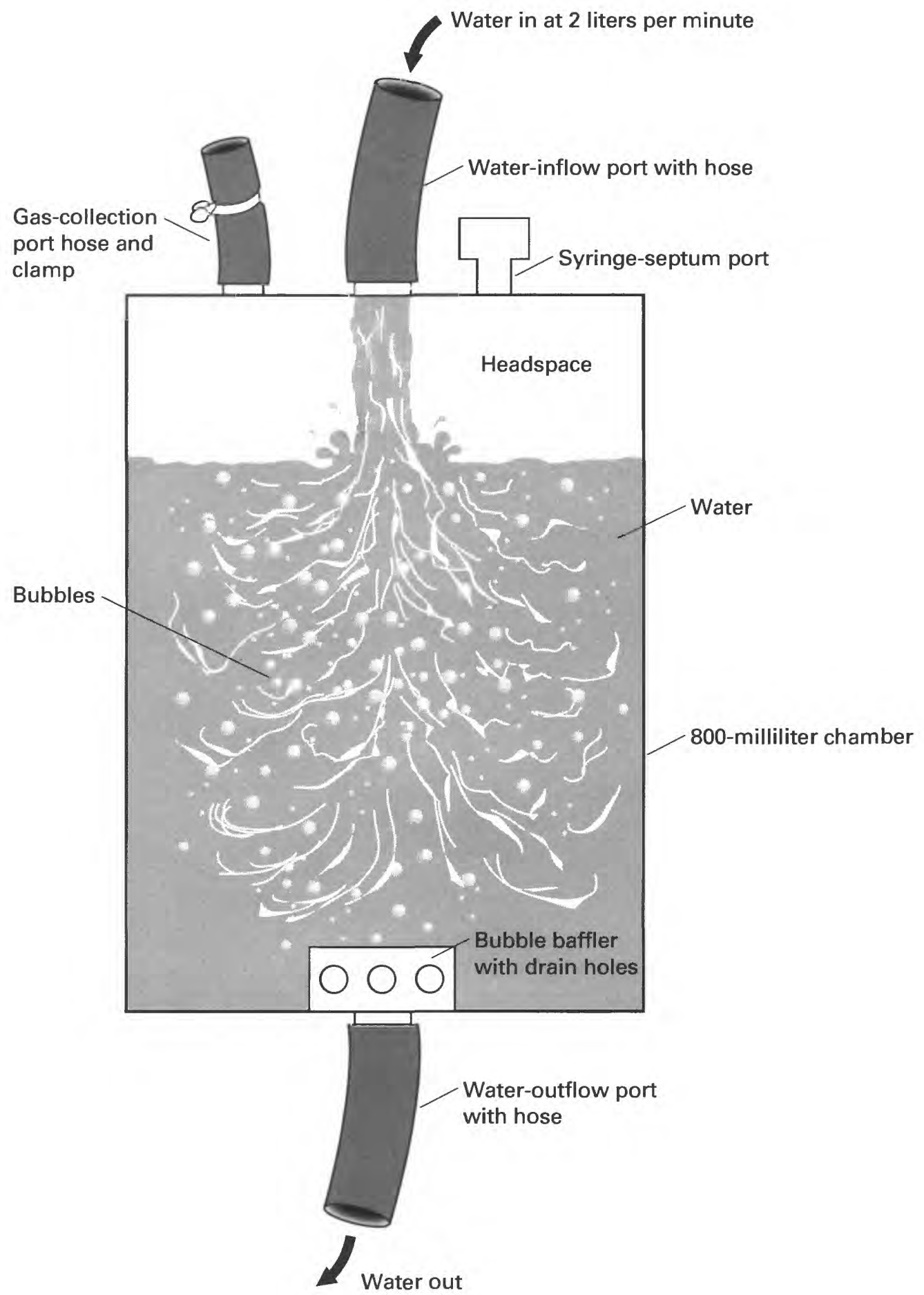

Figure 3. Schematic diagram showing sideview of headspace-gas sampler. 
trometry. These ratios are reported in the $\partial$-notation per thousand (per mil) deviations, relative to the Peedee belemnite marine carbonate standard. Selected samples were analyzed by a contract laboratory using similar methods to determine methane hydrogenisotope ratios. These ratios are reported in $\partial$-notation per thousand deviations, relative to the Standard Mean Ocean Water (SMOW) standard.

\section{PRESENTATION OF DATA}

Well location and construction information for selected water wells and springs are listed in table 1. Results for water-level and water-quality measurements for these wells and springs and results for nearby soil-gas measurements are listed in table 2. Gas-well information and maximum concentrations of methane measured in soil gas adjacent to gas-well casings and some cathodic-protection wells are listed in table 3 . Molecular-composition and methane-isotope data for gas from water, open-field soil seeps, soil adjacent to gas-well casings, and gas wells are listed in table 4. 
Tabie 1. Records of sampled water wells and springs

[Depths of wells are in feet below land surface; altitudes of land surface are shown in feet above sea level; --, no data; Fm, Formation; -, year approximate]

\begin{tabular}{|c|c|c|c|c|c|c|c|}
\hline $\begin{array}{c}\text { Number } \\
\text { on } \\
\text { plate } 1\end{array}$ & $\begin{array}{l}\text { Well or spring } \\
\text { owner }\end{array}$ & Station number & Land-net location & $\begin{array}{l}\text { Year } \\
\text { drilled }\end{array}$ & $\begin{array}{c}\text { Total } \\
\text { depth } \\
\text { of } \\
\text { well }\end{array}$ & $\begin{array}{l}\text { Altitude } \\
\text { of land } \\
\text { surface }\end{array}$ & Aquifer \\
\hline 1 & James Rosenau & 371312107512701 & $34 \mathrm{~N}-09 \mathrm{~W}-09 \mathrm{DBC}$ & -- & 25 & 6,370 & Animas Fm \\
\hline 2 & Jim Sinton & 371253107511801 & $34 \mathrm{~N}-09 \mathrm{~W}-09 \mathrm{DCD}$ & -- & 180 & 6,400 & Animas Fm \\
\hline 3 & $\begin{array}{l}\text { Colorado Ute Elec- } \\
\text { tric Association }\end{array}$ & 371242107512901 & $34 \mathrm{~N}-09 \mathrm{~W}-07 \mathrm{AAA}$ & 1985 & 199 & 6,440 & Animas Fm \\
\hline 4 & Charles Weekly & 371225107513801 & $34 \mathrm{~N}-09 \mathrm{~W}-07 \mathrm{ADC}$ & 1983 & 300 & 6,470 & Animas Fm \\
\hline 5 & David Wylie & 371211107514601 & $34 \mathrm{~N}-09 \mathrm{~W}-07 \mathrm{DBD}$ & 1983 & 200 & 6,450 & Animas Fm \\
\hline 6 & Roy Peterson & 371204107515001 & $34 \mathrm{~N}-09 \mathrm{~W}-07 \mathrm{DCA}$ & 1985 & 380 & 6,450 & Animas Fm \\
\hline 7 & Jolius Roastinger & 371148107520701 & $34 \mathrm{~N}-09 \mathrm{~W}-18 \mathrm{BAA}$ & 1984 & 262 & 6,420 & Animas Fm \\
\hline 8 & Peter Kewitt & 371127107521701 & $34 \mathrm{~N}-09 \mathrm{~W}-18 \mathrm{CAB}$ & 1978 & 162 & 6,310 & Animas Fm \\
\hline 9 & Glenn Wycoff & 371126107522401 & $34 \mathrm{~N}-09 \mathrm{~W}-18 \mathrm{CBA}$ & 1977 & 110 & 6,330 & Animas Fm \\
\hline 10 & Robin Converse & 371013107523001 & $34 \mathrm{~N}-09 \mathrm{~W}-19 \mathrm{CCC}$ & 1984 & 190 & 6,260 & Animas Fm \\
\hline 11 & Tom Joerger & 371002107522801 & $34 \mathrm{~N}-09 \mathrm{~W}-30 \mathrm{BBC}$ & 1987 & 73 & 6,220 & Valley fill \\
\hline 12 & Arabella Williams & 370852107525301 & $34 \mathrm{~N}-10 \mathrm{~W}-36 \mathrm{ACD}$ & 1983 & 136 & 6,240 & Animas Fm \\
\hline 13 & LaDonna Bellman & 370852107522701 & $34 \mathrm{~N}-09 \mathrm{~W}-31 \mathrm{BCC}$ & 1981 & 126 & 6,240 & Animas Fm \\
\hline 14 & Ron Ollier & 370848107531801 & $34 \mathrm{~N}-10 \mathrm{~W}-36 \mathrm{CAB}$ & -- & 250 & 6,200 & Animas Fm \\
\hline 15 & Mark Goldfarb & 370847107530701 & $34 \mathrm{~N}-10 \mathrm{~W}-36 \mathrm{CAA}$ & 1965 & 69 & 6,200 & Animas Fm \\
\hline 16 & $\begin{array}{l}\text { Rainbow Springs } \\
\text { Trout Ranch }\end{array}$ & 370836107520801 & $34 N-09 W-31 C D B$ & -- & 0 & 6,400 & Terrace deposits \\
\hline 17 & R.L. Robertson & 370804107524701 & $33 \mathrm{~N}-10 \mathrm{~W}-01 \mathrm{ADC}$ & 1972 & 50 & 6,260 & Terrace deposits \\
\hline 18 & Steven McCullough & 370733107523801 & $33 \mathrm{~N}-10 \mathrm{~W}-12 \mathrm{AAA}$ & 1969 & 116 & 6,240 & Animas Fm \\
\hline 19 & Hinio Tucson & 370730107533601 & $33 \mathrm{~N}-10 \mathrm{~W}-12 \mathrm{BBB}$ & 1970 & 94 & 6,160 & Animas Fm \\
\hline 20 & Chuck Atwood & 370715107522201 & $33 \mathrm{~N}-09 \mathrm{~W}-07 \mathrm{BCA}$ & 1987 & 220 & 6,250 & Animas Fm \\
\hline 21 & Dorene Fickett & 370714107524701 & $33 \mathrm{~N}-10 \mathrm{~W}-12 \mathrm{ADB}$ & 1976 & 65 & 6,230 & Terrace deposits \\
\hline 22 & W.J. Hallock & 370655107523901 & $33 \mathrm{~N}-10 \mathrm{~W}-12 \mathrm{DAD}$ & 1984 & 140 & 6,210 & Terrace deposits \\
\hline 23 & Jack Benton & 370653107525501 & $33 \mathrm{~N}-10 \mathrm{~W}-12 \mathrm{DCA}$ & 1971 & 49 & 6,185 & Terrace deposits \\
\hline 24 & Skip White & 370650107522701 & $33 \mathrm{~N}-09 \mathrm{~W}-07 \mathrm{CCB}$ & 1982 & 40 & 6,230 & Animas Fm \\
\hline 25 & Rusty Bonsor & 370645107534301 & $33 \mathrm{~N}-10 \mathrm{~W}-11 \mathrm{DDD}$ & 1986 & 86 & 6,150 & Nacimiento Fm \\
\hline 26 & Katherine Dale & 370638107530501 & $33 \mathrm{~N}-10 \mathrm{~W}-13 \mathrm{BAA}$ & 1986 & 150 & 6,185 & Animas Fm \\
\hline 27 & Gerald Zink & 370637107525001 & $33 \mathrm{~N}-10 \mathrm{~W}-13 \mathrm{ABA}$ & $\sim 1935$ & 50 & 6,180 & Terrace deposits \\
\hline 28 & Gary Pasco & 370632107535101 & $33 \mathrm{~N}-10 \mathrm{~W}-14 \mathrm{AAC}$ & 1958 & 65 & 6,150 & Terrace deposits \\
\hline 29 & Neal Edwards & 370630107522901 & $33 \mathrm{~N}-09 \mathrm{~W}-18 \mathrm{BBC}$ & 1980 & 120 & 6,215 & Animas Fm \\
\hline 30 & Winona Morgan & 370627107525101 & $33 \mathrm{~N}-10 \mathrm{~W}-13 \mathrm{ACA}$ & 1946 & 63 & 6,185 & Animas Fm \\
\hline
\end{tabular}


Tabie 1. Records of sampled water wells and springs--Continued

\begin{tabular}{|c|c|c|c|c|c|c|c|}
\hline $\begin{array}{c}\text { Number } \\
\text { on } \\
\text { plate } 1\end{array}$ & $\begin{array}{c}\text { Well or spring } \\
\text { owner }\end{array}$ & Station number & Land-net location & $\begin{array}{l}\text { Year } \\
\text { drilled }\end{array}$ & $\begin{array}{c}\text { Total } \\
\text { depth } \\
\text { of } \\
\text { well }\end{array}$ & $\begin{array}{l}\text { Altitude } \\
\text { of land } \\
\text { surface }\end{array}$ & Aquifer \\
\hline 31 & Tony Schweikle & 370612107522201 & $33 \mathrm{~N}-09 \mathrm{~W}-18 \mathrm{CBA}$ & 1975 & 110 & 6,220 & Animas Fm \\
\hline 32 & Andy Chilton & 370603107522901 & $33 \mathrm{~N}-09 \mathrm{~W}-18 \mathrm{CBC}$ & 1982 & 130 & 6,175 & Animas Fm \\
\hline 33 & Don Carrol & 370602107524001 & $33 \mathrm{~N}-10 \mathrm{~W}-13 \mathrm{DAC}$ & 1974 & 52 & 6,175 & Terrace deposits \\
\hline 34 & Seven Mazzone & 370601107524801 & $33 N-10 W-13 D X$ & -- & 41 & 6,170 & Terrace deposits \\
\hline 35 & James Standifer & 370552107521801 & $33 N-09 W-18 C C D$ & 1985 & 140 & 6,190 & Animas Fm \\
\hline 36 & Terry Obery & 370548107524701 & $33 \mathrm{~N}-10 \mathrm{~W}-13 \mathrm{DDC}$ & 1985 & 150 & 6,170 & Animas Fm \\
\hline 37 & Carl Johnson & 370508107530701 & $33 \mathrm{~N}-10 \mathrm{~W}-24 \mathrm{CDA}$ & -- & 70 & 6,130 & Terrace deposits \\
\hline 38 & Karen Harrison & 370450107530701 & $33 \mathrm{~N}-10 \mathrm{~W}-25 \mathrm{BAA}$ & $\sim 1975$ & 55 & 6,130 & Terrace deposits \\
\hline 39 & Patty Haneman & 370443107531901 & $33 \mathrm{~N}-10 \mathrm{~W}-25 \mathrm{BAC}$ & 1982 & 230 & 6,150 & Nacimiento Fm \\
\hline 40 & Lawrence Craig & 370443107530301 & $33 \mathrm{~N}-10 \mathrm{~W}-25 \mathrm{ABC}$ & 1940 & 43 & 6,120 & Terrace deposits \\
\hline 41 & Joe Williams & 370442107525101 & $33 \mathrm{~N}-10 \mathrm{~W}-25 \mathrm{ACA}$ & 1976 & 60 & 6,110 & Terrace deposits \\
\hline 42 & Gerald Brown & 370437107530201 & $33 \mathrm{~N}-10 \mathrm{~W}-25 \mathrm{ACB}$ & 1950 & 130 & 6,110 & Nacimiento Fm \\
\hline 43 & John Gamble & 370437107524201 & $33 \mathrm{~N}-10 \mathrm{~W}-25 \mathrm{ADB}$ & 1986 & 260 & 6,090 & Nacimiento Fm \\
\hline 44 & Jerry Schane & 370430107531801 & $33 N-10 W-25 B D C$ & 1979 & 110 & 6,150 & Nacimiento Fm \\
\hline 45 & Neal Short & 370412107530701 & $33 \mathrm{~N}-10 \mathrm{~W}-25 \mathrm{CDA}$ & 1905 & 50 & 6,120 & Terrace deposits \\
\hline 46 & David Huffman & 370403107525201 & $33 \mathrm{~N}-10 \mathrm{~W}-25 \mathrm{DCD}$ & 1981 & 127 & 6,090 & Nacimiento Fm \\
\hline 47 & Randy McKee & 370359107530401 & $33 N-10 W-36 A B B$ & $\sim 1950$ & 72 & 6,120 & Nacimiento Fm \\
\hline 48 & Randy McKee & 370355107524101 & $33 \mathrm{~N}-10 \mathrm{~W}-36 \mathrm{AAC}$ & 1984 & 142 & 6,110 & Nacimiento Fm \\
\hline 49 & Edith Rhodes & 370347107525201 & $33 \mathrm{~N}-10 \mathrm{~W}-36 \mathrm{ACA}$ & - & 180 & 6,090 & Nacimiento Fm \\
\hline 50 & Lester Davis & 370347107520801 & $33 N-09 W-31 B D B$ & 1977 & 218 & 6,080 & Nacimiento Fm \\
\hline 51 & Doyle Hartman & 370343107521701 & $33 N-09 W-31 B D C$ & 1983 & 140 & 6,060 & Nacimiento Fm \\
\hline 52 & Jack Kloepfer & 370337107524901 & $33 N-10 W-36 A C D$ & $\sim 1950$ & 130 & 6,080 & Nacimiento Fm \\
\hline 53 & Carl Weston & 370337107522801 & $33 N-09 W-31 \mathrm{CBB}$ & 1986 & 217 & 6,060 & Nacimiento Fm \\
\hline 54 & Catherine Sutton & 370332107525301 & $33 \mathrm{~N}-10 \mathrm{~W}-36 \mathrm{DBA}$ & 1987 & 140 & 6,080 & Nacimiento Fm \\
\hline 55 & Catherine Sutton & 370332107525302 & $33 N-10 W-36 D B A$ & 1961 & 35 & 6,080 & Terrace deposits \\
\hline 56 & Rick Heinz & 370323107520901 & $33 \mathrm{~N}-09 \mathrm{~W}-31 \mathrm{CDB}$ & 1983 & 12 & 6,020 & Valley fill \\
\hline 57 & David Temple & 370319107525201 & $33 \mathrm{~N}-10 \mathrm{~W}-36 \mathrm{DCA}$ & 1980 & 199 & 6,070 & Nacimiento Fm \\
\hline 58 & Carl Weston & 370316107522701 & $33 \mathrm{~N}-09 \mathrm{~W}-31 \mathrm{CCC}$ & 1968 & 201 & 6,040 & Nacimiento Fm \\
\hline 59 & Maurice Walter & 370301107523701 & $32 \mathrm{~N}-10 \mathrm{~W}-01 \mathrm{AAD}$ & 1960 & 112 & 6,050 & Nacimiento Fm \\
\hline 60 & Trans Ohio Bank & 370257107523301 & $32 \mathrm{~N}-10 \mathrm{~W}-01 \mathrm{ADA}$ & 1975 & 128 & 6,040 & Nacimiento Fm \\
\hline
\end{tabular}


Table 1. Records of sampled water wells and springs--Continued

\begin{tabular}{|c|c|c|c|c|c|c|c|}
\hline $\begin{array}{c}\text { Number } \\
\text { on } \\
\text { plate } 1\end{array}$ & $\begin{array}{l}\text { Well or spring } \\
\text { owner }\end{array}$ & Station number & Land-net location & $\begin{array}{l}\text { Year } \\
\text { drilled }\end{array}$ & $\begin{array}{c}\text { Total } \\
\text { depth } \\
\text { of } \\
\text { well }\end{array}$ & $\begin{array}{l}\text { Altitude } \\
\text { of land } \\
\text { surface }\end{array}$ & Aquifer \\
\hline 61 & Junior Bonds & 370248107524501 & $32 \mathrm{~N}-10 \mathrm{~W}-01 \mathrm{ADC}$ & 1945 & 60 & 6,060 & Nacimiento Fm \\
\hline 62 & Bill Mullins & 370245107523001 & $32 \mathrm{~N}-09 \mathrm{~W}-06 \mathrm{CBC}$ & -- & 25 & 6,025 & Terrace deposits \\
\hline 63 & Junior Bonds & 370208107523301 & $32 \mathrm{~N}-10 \mathrm{~W}-12 \mathrm{AAD}$ & 1946 & 80 & 6,000 & Nacimiento Fm \\
\hline 64 & Jenny Boyer & 370133107524601 & $32 \mathrm{~N}-10 \mathrm{~W}-12 \mathrm{DDC}$ & 1960 & 121 & 6,080 & Nacimiento Fm \\
\hline 65 & Blanton Cogburn & 370127107522801 & $32 \mathrm{~N}-09 \mathrm{~W}-07 \mathrm{CCC}$ & 1987 & 140 & 5,980 & Nacimiento Fm \\
\hline 66 & John Goldman & 370117107522801 & $32 \mathrm{~N}-09 \mathrm{~W}-18 \mathrm{BBC}$ & -- & 120 & 5,985 & Nacimiento Fm \\
\hline 67 & Blanton Cogburn & 370117107522501 & $32 \mathrm{~N}-09 \mathrm{~W}-18 \mathrm{BBD}$ & 1973 & 50 & 5,970 & Terrace deposits \\
\hline 68 & Foy Cogburn & 370104107522701 & $32 \mathrm{~N}-09 \mathrm{~W}-18 \mathrm{BCC}$ & 1967 & 170 & 5,990 & Nacimiento Fm \\
\hline 69 & Foy Cogburn & 370103107522501 & $32 \mathrm{~N}-09 \mathrm{~W}-18 \mathrm{BCD}$ & 1966 & 60 & 5,970 & Nacimiento Fm \\
\hline 70 & Robert Kinslow & 370055107522801 & $32 \mathrm{~N}-09 \mathrm{~W}-18 \mathrm{CBB}$ & -- & 80 & 6,015 & Nacimiento Fm \\
\hline 71 & Richard Banes & 370054107522001 & $32 \mathrm{~N}-09 \mathrm{~W}-18 \mathrm{CBD}$ & 1962 & 14 & 5,950 & Valley fill \\
\hline 72 & L. Dean Johnson & 370003107520301 & $32 \mathrm{~N}-09 \mathrm{~W}-19 \mathrm{CAA}$ & -- & 58 & 5,930 & Valley fill \\
\hline 73 & Patricia Johnson & 365938107521701 & $32 \mathrm{~N}-10 \mathrm{~W}-10 \mathrm{CDD}$ & 1977 & 280 & 6,000 & Nacimiento Fm \\
\hline 74 & Donna Gilbert & 365911107522001 & $32 \mathrm{~N}-10 \mathrm{~W}-15 \mathrm{BDC}$ & -. & 116 & 5,970 & Nacimiento Fm \\
\hline 75 & R. Shindledecker & 365910107520701 & $32 \mathrm{~N}-10 \mathrm{~W}-15 \mathrm{ACC}$ & 1983 & 102 & 5,925 & Nacimiento Fm \\
\hline 76 & James Wilkerson & 365902107523301 & $32 \mathrm{~N}-10 \mathrm{~W}-15 \mathrm{CBA}$ & 1983 & 155 & 5,990 & Nacimiento Fm \\
\hline 77 & Leroy Bussel & 365855107522101 & $32 \mathrm{~N}-10 \mathrm{~W}-15 \mathrm{CAC}$ & -- & 44 & 5,940 & Terrace deposits \\
\hline 78 & Wesley Bond & 365838107523101 & $32 \mathrm{~N}-10 \mathrm{~W}-22 \mathrm{BBA}$ & 1943 & 70 & 5,940 & Nacimiento Fm \\
\hline 79 & Linn Blancett & 365838107520801 & $32 \mathrm{~N}-10 \mathrm{~W}-22 \mathrm{BAA}$ & 1976 & 99 & 5,930 & Nacimiento Fm \\
\hline 80 & Pat Knepp & 365818107525701 & $32 \mathrm{~N}-10 \mathrm{~W}-21 \mathrm{ACD}$ & 1982 & 105 & 5,960 & Nacimiento Fm \\
\hline 81 & James Welles & 365807107522701 & $32 \mathrm{~N}-10 \mathrm{~W}-22 \mathrm{CBD}$ & 1974 & 20 & 5,885 & Terrace deposits \\
\hline 82 & Fred Clark & 365803107524301 & $32 \mathrm{~N}-10 \mathrm{~W}-21 \mathrm{DAD}$ & 1967 & 104 & 5,910 & Nacimiento Fm \\
\hline 83 & Mitch Waggoner & 365758107525801 & $32 \mathrm{~N}-10 \mathrm{~W}-21 \mathrm{DCA}$ & 1981 & 70 & 5,950 & Nacimiento Fm \\
\hline 84 & Dwayne Lewis & 365752107530301 & $32 \mathrm{~N}-10 \mathrm{~W}-21 \mathrm{DCD}$ & 1980 & 255 & 6,060 & Nacimiento Fm \\
\hline 85 & James Musgrove ${ }^{1}$ & 365751107525201 & $32 \mathrm{~N}-10 \mathrm{~W}-21 \mathrm{DDC}$ & -- & 0 & 5,880 & Nacimiento Fm \\
\hline 86 & Ray Kysar & 365733107523801 & $32 \mathrm{~N}-10 \mathrm{~W}-27 \mathrm{BCB}$ & 1980 & 38 & 5,880 & Terrace deposits \\
\hline 87 & Ray Kysar & 365717107524101 & $32 \mathrm{~N}-10 \mathrm{~W}-28 \mathrm{DAA}$ & -- & 39 & 5,890 & Terrace deposits \\
\hline 88 & Ray Kysar & 365655107524601 & $32 \mathrm{~N}-10 \mathrm{~W}-33 \mathrm{AAA}$ & -- & 29 & 5,860 & Terrace deposits \\
\hline 89 & Lanier Clark & 365637107524501 & $32 \mathrm{~N}-10 \mathrm{~W}-33 \mathrm{ADD}$ & $\sim 1925$ & 50 & 5,860 & Valley fill \\
\hline 90 & Jay Riley & 365636107524801 & $32 \mathrm{~N}-10 \mathrm{~W}-33 \mathrm{ADD}$ & 1980 & 42 & 5,870 & Valley fill \\
\hline
\end{tabular}


Table 1. Records of sampled water wells and springs--Continued

\begin{tabular}{|c|c|c|c|c|c|c|c|}
\hline $\begin{array}{c}\text { Number } \\
\text { on } \\
\text { plate } 1\end{array}$ & $\begin{array}{c}\text { Well or spring } \\
\text { owner }\end{array}$ & Station number & Land-net location & $\begin{array}{l}\text { Year } \\
\text { drilled }\end{array}$ & $\begin{array}{c}\text { Total } \\
\text { depth } \\
\text { of } \\
\text { well }\end{array}$ & $\begin{array}{l}\text { Altitude } \\
\text { of land } \\
\text { surface }\end{array}$ & Aquifer \\
\hline$\overline{91}$ & Faye Warren & 365632107524801 & $32 \mathrm{~N}-10 \mathrm{~W}-33 \mathrm{ADD}$ & 1984 & 80 & 5,870 & Valley fill \\
\hline 92 & Stanley Lanier & 365632107524201 & $32 \mathrm{~N}-10 \mathrm{~W}-33 \mathrm{ADD}$ & 1976 & 49 & 5,850 & Valley fill \\
\hline 93 & Paul Rouse & 365626107523701 & $32 \mathrm{~N}-10 \mathrm{~W}-34 \mathrm{CBA}$ & $\sim 1900$ & 23 & 5,860 & Valley fill \\
\hline 94 & Clarence Hunter & 365625107531101 & $32 \mathrm{~N}-10 \mathrm{~W}-33 \mathrm{DBB}$ & $\sim 1950$ & 48 & 5,880 & Valley fill \\
\hline 95 & Bill Townsend & 365625107525201 & $32 \mathrm{~N}-10 \mathrm{~W}-33 \mathrm{DAB}$ & 1980 & 45 & 5,860 & Valley fill \\
\hline 96 & Maxine Welch & 365623107523301 & $32 \mathrm{~N}-10 \mathrm{~W}-34 \mathrm{CBD}$ & 1977 & 40 & 5,850 & Valley fill \\
\hline 97 & Thomas McCartney & 365622107530001 & $32 \mathrm{~N}-10 \mathrm{~W}-33 \mathrm{DBD}$ & 1987 & 76 & 5,880 & Valley fill \\
\hline 98 & Keith Benfield & 365621107532501 & $32 \mathrm{~N}-10 \mathrm{~W}-33 \mathrm{CAC}$ & 1982 & 75 & 5,880 & Valley fill \\
\hline 99 & Benson Leeper & 365619107533001 & $32 \mathrm{~N}-10 \mathrm{~W}-33 \mathrm{CBD}$ & 1980 & 70 & 5,880 & Valley fill \\
\hline 100 & Mary Brimhall & 365619107531101 & $32 \mathrm{~N}-10 \mathrm{~W}-33 \mathrm{DBC}$ & 1982 & 60 & 5,860 & Valley fill \\
\hline 101 & Jim Englert & 365618107530801 & $32 \mathrm{~N}-10 \mathrm{~W}-33 \mathrm{DBC}$ & -- & 60 & 5,860 & Valley fill \\
\hline 102 & Richard Lopez & 365618107523501 & $32 \mathrm{~N}-10 \mathrm{~W}-34 \mathrm{CBD}$ & 1979 & 31 & 5,840 & Valley fill \\
\hline 103 & Joe Anderson & 365617107525901 & $32 \mathrm{~N}-10 \mathrm{~W}-33 \mathrm{DCA}$ & 1965 & 33 & 5,855 & Valley fill \\
\hline 104 & Jimmy Reiter & 365617107523401 & $32 \mathrm{~N}-10 \mathrm{~W}-34 \mathrm{CCA}$ & 1987 & 28 & 5,850 & Valley fill \\
\hline 105 & Benson Leeper & 365616107533601 & $32 \mathrm{~N}-10 \mathrm{~W}-33 \mathrm{CCA}$ & 1984 & 60 & 5,870 & Valley fill \\
\hline 106 & Gene Brown & 365614107531301 & $32 \mathrm{~N}-10 \mathrm{~W}-33 \mathrm{CDA}$ & $\sim 1935$ & 36 & 5,865 & Valley fill \\
\hline 107 & Benson Leeper & 365613107534501 & $32 \mathrm{~N}-10 \mathrm{~W}-32 \mathrm{DDA}$ & 1946 & 40 & 5,860 & Valley fill \\
\hline 108 & Donald Martinez & 365613107523901 & $32 \mathrm{~N}-10 \mathrm{~W}-34 \mathrm{CCA}$ & $\sim 1920$ & 30 & 5,840 & Valley fill \\
\hline 109 & Robert McClanahan & 365613107523201 & $32 \mathrm{~N}-10 \mathrm{~W}-34 \mathrm{CCA}$ & -- & 15 & 5,850 & Valley fill \\
\hline 110 & Inez McCloud & 365612107532501 & $32 \mathrm{~N}-10 \mathrm{~W}-33 \mathrm{CDB}$ & 1935 & 35 & 5,850 & Valley fill \\
\hline 111 & Wilbur Brewer & 365610107531801 & $32 \mathrm{~N}-10 \mathrm{~W}-33 \mathrm{CDD}$ & 1978 & 62 & 5,860 & Valley fill \\
\hline 112 & Benson Leeper & 365609107534501 & $32 \mathrm{~N}-10 \mathrm{~W}-32 \mathrm{DDD}$ & 1950 & 40 & 5,850 & Valley fill \\
\hline 113 & George Betz & 365609107531101 & $32 \mathrm{~N}-10 \mathrm{~W}-33 \mathrm{DCC}$ & $\sim 1900$ & 60 & 5,860 & Valley fill \\
\hline 114 & Lee Flaherty & 365605107540001 & $32 \mathrm{~N}-10 \mathrm{~W}-32 \mathrm{DDC}$ & 1962 & 26 & 5,850 & Valley fill \\
\hline 115 & Henry Knowlton & 365604107542001 & $31 \mathrm{~N}-10 \mathrm{~W}-05 \mathrm{BAA}$ & 1975 & 14 & 5,810 & Valley fill \\
\hline 116 & Caleb Dickson & 365604107530101 & $31 \mathrm{~N}-10 \mathrm{~W}-04 \mathrm{ABA}$ & 1985 & 40 & 5,835 & Valley fill \\
\hline 117 & James Duke & 365604107523801 & $31 \mathrm{~N}-10 \mathrm{~W}-03 \mathrm{BBB}$ & 1981 & 35 & 5,835 & Valley fill \\
\hline 118 & Keith Rhodes & 365603107541101 & $31 \mathrm{~N}-10 \mathrm{~W}-05 \mathrm{ABB}$ & 1974 & 12 & 5,810 & Valley fill \\
\hline 119 & John Zoller & 365603107531001 & $31 \mathrm{~N}-10 \mathrm{~W}-04 \mathrm{ABB}$ & 1986 & 55 & 5,855 & Valley fill \\
\hline 120 & Charles Head & 365603107524601 & $31 \mathrm{~N}-10 \mathrm{~W}-04 \mathrm{AAA}$ & 1979 & 99 & 5,850 & Nacimiento Fm \\
\hline
\end{tabular}


Table 1. Records of sampled water wells and springs--Continued

\begin{tabular}{|c|c|c|c|c|c|c|c|}
\hline $\begin{array}{c}\text { Number } \\
\text { on } \\
\text { plate } 1\end{array}$ & $\begin{array}{l}\text { Well or spring } \\
\text { owner }\end{array}$ & Station number & Land-net location & $\begin{array}{l}\text { Year } \\
\text { drilled }\end{array}$ & $\begin{array}{l}\text { Total } \\
\text { depth } \\
\text { of } \\
\text { well }\end{array}$ & $\begin{array}{l}\text { Altitude } \\
\text { of land } \\
\text { surface }\end{array}$ & Aquifer \\
\hline 121 & Ken Stanley & 365602107533301 & $31 \mathrm{~N}-10 \mathrm{~W}-04 \mathrm{BBA}$ & $\sim 1950$ & 35 & 5,830 & Valley fill \\
\hline 122 & Bill Metz & 365558107525601 & $31 \mathrm{~N}-10 \mathrm{~W}-04 \mathrm{AAC}$ & 1976 & 66 & 5,850 & Valley fill \\
\hline 123 & Glen Rhodes & 365553107540001 & $31 \mathrm{~N}-10 \mathrm{~W}-05 \mathrm{AAC}$ & 1968 & 10 & 5,815 & Valley fill \\
\hline 124 & Marshall Johnson & 365553107532901 & $31 \mathrm{~N}-10 \mathrm{~W}-04 \mathrm{BBD}$ & 1978 & 26 & 5,820 & Valley fill \\
\hline 125 & Melvin Johnson & 365553107530401 & $31 \mathrm{~N}-10 \mathrm{~W}-04 \mathrm{ABD}$ & $\sim 1955$ & 40 & 5,840 & Valley fill \\
\hline 126 & Frank Nordstrom & 365552107531201 & $31 \mathrm{~N}-10 \mathrm{~W}-04 \mathrm{ACB}$ & 1977 & 28 & 5,830 & Valley fill \\
\hline 127 & $\begin{array}{l}\text { Dutchman Hills } \\
\text { Water Company }\end{array}$ & 365550107531701 & $31 \mathrm{~N}-10 \mathrm{~W}-04 \mathrm{BDA}$ & 1978 & 90 & 5,820 & Nacimiento Fm \\
\hline 128 & $\begin{array}{l}\text { Dutchman Hills } \\
\text { Water Company }\end{array}$ & 365549107531701 & $31 \mathrm{~N}-10 \mathrm{~W}-04 \mathrm{BDA}$ & 1978 & 70 & 5,820 & Nacimiento Fm \\
\hline 129 & Keith Englehart & 365548107543501 & $31 \mathrm{~N}-10 \mathrm{~W}-05 \mathrm{BCA}$ & 1985 & 60 & 5,810 & Valley fill \\
\hline 130 & Jerry Marcotte & 365548107535201 & $31 \mathrm{~N}-10 \mathrm{~W}-05 \mathrm{ADA}$ & -- & 30 & 5,825 & Valley fill \\
\hline 131 & Laverne Hill & 365545107532901 & $31 \mathrm{~N}-10 \mathrm{~W}-04 \mathrm{BCD}$ & $\sim 1960$ & 30 & 5,825 & Valley fill \\
\hline 132 & Glen Rhodes & 365538107540701 & $31 \mathrm{~N}-10 \mathrm{~W}-05 \mathrm{DBA}$ & 1963 & 45 & 5,820 & Valley fill \\
\hline 133 & Pat Cugnini & 365533107544601 & $31 \mathrm{~N}-10 \mathrm{~W}-05 \mathrm{CBC}$ & -- & 40 & 5,820 & Valley fill \\
\hline 134 & Helen Moss & 365531107535901 & $31 \mathrm{~N}-10 \mathrm{~W}-05 \mathrm{DAC}$ & 1971 & 42 & 5,830 & Valley fill \\
\hline 135 & Paul Kennedy & 365528107541301 & $31 \mathrm{~N}-10 \mathrm{~W}-05 \mathrm{DBC}$ & 1965 & 33 & 5,820 & Valley fill \\
\hline 136 & Bryan Burge & 365523107540301 & $31 \mathrm{~N}-10 \mathrm{~W}-05 \mathrm{DCA}$ & 1982 & 60 & 5,830 & Nacimiento Fm \\
\hline 137 & Jim Wilson & 365523107535301 & $31 \mathrm{~N}-10 \mathrm{~W}-05 \mathrm{DDB}$ & 1983 & 62 & 5,850 & Nacimiento Fm \\
\hline 138 & Ronald Osborn & 365522107543101 & $31 \mathrm{~N}-10 \mathrm{~W}-05 \mathrm{CDB}$ & 1980 & 4 & 5,800 & Valley fill \\
\hline 139 & Bud Beasley & 365521107541101 & $31 \mathrm{~N}-10 \mathrm{~W}-05 \mathrm{DCB}$ & 1982 & 40 & 5,820 & Valley fill \\
\hline 140 & Wright McEwen & 365518107542901 & $31 \mathrm{~N}-10 \mathrm{~W}-05 \mathrm{CDC}$ & 1990 & 35 & 5,815 & Valley fill \\
\hline 141 & Mike Carruthers & 365513107545401 & $31 \mathrm{~N}-10 \mathrm{~W}-07 \mathrm{AAA}$ & $\sim 1940$ & 20 & 5,795 & Valley fill \\
\hline 142 & Jess Satathite & 365513107541301 & $31 N-10 W-05 D C C$ & 1986 & 35 & 5,820 & Valley fill \\
\hline 143 & John Brandenburg & 365500107545401 & $31 \mathrm{~N}-10 \mathrm{~W}-07 \mathrm{AAD}$ & 1988 & 42 & 5,790 & Valley fill \\
\hline 144 & Enrique Del Vito & 365457107542801 & $31 \mathrm{~N}-10 \mathrm{~W}-08 \mathrm{BDB}$ & $\sim 1950$ & 25 & 5,810 & Valley fill \\
\hline 145 & Flora Griffin & 365450107542501 & $31 \mathrm{~N}-10 \mathrm{~W}-08 \mathrm{BDD}$ & $\sim 1950$ & 40 & 5,820 & Valley fill \\
\hline 146 & Lee Flaherty & 365439107545601 & $31 \mathrm{~N}-10 \mathrm{~W}-07 \mathrm{DAD}$ & 1987 & 30 & 5,780 & Valley fill \\
\hline 147 & C.A. Dickens & 365437107543801 & $31 \mathrm{~N}-10 \mathrm{~W}-08 \mathrm{CBD}$ & 1978 & -40 & 5,785 & Valley fill \\
\hline 148 & Odus Johns & 365433107550901 & $31 \mathrm{~N}-10 \mathrm{~W}-07 \mathrm{DCA}$ & 1983 & 35 & 5,785 & Valley fill \\
\hline 149 & Bill Greenhalgh & 365426107552501 & $31 \mathrm{~N}-10 \mathrm{~W}-07 \mathrm{CDD}$ & 1978 & 60 & 5,800 & Valley fill \\
\hline 150 & Willard Hottell & 365421107544301 & $31 \mathrm{~N}-10 \mathrm{~W}-08 \mathrm{CCD}$ & -- & 19 & 5,770 & Valley fill \\
\hline
\end{tabular}


Table 1. Records of sampled water wells and springs--Continued

\begin{tabular}{|c|c|c|c|c|c|c|c|}
\hline $\begin{array}{l}\text { Number } \\
\text { on } \\
\text { plate } 1\end{array}$ & $\begin{array}{l}\text { Well or spring } \\
\text { owner }\end{array}$ & Station number & Land-net location & $\begin{array}{c}\text { Year } \\
\text { drilled }\end{array}$ & $\begin{array}{l}\text { Total } \\
\text { depth } \\
\text { of } \\
\text { well }\end{array}$ & $\begin{array}{l}\text { Altitude } \\
\text { of land } \\
\text { surface }\end{array}$ & Aquifer \\
\hline 151 & Geraldine Powers & 365407107554201 & $31 \mathrm{~N}-10 \mathrm{~W}-18 \mathrm{BCA}$ & $\sim 1955$ & $\overline{32}$ & 5,870 & Valley fill \\
\hline 152 & Terry Kennedy & 3654031075555701 & $31 \mathrm{~N}-10 \mathrm{~W}-18 \mathrm{BCB}$ & 1980 & 35 & 5,790 & Valley fill \\
\hline 153 & David Tingstrom & 365400107561001 & $31 \mathrm{~N}-11 \mathrm{~W}-13 \mathrm{ADC}$ & 1982 & 60 & 5,780 & Valley fill \\
\hline 154 & Charles Martin & 365353107555201 & $31 \mathrm{~N}-10 \mathrm{~W}-18 \mathrm{CBB}$ & 1980 & 25 & 5,750 & Valley fill \\
\hline 155 & Len Chapman & 365352107553301 & $31 \mathrm{~N}-10 \mathrm{~W}-18 \mathrm{CAB}$ & 1985 & 20 & 5,740 & Valley fill \\
\hline 156 & Darwin Wilson & 365352107552901 & $31 \mathrm{~N}-10 \mathrm{~W}-18 \mathrm{CAA}$ & -- & 22 & 5,765 & Valley fill \\
\hline 157 & Lewis Oltmanns & 365347107562201 & $31 \mathrm{~N}-11 \mathrm{~W}-13 \mathrm{DBD}$ & 1976 & 18 & 5,730 & Valley fill \\
\hline 158 & Olan Pousson & 365343107554801 & $31 \mathrm{~N}-10 \mathrm{~W}-18 \mathrm{CBD}$ & 1981 & 26 & 5,740 & Valley fill \\
\hline 159 & Williard Gore & 365338107561701 & $31 \mathrm{~N}-11 \mathrm{~W}-13 \mathrm{DCA}$ & -. & 40 & 5,730 & Valley fill \\
\hline 160 & William Stallings & 365337107564301 & $31 \mathrm{~N}-11 \mathrm{~W}-13 \mathrm{CDB}$ & 1977 & 45 & 5,760 & Valley fill \\
\hline 161 & Ronald Custer & 365331107555801 & $31 \mathrm{~N}-11 \mathrm{~W}-13 \mathrm{DDD}$ & 1983 & 45 & 5,765 & Valley fill \\
\hline 162 & Don Norman & 365318107560001 & $31 \mathrm{~N}-11 \mathrm{~W}-24 \mathrm{AAD}$ & -- & 50 & 5,760 & Valley fill \\
\hline 163 & Bob Nyce & 365307107563501 & $31 \mathrm{~N}-11 \mathrm{~W}-24 \mathrm{BDD}$ & -- & 28 & 5,730 & Valley fill \\
\hline 164 & John Durham & 365307107560001 & $31 \mathrm{~N}-11 \mathrm{~W}-24 \mathrm{ADD}$ & 1981 & 92 & 5,780 & Valley fill \\
\hline 165 & Roger Bixler & 365303107561501 & $31 \mathrm{~N}-11 \mathrm{~W}-24 \mathrm{ACD}$ & 1969 & 30 & 5,750 & Valley fill \\
\hline 166 & Charles Turner & 365252107562701 & $31 \mathrm{~N}-11 \mathrm{~W}-24 \mathrm{DBC}$ & 1978 & 38 & 5,740 & Valley fill \\
\hline 167 & Leon Spiller & 365248107563001 & $31 \mathrm{~N}-11 \mathrm{~W}-24 \mathrm{CDA}$ & 1971 & 30 & 5,740 & Valley fill \\
\hline 168 & Leon Knowlton & 365242107574301 & $31 \mathrm{~N}-11 \mathrm{~W}-23 \mathrm{CDD}$ & 1988 & 40 & 5,720 & Valley fill \\
\hline 169 & Stan Maynes & 365242107571001 & $31 \mathrm{~N}-11 \mathrm{~W}-23 \mathrm{DDD}$ & 1975 & 30 & 5,715 & Valley fill \\
\hline 170 & S.E. Winters & 365240107561601 & $31 \mathrm{~N}-11 \mathrm{~W}-24 \mathrm{DCD}$ & 1981 & 101 & 5,790 & Valley fill \\
\hline 171 & Irvin Randlemon & 365238107564501 & $31 \mathrm{~N}-11 \mathrm{~W}-24 \mathrm{CDC}$ & 1987 & 47 & 5,730 & Valley fill \\
\hline 172 & Charles Randlemon & 365223107565701 & $31 \mathrm{~N}-11 \mathrm{~W}-25 \mathrm{BCB}$ & 1967 & 65 & 5,750 & Valley fill \\
\hline 173 & James Hall & 365212107575001 & $31 \mathrm{~N}-11 \mathrm{~W}-26 \mathrm{BDC}$ & 1980 & 49 & 5,795 & Valley fill \\
\hline 174 & M. Shackleford & 365210107570101 & $31 \mathrm{~N}-11 \mathrm{~W}-25 \mathrm{CBB}$ & 1957 & 90 & 5,755 & Valley fill \\
\hline 175 & James Almond & 365205107575301 & $31 \mathrm{~N}-11 \mathrm{~W}-26 \mathrm{CBA}$ & 1978 & 18 & 5,685 & Valley fill \\
\hline 176 & Mozelle Mickey & 365203107582701 & $31 \mathrm{~N}-11 \mathrm{~W}-27 \mathrm{DBD}$ & -- & 125 & 5,725 & Valley fill \\
\hline 177 & Carlos Marquez & 365202107570501 & $31 \mathrm{~N}-11 \mathrm{~W}-26 \mathrm{DAD}$ & 1981 & 69 & 5,760 & Valley fill \\
\hline 178 & Charles McFarland & 365200107572501 & $31 N-11 W-26 \mathrm{DBD}$ & 1947 & 42 & 5,730 & Valley fill \\
\hline 179 & Theron Whipple & 365P58107571101 & $31 \mathrm{~N}-11 \mathrm{~W}-26 \mathrm{DAC}$ & $\sim 1975$ & 90 & 5,750 & Valley fill \\
\hline 180 & Everett Lee & 365154107581801 & $31 \mathrm{~N}-11 \mathrm{~W}-27 \mathrm{DDB}$ & -- & 35 & 5,670 & Valley fill \\
\hline
\end{tabular}


Table 1. Records of sampled water wells and springs--Continued

\begin{tabular}{|c|c|c|c|c|c|c|c|}
\hline $\begin{array}{c}\text { Number } \\
\text { on } \\
\text { plate } 1\end{array}$ & $\begin{array}{l}\text { Well or spring } \\
\text { owner }\end{array}$ & Station number & Land-net location & $\begin{array}{l}\text { Year } \\
\text { drilled }\end{array}$ & $\begin{array}{c}\text { Total } \\
\text { depth } \\
\text { of } \\
\text { well }\end{array}$ & $\begin{array}{l}\text { Altitude } \\
\text { of land } \\
\text { surface }\end{array}$ & Aquifer \\
\hline 181 & C.E. Awtrey & 365152107583201 & $31 \mathrm{~N}-11 \mathrm{~W}-27 \mathrm{DCA}$ & 1988 & 54 & 5,680 & Valley fill \\
\hline 182 & C.E. Awtrey & 365147107583501 & $31 \mathrm{~N}-11 \mathrm{~W}-27 \mathrm{DCC}$ & -- & 47 & 5,690 & Valley fill \\
\hline 183 & Jim Houston & 365143107575501 & $31 \mathrm{~N}-11 \mathrm{~W}-35 \mathrm{BBA}$ & 1982 & 23 & 5,675 & Valley fill \\
\hline 184 & Lois Scott & 365137107581801 & $31 \mathrm{~N}-11 \mathrm{~W}-34 \mathrm{AAC}$ & -- & 22 & 5,665 & Valley fill \\
\hline 185 & Ernest Phelps & 365136107583301 & $31 \mathrm{~N}-11 \mathrm{~W}-34 \mathrm{ABC}$ & 1978 & 25 & 5,665 & Valley fill \\
\hline 186 & Dwayne Lillywhite & 365130107585101 & $31 \mathrm{~N}-11 \mathrm{~W}-34 \mathrm{BDB}$ & 1979 & 79 & 5,700 & Valley fill \\
\hline 187 & Emma Giles & 365128107572301 & $31 \mathrm{~N}-11 \mathrm{~W}-35 \mathrm{ACA}$ & 1980 & 100 & 5,740 & Valley fill \\
\hline 188 & Bruce Krueger & 365127107581901 & $31 \mathrm{~N}-11 \mathrm{~W}-34 \mathrm{ADB}$ & 1982 & 22 & 5,665 & Valley fill \\
\hline 189 & Lawton Williams & 365117107585501 & $31 \mathrm{~N}-11 \mathrm{~W}-34 \mathrm{CAB}$ & -- & 30 & 5,665 & Valley fill \\
\hline 190 & Don Taylor & 365116107581501 & $31 \mathrm{~N}-11 \mathrm{~W}-34 \mathrm{DAB}$ & 1983 & 10 & 5,665 & Valley fill \\
\hline 191 & Stanley Campbell & 365113107575301 & $31 \mathrm{~N}-11 \mathrm{~W}-35 \mathrm{CBA}$ & 1972 & 90 & 5,735 & Nacimiento Fm \\
\hline 192 & Houston Perry & 365103107581501 & $31 N-11 W-34 D D A$ & 1957 & 42 & 5,695 & Valley fill \\
\hline 193 & Carl Sexton & 365057107583901 & $31 \mathrm{~N}-11 \mathrm{~W}-34 \mathrm{DCC}$ & 1982 & 34 & 5,670 & Valley fill \\
\hline 194 & Don Taylor & 365052107582501 & $30 \mathrm{~N}-11 \mathrm{~W}-03 \mathrm{ABA}$ & 1970 & 40 & 5,660 & Valley fill \\
\hline 195 & Dewey Sexton & 365045107584701 & $30 \mathrm{~N}-11 \mathrm{~W}-03 \mathrm{BAD}$ & 1980 & 36 & 5,665 & Valley fill \\
\hline 196 & All Miller & 365038107590201 & $30 \mathrm{~N}-11 \mathrm{~W}-03 \mathrm{BCA}$ & 1988 & 25 & 5,640 & Valley fill \\
\hline 197 & K.C. Longwell & 365035107584001 & $30 \mathrm{~N}-11 \mathrm{~W}-03 \mathrm{ACB}$ & $\sim 1970$ & 21 & 5,660 & Valley fill \\
\hline 198 & Andres Martinez & 365029107591201 & $30 \mathrm{~N}-11 \mathrm{~W}-03 \mathrm{BCC}$ & 1966 & 32 & 5,630 & Valley fill \\
\hline 199 & Buster Jaquez & 365028107591301 & $30 \mathrm{~N}-11 \mathrm{~W}-04 \mathrm{DAA}$ & 1981 & 14 & 5,630 & Valley fill \\
\hline 200 & Joe Gomez & 365024107593201 & $30 \mathrm{~N}-11 \mathrm{~W}-04 \mathrm{DBA}$ & $\sim 1965$ & 40 & 5,640 & Valley fill \\
\hline 201 & Jeff Houser & 365020107584501 & $30 \mathrm{~N}-11 \mathrm{~W}-03 \mathrm{CAD}$ & 1984 & 36 & 5,660 & Valley fill \\
\hline 202 & Buster Jaquez & 365016107590401 & $30 \mathrm{~N}-11 \mathrm{~W}-03 \mathrm{CBD}$ & 1981 & 28 & 5,640 & Valley fill \\
\hline 203 & Kathy Daddow & 365014107592801 & $30 \mathrm{~N}-11 \mathrm{~W}-04 \mathrm{DDB}$ & -- & 7 & 5,610 & Valley fill \\
\hline 204 & Howard Stinson & 365004107591501 & $30 \mathrm{~N}-11 \mathrm{~W}-04 \mathrm{DDD}$ & 1978 & 30 & 5,645 & Valley fill \\
\hline 205 & Ken Shultz & 364942107590201 & $30 \mathrm{~N}-11 \mathrm{~W}-10 \mathrm{BCD}$ & 1977 & 72 & 5,670 & Valley fill \\
\hline
\end{tabular}

'Spring owner. 
Table 2. Water-level and water-quality measurements and nearby soil-gas-methane concentrations for selected water wells and springs

[Water levels are in feet below land surface; $\mu \mathrm{S} / \mathrm{cm}$, microsiemens per centimeter at 25 degrees Celsius; ${ }^{\circ} \mathrm{C}$, degrees Celsius; IT, incremental titration; mg/L, milligrams per liter; $\mathrm{mg} / \mathrm{L}_{\mathrm{g}}$, milligrams per liter of gas; $<$, less than; $\mu \mathrm{g} / \mathrm{L}$, micrograms per liter; --, no data]

\begin{tabular}{|c|c|c|c|c|c|c|c|c|c|c|}
\hline $\begin{array}{c}\text { Num- } \\
\text { ber } \\
\text { on } \\
\text { plate } \\
1\end{array}$ & $\begin{array}{c}\text { Date } \\
\text { sampled }\end{array}$ & $\begin{array}{l}\text { Water } \\
\text { level }\end{array}$ & $\begin{array}{c}\text { Specific } \\
\text { con- } \\
\text { ductance } \\
(\mu \mathrm{S} / \mathrm{cm})\end{array}$ & $\begin{array}{c}\text { pH } \\
\text { (standard } \\
\text { units) }\end{array}$ & $\begin{array}{l}\text { Temper- } \\
\text { ature, } \\
\text { water } \\
\left({ }^{\circ} \mathrm{C}\right)\end{array}$ & $\begin{array}{l}\text { Alkalinity } \\
\text { dis- } \\
\text { solved, } \\
\text { onsite, } \\
\text { IT } \\
\left(\mathrm{mg}^{2} \mathrm{~L} \text { as }\right. \\
\left.\mathrm{CaCO}_{3}\right)\end{array}$ & $\begin{array}{l}\text { Dis- } \\
\text { solved } \\
\text { solids, } \\
\text { sum of } \\
\text { constit- } \\
\text { uents } \\
\text { (mg/L) }\end{array}$ & $\begin{array}{c}\text { Calcium, } \\
\text { dlssolved } \\
\text { (mg/L as } \\
\text { Ca) }\end{array}$ & $\begin{array}{l}\text { Magne- } \\
\text { sium, } \\
\text { dissolved } \\
\text { (mg/L as } \\
\mathrm{Mg} \text { ) }\end{array}$ & $\begin{array}{c}\text { Sodium, } \\
\text { dissolved } \\
\text { (mg/L as } \\
\mathrm{Na})\end{array}$ \\
\hline 1 & $08-28-90$ & 14.3 & 1,000 & 7.3 & 12.0 & -- & -- & -- & - & -- \\
\hline 2 & $08-28-90$ & 62.5 & 730 & 7.9 . & 17.0 & 182 & 517 & 41 & 5.4 & 140 \\
\hline 3 & $08-27-90$ & 87.0 & 820 & 8.6 & 16.0 & -- & -- & -- & -- & -- \\
\hline 4 & $08-27-90$ & 170.1 & 540 & 8.9 & 16.0 & 179 & 300 & 2.4 & $<.01$ & 120 \\
\hline 5 & $10-19-90$ & 142.1 & 519 & 8.9 & 15.0 & -- & -- & -- & -- & -- \\
\hline 6 & 09-05-90 & 76.1 & 769 & 8.0 & 16.5 & -- & -- & -- & -- & -- \\
\hline 7 & $08-28-90$ & 140.7 & 600 & 8.9 & 17.0 & 144 & 335 & 3.1 & $<.01$ & 130 \\
\hline 8 & $08-27-90$ & - & 655 & 8.5 & 14.0 & -- & -- & - & -- & -- \\
\hline 9 & $08-29-90$ & -- & 623 & 8.8 & 14.0 & 216 & 391 & 3.7 & .23 & 140 \\
\hline 10 & $08-27-90$ & 64.7 & 760 & 8.5 & 12.0 & 196 & 436 & 13 & .88 & 160 \\
\hline 11 & $08-29-90$ & 10.2 & 788 & 8.0 & 13.0 & -- & -- & - & -- & -- \\
\hline 12 & $08-24-90$ & 32.2 & 832 & 8.3 & 12.5 & -- & -- & - & -- & -- \\
\hline 13 & $08-24-90$ & 15.8 & 825 & 7.0 & 13.5 & 356 & 482 & 92 & 17 & 63 \\
\hline 14 & $08-21-90$ & 47.3 & 880 & 8.5 & 13.0 & 277 & 502 & 5.0 & .13 & 190 \\
\hline 15 & $11-19-90$ & 14.9 & 786 & 7.5 & 12.0 & 230 & -- & -- & -- & - \\
\hline 16 & $08-24-90$ & - & 680 & 7.1 & 11.0 & 318 & 387 & 96 & 16 & 27 \\
\hline 17 & $08-24-90$ & 37.4 & 869 & 7.2 & 12.0 & - & -- & -- & -- & -- \\
\hline 18 & $08-24-90$ & 22.5 & 785 & 7.2 & 13.0 & -- & -- & - & -- & -- \\
\hline 19 & $08-21-90$ & 23.2 & 1,520 & 7.9 & 13.5 & -- & -- & -- & -- & -- \\
\hline 20 & $08-23-90$ & 50.4 & 888 & 7.5 & 12.5 & 337 & 507 & 42 & 8.9 & 140 \\
\hline 21 & $08-23-90$ & 33.8 & 690 & 7.3 & 11.5 & -- & -- & -- & -- & -- \\
\hline 22 & $08-23-90$ & 29.8 & 703 & 8.7 & 13.0 & - & -- & -- & - & -- \\
\hline 23 & $08-23-90$ & 18.3 & 732 & 7.2 & 15.0 & - & - & - & -- & -- \\
\hline 24 & $08-22-90$ & -- & 737 & 7.2 & 14.0 & -- & - & - & -- & -- \\
\hline 25 & $08-21-90$ & 53.0 & 980 & 8.2 & 13.0 & 290 & 579 & 6.7 & .22 & 220 \\
\hline 26 & $08-23-90$ & 13.4 & 728 & 7.7 & 13.0 & 253 & 424 & 39 & 9.0 & 110 \\
\hline 27 & $08-22-90$ & -- & 762 & 7.2 & 12.0 & -- & -- & -- & -- & .- \\
\hline 28 & $08-20-90$ & 48.2 & 1,010 & 7.6 & 12.5 & - & -- & -- & -- & -- \\
\hline 29 & $08-22-90$ & 47.5 & 810 & 7.6 & 13.0 & 267 & 509 & 23 & 4.4 & 160 \\
\hline 30 & $08-22-90$ & 20.5 & 710 & 7.4 & 17.0 & -- & -- & -- & -- & -- \\
\hline
\end{tabular}


Table 2. Water-level and water-quality measurements and nearby soil-gas-methane concentrations for selected water wells and springs--Continued

\begin{tabular}{|c|c|c|c|c|c|c|c|c|c|c|}
\hline $\begin{array}{l}\text { Num- } \\
\text { ber } \\
\text { on } \\
\text { plate } \\
1\end{array}$ & $\begin{array}{c}\text { Date } \\
\text { sampled }\end{array}$ & $\begin{array}{c}\text { Water } \\
\text { levei }\end{array}$ & $\begin{array}{c}\text { Specific } \\
\text { con- } \\
\text { ductance } \\
(\mu \mathrm{S} / \mathrm{cm})\end{array}$ & $\begin{array}{c}\text { pH } \\
\text { (standard } \\
\text { units) }\end{array}$ & $\begin{array}{c}\text { Temper- } \\
\text { ature, } \\
\text { water } \\
\left({ }^{\circ} \mathrm{C}\right)\end{array}$ & $\begin{array}{c}\text { Alkalinity } \\
\text { dis- } \\
\text { soived, } \\
\text { onsite, } \\
\text { IT } \\
\text { (mg/L as } \\
\left.\mathrm{CaCO}_{3}\right)\end{array}$ & $\begin{array}{l}\text { Dis- } \\
\text { soived } \\
\text { soilds, } \\
\text { sum of } \\
\text { constit- } \\
\text { uents } \\
\text { (mg/L) }\end{array}$ & $\begin{array}{c}\text { Calcium, } \\
\text { dissolved } \\
\text { (mg/L as } \\
\text { Ca) }\end{array}$ & $\begin{array}{l}\text { Magne- } \\
\text { sium, } \\
\text { dissoived } \\
\text { (mg/L as } \\
\mathbf{M g})\end{array}$ & $\begin{array}{c}\text { Sodium, } \\
\text { dissolved } \\
\text { (mg/l as } \\
\mathrm{Na})\end{array}$ \\
\hline 31 & $08-22-90$ & $=$ & 665 & 8.0 & 17.0 & - & -- & -- & - & $=$ \\
\hline 32 & $08-21-90$ & 22.0 & 803 & 7.5 & 12.0 & -- & -- & -- & -- & - \\
\hline 33 & $08-23-90$ & 9.5 & 829 & 7.1 & 13.0 & -- & -- & -- & -- & - \\
\hline 34 & $08-23-90$ & 17.3 & 774 & 7.1 & 13.0 & -- & -- & -- & -- & -- \\
\hline 35 & $08-22-90$ & 30.7 & 850 & 7.3 & 14.0 & 316 & 516 & 78 & 17 & 93 \\
\hline \multirow[t]{3}{*}{36} & $08-20-90$ & 21.9 & 710 & 7.6 & 12.5 & 257 & 414 & 39 & 6.0 & 110 \\
\hline & $11-15-90$ & 26.9 & 644 & 7.7 & 12.0 & -- & - & - & -- & -- \\
\hline & $03-06-91$ & 27.2 & 740 & 7.1 & 12.0 & - & -- & -- & -- & -- \\
\hline 37 & $08-16-90$ & 35.5 & 852 & 7.3 & 15.0 & -- & -- & -- & - & -- \\
\hline 38 & $08-15-90$ & 33.3 & 572 & 7.5 & 12.5 & 187 & 334 & 58 & 14 & 40 \\
\hline 39 & $08-15-90$ & 52.6 & 1,210 & 8.2 & 14.0 & 174 & 676 & 11 & .15 & 240 \\
\hline 40 & $08-15-90$ & 24.9 & 918 & 7.3 & 12.0 & - & -- & - & - & -- \\
\hline 41 & $08-17-90$ & 11.3 & 952 & 7.1 & 11.0 & 382 & 570 & 100 & 37 & 61 \\
\hline 42 & $08-17-90$ & -- & 1,130 & 7.7 & 13.0 & 288 & 814 & 32 & 1.3 & 250 \\
\hline 43 & $08-16-90$ & -- & 1,260 & 7.4 & 12.0 & 405 & 719 & 48 & 5.9 & 220 \\
\hline 44 & $08-14-90$ & 62.8 & 919 & 7.8 & 15.0 & -- & -- & - & -- & - \\
\hline 45 & $08-14-90$ & 23.6 & 667 & 7.4 & 13.0 & - & - & - & -- & - \\
\hline 46 & $08-16-90$ & - & 802 & 7.7 & 12.0 & 217 & 474 & 16 & 1.4 & 160 \\
\hline 47 & $08-14-90$ & - & 547 & 7.5 & 13.5 & -- & -- & -- & - & - \\
\hline 48 & $08-13-90$ & -- & 690 & 7.9 & 13.0 & -- & - & -- & -- & -- \\
\hline 49 & $08-10-90$ & 15.9 & 564 & 7.6 & 12.0 & 188 & 328 & 62 & 8.1 & 45 \\
\hline 50 & $08-08-90$ & 49.0 & 893 & 8.1 & 14.0 & 213 & 506 & 17 & .33 & 170 \\
\hline 51 & $08-17-90$ & - & 1,950 & 8.0 & 13.0 & - & - & - & - & - \\
\hline 52 & $08-09-90$ & 19.6 & 758 & 8.4 & 12.5 & 171 & 423 & 13 & .21 & 140 \\
\hline 53 & $08-07-90$ & 43.0 & 1,190 & 7.9 & 13.0 & 260 & 760 & 21 & .45 & 250 \\
\hline 54 & $10-29-90$ & 39.8 & 795 & 7.7 & 12.0 & 243 & 507 & 51 & 10 & 120 \\
\hline \multirow[t]{2}{*}{55} & $10-29-90$ & 10.8 & 780 & 7.2 & 13.5 & 272 & 493 & 100 & 23 & 36 \\
\hline & & & \multicolumn{8}{|c|}{, } \\
\hline 56 & $08-09-90$ & 7.9 & 622 & 7.1 & 14.0 & 264 & -- & -- & -- & -- \\
\hline 57 & $10-10-90$ & 56.8 & 720 & 7.8 & 13.0 & 237 & 481 & 41 & 4.2 & 130 \\
\hline 58 & $08-06-90$ & 46.6 & 1,780 & 7.6 & 12.5 & 282 & 916 & 60 & 9.4 & 270 \\
\hline 59 & $10-25-90$ & 16.3 & 900 & 7.8 & 12.0 & 251 & 524 & 43 & 4.4 & 140 \\
\hline 60 & $08-20-90$ & 11.9 & 898 & 7.3 & 13.0 & - & - & -- & -- & -- \\
\hline
\end{tabular}


Table 2. Water-level and water-quality measurements and nearby soil-gas-methane concentrations for selected water wells and springs--Continued

\begin{tabular}{|c|c|c|c|c|c|c|c|c|c|c|}
\hline $\begin{array}{c}\text { Num- } \\
\text { ber } \\
\text { on } \\
\text { plate } \\
1\end{array}$ & $\begin{array}{l}\text { Date } \\
\text { sampled }\end{array}$ & $\begin{array}{l}\text { Water } \\
\text { level }\end{array}$ & $\begin{array}{c}\text { Speclfic } \\
\text { con- } \\
\text { ductance } \\
(\mu \mathrm{S} / \mathrm{cm})\end{array}$ & $\begin{array}{c}\text { pH } \\
\text { (standard } \\
\text { units) }\end{array}$ & $\begin{array}{c}\text { Temper- } \\
\text { ature, } \\
\text { water } \\
\left({ }^{\circ} \mathrm{C}\right)\end{array}$ & 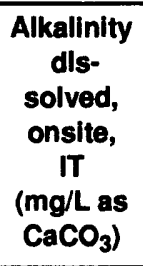 & $\begin{array}{c}\text { Dis- } \\
\text { solved } \\
\text { solids, } \\
\text { sum of } \\
\text { constit- } \\
\text { uents } \\
\text { (mg/L) }\end{array}$ & $\begin{array}{l}\text { Calclum, } \\
\text { dissolved } \\
\text { (mg/L as } \\
\text { Ca) }\end{array}$ & $\begin{array}{l}\text { Magne- } \\
\text { sium, } \\
\text { dissolved } \\
\text { (mg/L as } \\
\mathrm{Mg})\end{array}$ & $\begin{array}{c}\text { Sodium, } \\
\text { dissolved } \\
\text { (mg/L as } \\
\mathrm{Na})\end{array}$ \\
\hline 61 & $08-14-90$ & - & 769 & 7.3 & 15.5 & 276 & 478 & 110 & 21 & 29 \\
\hline 62 & $08-29-90$ & 11.3 & 888 & 7.1 & 16.0 & - & - & -- & -- & -- \\
\hline 63 & $08-13-90$ & -- & 1,460 & 7.1 & 11.5 & 408 & 924 & 110 & 35 & 160 \\
\hline 64 & $08-29-90$ & 72.9 & 2,880 & 7.2 & 14.5 & 275 & 2,040 & 190 & 42 & 420 \\
\hline 65 & $08-30-90$ & 21.6 & 3,250 & 7.9 & 14.0 & 225 & 2,230 & 110 & 8.9 & 730 \\
\hline 66 & $09-04-90$ & 33.4 & 1,410 & 8.3 & 13.0 & -- & -- & -- & - & -- \\
\hline 67 & $08-30-90$ & -- & 960 & 8.5 & 12.0 & -- & -- & -- & -- & -- \\
\hline 68 & $08-30-90$ & -- & 4,800 & 6.9 & 12.0 & -- & -- & -- & -- & -- \\
\hline 69 & $08-30-90$ & 13.7 & 2,770 & 7.2 & 16.0 & -- & -- & -- & -- & -- \\
\hline 70 & $08-30-90$ & -- & 1,210 & 8.4 & 20.0 & 198 & 668 & 8.8 & .6 & 240 \\
\hline 71 & $08-29-90$ & 3.2 & 604 & 7.4 & 18.0 & -- & -- & -- & -- & -- \\
\hline 72 & $09-10-90$ & 6.1 & 705 & 7.4 & 15.0 & -- & -- & -- & -- & -- \\
\hline 73 & $09-04-90$ & - & 4,050 & 8.1 & 15.0 & 77 & 2,040 & 45 & .72 & 730 \\
\hline 74 & $09-05-90$ & -- & 773 & 7.5 & 13.5 & -- & -- & -- & -- & -- \\
\hline 75 & $09-05-90$ & 15.9 & 757 & 7.2 & 15.0 & -- & -- & -- & -- & - \\
\hline 76 & $09-10-90$ & 68.1 & 2,790 & 7.5 & 14.0 & 501 & 1,670 & 40 & 18 & 550 \\
\hline 77 & $09-05-90$ & 22.8 & 740 & 7.2 & 16.0 & 252 & 432 & 110 & 18 & 19 \\
\hline 78 & $09-11-90$ & -- & 845 & 7.1 & 13.0 & -- & -- & -- & -- & -- \\
\hline 79 & $09-10-90$ & -- & 650 & 7.3 & 14.0 & -- & -- & -- & -- & -- \\
\hline 80 & $09-06-90$ & 59.7 & 3,300 & 7.2 & 15.5 & - & -- & - & -- & -- \\
\hline 81 & $09-07-90$ & 2.5 & 1,330 & 7.1 & 17.0 & -- & -- & -- & -- & -- \\
\hline 82 & $09-06-90$ & 19.5 & 1,240 & 8.1 & 13.5 & 234 & 727 & 22 & 3.5 & 240 \\
\hline 83 & $09-19-90$ & 37.4 & 1,050 & 7.4 & 14.5 & -- & - & -- & -- & -- \\
\hline 84 & $09-11-90$ & 94.7 & 5,200 & 7.3 & 15.5 & 323 & 2,970 & 150 & 19 & 890 \\
\hline 85 & $09-11-90$ & - & 775 & 7.3 & 14.0 & -- & - & -- & -- & -- \\
\hline 86 & $09-06-90$ & 10.0 & 959 & 7.1 & 14.5 & -- & -- & -- & -- & -- \\
\hline 87 & $09-07-90$ & 22.9 & 900 & 7.1 & 14.0 & 345 & 525 & 120 & 29 & 25 \\
\hline 88 & 09-07-90 & 15.0 & 1,080 & 7.2 & 14.0 & -- & -- & -- & -- & -- \\
\hline \multirow[t]{2}{*}{89} & $09-12-90$ & 22.0 & 1,130 & 7.1 & 14.5 & -- & -- & -- & -- & -- \\
\hline & $02-20-91$ & 26.0 & 1,235 & 6.8 & 13.5 & -- & -- & -- & -- & -- \\
\hline \multirow[t]{2}{*}{90} & $09-14-90$ & 28.5 & 774 & 7.2 & 15.5 & 270 & 464 & 100 & 23 & 31 \\
\hline & $02-22-91$ & 32.8 & 1,060 & 6.9 & 13.0 & -. & -- & -- & -- & -- \\
\hline
\end{tabular}


Table 2. Water-level and water-quality measurements and nearby soil-gas-methane concentrations for selected water wells and springs--Continued

\begin{tabular}{|c|c|c|c|c|c|c|c|c|c|c|}
\hline $\begin{array}{c}\text { Num- } \\
\text { ber } \\
\text { on } \\
\text { piate } \\
1\end{array}$ & $\begin{array}{c}\text { Date } \\
\text { sampied }\end{array}$ & $\begin{array}{c}\text { Water } \\
\text { level }\end{array}$ & $\begin{array}{c}\text { Specific } \\
\text { con- } \\
\text { ductance } \\
(\mu \mathrm{S} / \mathrm{cm})\end{array}$ & $\begin{array}{c}\text { pH } \\
\text { (standard } \\
\text { units) }\end{array}$ & $\begin{array}{c}\text { Temper- } \\
\text { ature, } \\
\text { water } \\
\left({ }^{\circ} \mathrm{C}\right)\end{array}$ & $\begin{array}{l}\text { Alkaiinity } \\
\text { dis- } \\
\text { soived, } \\
\text { onsite, } \\
\text { IT } \\
(\mathrm{mg} / \mathrm{L} \text { as } \\
\left.\mathrm{CaCO}_{3}\right)\end{array}$ & $\begin{array}{c}\text { Dis- } \\
\text { soived } \\
\text { soilds, } \\
\text { sum of } \\
\text { constit- } \\
\text { uents } \\
\text { (mg/L) }\end{array}$ & $\begin{array}{c}\text { Caicium, } \\
\text { dissoived } \\
\text { (mg/L as } \\
\text { Ca) }\end{array}$ & $\begin{array}{l}\text { Magne- } \\
\text { sium, } \\
\text { dissoived } \\
\text { (mg/L as } \\
\mathrm{Mg})\end{array}$ & $\begin{array}{c}\text { Sodium, } \\
\text { dissoived } \\
\text { (mg/L as } \\
\mathrm{Na} \text { ) }\end{array}$ \\
\hline 91 & $09-13-90$ & 25.6 & 649 & 6.9 & 15.5 & -- & -- & -- & -- & 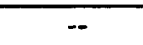 \\
\hline \multirow[t]{2}{*}{92} & $09-13-90$ & -- & 1,200 & 7.0 & 16.5 & 351 & 680 & 130 & 30 & 69 \\
\hline & $02-21-91$ & -- & 1,630 & 6.9 & 11.0 & -- & -- & -- & -- & - \\
\hline \multirow[t]{2}{*}{93} & $09-13-90$ & -- & 660 & 7.4 & 17.0 & -- & -- & -- & -- & -- \\
\hline & $02-19-91$ & -- & 960 & 6.9 & 11.5 & -- & -- & -- & -- & -- \\
\hline \multirow[t]{2}{*}{94} & $09-12-90$ & 37.3 & 610 & 7.4 & 14.5 & -- & -- & -- & -- & -- \\
\hline & $02-25-91$ & 49.5 & 730 & 6.9 & 13.5 & -- & -- & -- & -- & -- \\
\hline 95 & $09-19-90$ & 23.0 & 680 & 7.4 & 15.0 & - & -- & -- & -- & -- \\
\hline \multirow[t]{2}{*}{96} & $09-13-90$ & 11.2 & 620 & 7.3 & 16.0 & 186 & 366 & 90 & 13 & 20 \\
\hline & $02-21-91$ & 13.5 & 970 & 7.2 & 9.0 & -- & - & -- & -- & - \\
\hline \multirow[t]{2}{*}{97} & $09-14-90$ & 26.0 & 720 & 7.5 & 15.0 & -- & -- & -- & -- & -- \\
\hline & $02-22-91$ & 36.1 & 655 & 7.3 & 13.0 & - & -- & -- & -- & -- \\
\hline \multirow[t]{2}{*}{98} & $09-17-90$ & 32.0 & 833 & 7.3 & 14.0 & -- & -- & -- & -- & -- \\
\hline & $02-25-91$ & 51.4 & 1,160 & 6.9 & 13.5 & -- & - & -- & -- & -- \\
\hline \multirow[t]{2}{*}{99} & $09-18-90$ & 37.3 & 746 & 7.3 & 14.5 & -- & -- & -- & -- & -- \\
\hline & $02-20-91$ & 66.7 & 1,110 & 7.0 & 14.0 & -- & -- & - & -- & -- \\
\hline \multirow[t]{2}{*}{100} & $09-17-90$ & -- & 613 & 7.4 & 14.0 & -- & - & -- & -- & -- \\
\hline & $02-21-91$ & -- & 730 & 7.3 & 14.5 & - & -- & - & - & - \\
\hline 101 & $09-12-90$ & 20.7 & 675 & 7.3 & 15.5 & -- & - & -. & -- & -- \\
\hline 102 & $09-12-90$ & 7.5 & 640 & 7.3 & 14.5 & 197 & 377 & 82 & 16 & 28 \\
\hline \multirow[t]{2}{*}{103} & $09-20-90$ & 15.9 & 832 & 7.0 & 17.5 & 271 & 505 & 120 & 17 & 31 \\
\hline & $02-22-91$ & -- & 765 & 7.2 & 10.0 & -- & -- & -- & -- & - \\
\hline 104 & $09-14-90$ & 8.0 & 625 & 7.4 & 14.0 & 199 & 372 & 86 & 15 & 22 \\
\hline 105 & $09-18-90$ & 34.3 & 1,020 & 7.4 & 15.0 & 264 & 605 & 82 & 18 & 120 \\
\hline \multirow[t]{2}{*}{106} & $09-13-90$ & 17.5 & 840 & 7.0 & 14.5 & -- & -- & -- & -- & -- \\
\hline & $02-21-91$ & 29.5 & 700 & 6.9 & 13.5 & -- & -- & -- & - & -- \\
\hline 107 & $09-17-90$ & -- & 790 & 7.3 & 16.0 & 247 & 477 & 100 & 23 & 38 \\
\hline 108 & $09-12-90$ & -- & 615 & 7.3 & 17.5 & -- & - & -- & -- & -- \\
\hline \multirow[t]{2}{*}{109} & $09-13-90$ & 9.2 & 862 & 7.0 & 17.0 & -- & -- & -- & -- & -- \\
\hline & $02-22-91$ & 10.4 & 795 & 7.0 & 11.5 & -- & -- & - & -- & -- \\
\hline 110 & $09-11-90$ & -- & 775 & 7.1 & 15.0 & -- & -- & -. & - & - \\
\hline
\end{tabular}


Table 2. Water-level and water-quality measurements and nearby soil-gas-methane concentrations for selected water wells and springs--Continued

\begin{tabular}{|c|c|c|c|c|c|c|c|c|c|c|}
\hline $\begin{array}{l}\text { Num- } \\
\text { ber } \\
\text { on } \\
\text { plate } \\
1\end{array}$ & $\begin{array}{c}\text { Date } \\
\text { sampled }\end{array}$ & $\begin{array}{l}\text { Water } \\
\text { level }\end{array}$ & $\begin{array}{c}\text { Speclific } \\
\text { con- } \\
\text { ductance } \\
(\mu \mathrm{S} / \mathrm{cm})\end{array}$ & $\begin{array}{c}\mathrm{pH} \\
\text { (standard } \\
\text { unlts) }\end{array}$ & $\begin{array}{c}\text { Temper- } \\
\text { ature, } \\
\text { water } \\
\left({ }^{\circ} \mathrm{C}\right)\end{array}$ & $\begin{array}{l}\text { Alkalinity } \\
\text { dis- } \\
\text { solved, } \\
\text { onsite, } \\
\text { IT } \\
\left(\mathrm{mg}^{2} \mathrm{~L} \mathrm{as}\right. \\
\left.\mathrm{CaCO}_{3}\right)\end{array}$ & $\begin{array}{l}\text { Dis- } \\
\text { solved } \\
\text { solids, } \\
\text { sum of } \\
\text { constit- } \\
\text { uents } \\
\text { (mg/L) }\end{array}$ & $\begin{array}{c}\text { Caiclum, } \\
\text { dissolved } \\
\text { (mg/L as } \\
\text { Ca) }\end{array}$ & $\begin{array}{l}\text { Magne- } \\
\text { slum, } \\
\text { dissolved } \\
\text { (mg/L as } \\
\mathbf{M g})\end{array}$ & $\begin{array}{c}\text { Sodium, } \\
\text { dissolved } \\
\text { (mg/L as } \\
\mathrm{Na})\end{array}$ \\
\hline 111 & $09-19-90$ & 20.8 & 820 & 7.1 & 16.0 & -- & - & -- & - & -- \\
\hline \multirow[t]{2}{*}{112} & $09-17-90$ & -- & 748 & 7.1 & 15.0 & -- & -- & -- & -- & -- \\
\hline & $02-20-91$ & -. & 752 & 6.7 & 14.5 & -- & -- & - & - & -- \\
\hline \multirow[t]{2}{*}{113} & $09-12-90$ & 25.1 & 715 & 7.4 & 14.0 & - & -- & -- & -- & -- \\
\hline & $02-21-91$ & 41.6 & 894 & 7.2 & 14.0 & -- & -- & -- & -- & -- \\
\hline 114 & $09-11-90$ & 12.3 & 2,790 & 7.4 & 15.5 & 247 & 2,090 & 230 & 41 & 390 \\
\hline \multirow[t]{2}{*}{115} & $09-11-90$ & 3.2 & 578 & 7.4 & 17.5 & -- & -- & -- & -- & -- \\
\hline & $02-25-91$ & 6.4 & 675 & 7.0 & 12.0 & -- & -- & -- & -- & -- \\
\hline \multirow[t]{2}{*}{116} & $09-17-90$ & 22.7 & 631 & 7.3 & 16.5 & -- & -- & -- & -- & -- \\
\hline & $02-22-91$ & 32.8 & 730 & 7.1 & 15.0 & -- & -- & -- & -- & - \\
\hline 117 & $10-15-90$ & - & 623 & 7.4 & 17.0 & -- & -- & -- & -- & -- \\
\hline 118 & $09-10-90$ & 5.2 & 2,250 & 7.4 & 15.0 & -- & -- & -- & -- & -- \\
\hline \multirow[t]{2}{*}{119} & $09-20-90$ & 30.3 & 643 & 7.4 & 15.0 & -- & -- & -- & -- & -- \\
\hline & $02-20-91$ & 43.4 & 605 & 6.9 & 15.0 & -- & -- & -- & - & - \\
\hline 120 & $09-18-90$ & 17.5 & 1,950 & 7.4 & 15.5 & 247 & 805 & 57 & 7.8 & 230 \\
\hline \multirow[t]{2}{*}{121} & $09-14-90$ & 25.0 & 745 & 7.2 & 16.0 & 267 & 433 & 110 & 17 & 24 \\
\hline & $02-20-91$ & 32.2 & 765 & 6.9 & 15.0 & -- & -- & -- & -- & - \\
\hline 122 & $09-19-90$ & 26.7 & 684 & 7.3 & 16.0 & - & -- & -- & -- & -- \\
\hline 123 & $09-27-90$ & 4.8 & 1,660 & 7.1 & 18.0 & 289 & 1,000 & 160 & 40 & 120 \\
\hline 124 & $09-19-90$ & 6.7 & 690 & 7.5 & 14.5 & 223 & 426 & 98 & 15 & 26 \\
\hline \multirow[t]{2}{*}{125} & $09-17-90$ & 13.9 & 665 & 7.2 & 16.5 & -- & - & -. & -- & -- \\
\hline & $02-25-91$ & 23.8 & 770 & 6.7 & 14.5 & -- & -- & - & -- & -- \\
\hline 126 & $09-19-90$ & 8.4 & 688 & 7.3 & 16.0 & -- & - & -- & -- & - \\
\hline 127 & $09-20-90$ & -- & 10,000 & 8.2 & 13.0 & 129 & 6,020 & 120 & 4.7 & 2,000 \\
\hline 128 & $09-20-90$ & - & 730 & 7.4 & 13.0 & 243 & 428 & 100 & 15 & 28 \\
\hline 129 & $09-24-90$ & -- & 3,400 & 7.1 & 14.0 & -- & -- & -- & -- & -- \\
\hline 130 & $09-24-90$ & 17.6 & 1,110 & 7.1 & 16.0 & - & -- & -- & -- & -- \\
\hline 131 & $09-20-90$ & 6.0 & 985 & 7.2 & 14.5 & -- & -- & - & -- & -- \\
\hline 132 & $09-27-90$ & -- & 710 & 7.2 & 16.0 & - & -- & - & -- & -- \\
\hline 133 & $09-24-90$ & 21.1 & 870 & 7.2 & 14.0 & -- & -- & -- & -- & -- \\
\hline 134 & $09-27-90$ & -- & 850 & 6.9 & 14.5 & -- & -- & -- & -- & -- \\
\hline 135 & $09-27-90$ & -- & 665 & 7.2 & 16.0 & -- & -- & -- & -- & -- \\
\hline
\end{tabular}


Table 2. Water-level and water-quality measurements and nearby soil-gas-methane concentrations for selected water wells and springs--Continued

\begin{tabular}{|c|c|c|c|c|c|c|c|c|c|c|}
\hline $\begin{array}{l}\text { Num- } \\
\text { ber } \\
\text { on } \\
\text { plate } \\
1\end{array}$ & $\begin{array}{c}\text { Date } \\
\text { sampled }\end{array}$ & $\begin{array}{c}\text { Water } \\
\text { level }\end{array}$ & $\begin{array}{c}\text { Specific } \\
\text { con- } \\
\text { ductance } \\
(\mu \mathrm{S} / \mathrm{cm})\end{array}$ & $\begin{array}{c}\text { pH } \\
\text { (standard } \\
\text { units) }\end{array}$ & $\begin{array}{c}\text { Temper- } \\
\text { ature, } \\
\text { water } \\
\left({ }^{\circ} \mathrm{C}\right)\end{array}$ & $\begin{array}{l}\text { Alkailinity } \\
\text { dis- } \\
\text { solved, } \\
\text { onsite, } \\
\text { IT } \\
(\mathrm{mg} / \mathrm{L} \text { as } \\
\left.\mathrm{CaCO}_{3}\right)\end{array}$ & $\begin{array}{c}\text { Dis- } \\
\text { soived } \\
\text { solids, } \\
\text { sum of } \\
\text { constit- } \\
\text { uents } \\
\text { (mg/L) }\end{array}$ & $\begin{array}{l}\text { Caicium, } \\
\text { dissoived } \\
\text { (mg/L as } \\
\text { Ca) }\end{array}$ & $\begin{array}{l}\text { Magne- } \\
\text { sium, } \\
\text { dissolved } \\
\text { (mg/L as } \\
\text { Mg) }\end{array}$ & $\begin{array}{l}\text { Sodium, } \\
\text { dissoived } \\
\text { (mg/L as } \\
\mathrm{Na} \text { ) }\end{array}$ \\
\hline 136 & $09-27-90$ & 21.3 & 980 & 7.3 & 14.0 & 222 & 593 & 93 & 14 & 91 \\
\hline 137 & $09-27-90$ & 30.6 & 1,180 & 7.4 & 15.5 & - & - & - & -- & - \\
\hline 138 & $09-26-90$ & -- & 700 & 7.5 & 17.0 & 254 & 434 & 110 & 13 & 23 \\
\hline 139 & $10-01-90$ & 6.6 & 828 & 7.3 & 14.5 & - & - & - & - & -- \\
\hline 140 & $09-26-90$ & -- & 680 & 7.3 & 17.0 & -- & - & - & - & -- \\
\hline 141 & $09-24-90$ & - & 2,500 & 7.1 & 14.0 & - & -- & -- & -- & -- \\
\hline 142 & $10-01-90$ & 7.3 & 910 & 7.2 & 14.5 & - & -- & -- & -- & -- \\
\hline 143 & $09-24-90$ & 23.0 & 600 & 7.3 & 16.5 & - & - & -- & -- & -- \\
\hline 144 & $10-01-90$ & 20.8 & 1,320 & 7.3 & 15.0 & 236 & 964 & 170 & 27 & 98 \\
\hline 145 & $10-01-90$ & 13.4 & 1,650 & 7.2 & 15.0 & - & -- & -- & - & -- \\
\hline 146 & $09-24-90$ & 8.6 & 900 & 7.2 & 13.0 & 297 & 508 & 120 & 20 & 50 \\
\hline 147 & $10-01-90$ & 4.0 & 775 & 7.1 & 13.0 & - & - & - & - & -- \\
\hline 148 & $09-25-90$ & 16.7 & 828 & 7.1 & 14.5 & $\cdots$ & -- & -- & - & - \\
\hline 149 & $10-18-90$ & - & 795 & 7.1 & 15.0 & $\cdots$ & -- & -- & - & -- \\
\hline 150 & $10-02-90$ & 6.4 & 1,080 & 7.1 & 14.0 & 311 & - & - & - & -- \\
\hline 151 & $09-25-90$ & 17.0 & 1,220 & 7.0 & 15.5 & - & -- & -- & - & -- \\
\hline 152 & $09-25-90$ & -- & 870 & 7.2 & 15.5 & -- & -- & -- & - & -- \\
\hline 153 & $09-26-90$ & -- & 1,140 & 7.1 & 14.5 & - & -- & -- & - & -- \\
\hline 154 & $09-25-90$ & 7.0 & 1,290 & 7.1 & 15.0 & 297 & 847 & 140 & 34 & 94 \\
\hline 155 & $10-01-90$ & 9.7 & 1,600 & 7.1 & 14.0 & - & - & $\ldots$ & - & -- \\
\hline 156 & $10-04-90$ & 5.9 & 2,900 & 7.2 & 18.0 & 244 & 2,020 & 150 & 21 & 480 \\
\hline 157 & $09-26-90$ & 5.2 & 1,810 & 7.3 & 14.0 & 274 & 1,160 & 150 & 40 & 170 \\
\hline 158 & $10-02-90$ & 8.1 & 2,140 & 7.3 & 16.0 & 306 & 1,510 & 190 & 32 & 260 \\
\hline 159 & $10-02-90$ & 13.3 & 2,870 & 7.6 & 14.0 & - & -- & - & -- & -- \\
\hline 160 & $09-26-90$ & 21.1 & 873 & 7.2 & 16.0 & - & -- & -- & - & - \\
\hline 161 & $10-03-90$ & -- & 720 & 7.2 & 14.0 & - & -- & -- & -- & -- \\
\hline 162 & $10-02-90$ & 27.4 & 740 & 7.1 & 16.0 & $\cdots$ & -- & -- & - & -- \\
\hline 163 & $10-03-90$ & 6.9 & 800 & 7.1 & 16.0 & - & - & - & -- & -- \\
\hline 164 & $10-03-90$ & 46.4 & 420 & 7.3 & 14.0 & - & -- & - & - & - \\
\hline 165 & $10-02-90$ & 23.2 & 905 & 7.1 & 14.0 & -- & -- & - & -- & -- \\
\hline
\end{tabular}


Table 2. Water-level and water-quality measurements and nearby soil-gas-methane concentrations for selected water wells and springs--Continued

\begin{tabular}{|c|c|c|c|c|c|c|c|c|c|c|}
\hline $\begin{array}{l}\text { Num- } \\
\text { ber } \\
\text { on } \\
\text { plate } \\
1\end{array}$ & $\begin{array}{c}\text { Date } \\
\text { sampled }\end{array}$ & $\begin{array}{l}\text { Water } \\
\text { level }\end{array}$ & $\begin{array}{c}\text { Specific } \\
\text { con- } \\
\text { ductance } \\
(\mu \mathrm{S} / \mathrm{cm})\end{array}$ & $\begin{array}{c}\text { pH } \\
\text { (standard } \\
\text { units) }\end{array}$ & $\begin{array}{c}\text { Temper- } \\
\text { ature, } \\
\text { water } \\
\left({ }^{\circ} \mathrm{C}\right)\end{array}$ & $\begin{array}{l}\text { Alkallnity } \\
\text { dls- } \\
\text { solved, } \\
\text { onsite, } \\
\text { IT } \\
\text { (mg/l as } \\
\mathrm{CaCO}_{3} \text { ) }\end{array}$ & $\begin{array}{l}\text { Dis- } \\
\text { solved } \\
\text { sollds, } \\
\text { sum of } \\
\text { constlt- } \\
\text { uents } \\
\text { (mg/L) }\end{array}$ & $\begin{array}{c}\text { Caicium, } \\
\text { dissolved } \\
(\mathrm{mg} / \mathrm{L} \text { as } \\
\text { Ca) }\end{array}$ & $\begin{array}{l}\text { Magne- } \\
\text { slum, } \\
\text { dlssolved } \\
\text { (mg/L as } \\
\text { Mg) }\end{array}$ & $\begin{array}{c}\text { Sodium, } \\
\text { dissolved } \\
\text { (mg/L as } \\
\mathrm{Na})\end{array}$ \\
\hline 166 & $10-03-90$ & 11.2 & 690 & 7.3 & 14.5 & -- & -- & -- & -- & -- \\
\hline 167 & $10-04-90$ & -- & 915 & 7.1 & 13.0 & -- & - & -- & - & - \\
\hline 168 & $09-26-90$ & 26.0 & 973 & 7.1 & 14.5 & -- & - & - & -- & - \\
\hline 169 & $10-04-90$ & - & 710 & 7.3 & 15.5 & - & -- & - & -. & - \\
\hline 170 & $10-03-90$ & - & 1,020 & 7.3 & 14.0 & - & - & - & -- & -- \\
\hline 171 & $10-03-90$ & 12.9 & 785 & 7.2 & 14.5 & - & - & - & - & - \\
\hline 172 & $10-05-90$ & 38.8 & 775 & 7.2 & 13.5 & - & - & - & - & -- \\
\hline 173 & $10-04-90$ & 18.5 & 1,420 & 7.1 & 14.0 & - & -- & -- & - & -- \\
\hline 174 & $10-04-90$ & 38.2 & 1,020 & 7.2 & 14.0 & 301 & 607 & 110 & 20 & 89 \\
\hline 175 & $10-05-90$ & -- & 1,070 & 7.0 & 15.0 & -- & -- & -- & -- & -- \\
\hline 176 & $10-18-90$ & -- & 7,000 & 7.2 & 15.0 &.- & -- & -- & -- & -- \\
\hline 177 & $10-09-90$ & -- & 1,360 & 7.2 & 14.0 & 288 & 916 & 140 & 24 & 130 \\
\hline 178 & $10-05-90$ & 26.7 & 1,040 & 7.1 & 14.5 & -- & - & -- & -- & - \\
\hline 179 & $10-15-90$ & 38.3 & 1,200 & 7.2 & 14.0 & -- & - & -- & -- & -- \\
\hline 180 & $10-18-90$ & 10.3 & 923 & 7.2 & 15.0 & -- & - & - & -- & -- \\
\hline 181 & $10-18-90$ & 17.5 & 3,320 & 7.1 & 15.0 & - & - & - & -- & - \\
\hline 182 & $10-18-90$ & 32.6 & 2,280 & 7.2 & 14.0 & -- & - & - & -- & $\cdots$ \\
\hline 183 & $10-03-90$ & 3.3 & 1,100 & 7.2 & 12.5 & - & - & - & -- & -- \\
\hline 184 & $10-09-90$ & 8.8 & 2,740 & 7.1 & 15.0 & 395 & 2,030 & 220 & 60 & 320 \\
\hline 185 & $10-16-90$ & -- & 858 & 7.2 & 14.0 & -- & -- & -- & - & - \\
\hline 186 & $10-16-90$ & -- & 1,770 & 7.2 & 14.0 & - & - & - & -- & -- \\
\hline 187 & $10-05-90$ & -- & 1,010 & 7.1 & 14.5 & - & -- & -- & -- & -- \\
\hline 188 & $10-15-90$ & 5.9 & 2,170 & 7.1 & 15.0 & - & - & - & -- & - \\
\hline 189 & $10-16-90$ & 23.9 & 754 & 7.4 & 13.5 & -- & - & - & -- & -- \\
\hline 190 & $10-09-90$ & 3.9 & 1,870 & 7.2 & 14.0 & -- & - & - & - & - \\
\hline 191 & $10-09-90$ & 16.6 & 2,740 & 7.2 & 15.0 & -- & -- & - & -- & -- \\
\hline 192 & $10-05-90$ & 20.4 & 2,150 & 7.3 & 15.0 & 154 & 1,580 & 200 & 37 & 230 \\
\hline 193 & $10-15-90$ & -- & 750 & 7.2 & 15.0 & - & -- & -- & - & - \\
\hline 194 & $10-16-90$ & 19.7 & 1,160 & 7.2 & 14.0 & - & -- & -- & - & -- \\
\hline 195 & $10-15-90$ & -- & 925 & 7.2 & 15.0 & - & - & -- & -- & -- \\
\hline
\end{tabular}


Table 2. Water-level and water-quality measurements and nearby soil-gas-methane concentrations for selected water wells and springs--Continued

\begin{tabular}{|c|c|c|c|c|c|c|c|c|c|c|}
\hline $\begin{array}{l}\text { Num- } \\
\text { ber } \\
\text { on } \\
\text { plate } \\
1\end{array}$ & $\begin{array}{c}\text { Date } \\
\text { sampled }\end{array}$ & $\begin{array}{c}\text { Water } \\
\text { level }\end{array}$ & $\begin{array}{c}\text { Specific } \\
\text { con- } \\
\text { ductance } \\
(\mu \mathrm{S} / \mathrm{cm})\end{array}$ & $\begin{array}{c}\text { pH } \\
\text { (standard } \\
\text { units) }\end{array}$ & $\begin{array}{c}\text { Temper- } \\
\text { ature, } \\
\text { water } \\
\left({ }^{\circ} \mathrm{C}\right)\end{array}$ & $\begin{array}{l}\text { Alkallnity } \\
\text { dls- } \\
\text { soived, } \\
\text { onsite, } \\
\text { IT } \\
\left(\mathrm{mg}^{2} \mathrm{~L} \text { as }\right. \\
\left.\mathrm{CaCO}_{3}\right)\end{array}$ & $\begin{array}{c}\text { Dls- } \\
\text { solved } \\
\text { sollds, } \\
\text { sum of } \\
\text { constit- } \\
\text { uents } \\
\text { (mg/L) }\end{array}$ & $\begin{array}{l}\text { Calcium, } \\
\text { dissolved } \\
(\mathrm{mg} / \mathrm{L} \text { as } \\
\text { Ca) }\end{array}$ & $\begin{array}{l}\text { Magne- } \\
\text { slum, } \\
\text { dlssolved } \\
\text { (mg/L as } \\
\mathrm{Mg} \text { ) }\end{array}$ & $\begin{array}{c}\text { Sodium, } \\
\text { dissolved } \\
\text { (mg/L as } \\
\mathrm{Na})\end{array}$ \\
\hline 196 & $10-17-90$ & -- & 838 & 7.1 & 12.0 & -- & -- & -- & -- & $\overline{--}$ \\
\hline 197 & $10-16-90$ & 5.2 & 959 & 7.2 & 14.0 & -- & -- & -- & -- & -- \\
\hline 198 & $10-17-90$ & 4.3 & 959 & 7.1 & 13.5 & -- & - & -- & -- & - \\
\hline 199 & $10-17-90$ & 4.7 & 1,180 & 7.2 & 14.0 & -- & -- & -- & -- & - \\
\hline 200 & $10-18-90$ & 31.3 & 910 & 7.1 & 15.0 & -- & -- & -- & -- & -- \\
\hline 201 & $10-16-90$ & -- & 1,590 & 7.1 & 14.0 & -- & - & -- & -- & -- \\
\hline 202 & $10-17-90$ & 3.7 & 1,300 & 7.1 & 14.0 & -- & -- & -- & -- & -- \\
\hline 203 & $10-17-90$ & 2.4 & 1,390 & 7.1 & 16.5 & 354 & 997 & 210 & 22 & 78 \\
\hline 204 & $10-17-90$ & 4.8 & 725 & 7.2 & 15.0 & - & -- & -- & - & -- \\
\hline 205 & $10-16-90$ & 26.9 & 2,200 & 6.9 & 13.5 & -- & -- & -- & -- & -- \\
\hline
\end{tabular}

\begin{tabular}{|c|c|c|c|c|c|c|c|c|c|c|}
\hline $\begin{array}{c}\text { Num- } \\
\text { ber } \\
\text { on } \\
\text { piate } \\
1\end{array}$ & $\begin{array}{c}\text { Date } \\
\text { sampled }\end{array}$ & $\begin{array}{l}\text { Potas- } \\
\text { slum, } \\
\text { dis- } \\
\text { solved } \\
\text { (mg/L as } \\
\text { K) }\end{array}$ & $\begin{array}{c}\text { Sulfate, } \\
\text { dls- } \\
\text { solved } \\
\text { (mg/L as } \\
\left.\mathrm{SO}_{4}\right)\end{array}$ & $\begin{array}{c}\text { Chioride, } \\
\text { dis- } \\
\text { solved } \\
\text { (mg/L as } \\
\text { Ci) }\end{array}$ & $\begin{array}{c}\text { Bromide, } \\
\text { dis- } \\
\text { solved } \\
\text { (mg/L as } \\
\mathrm{Br} \text { ) }\end{array}$ & $\begin{array}{c}\text { Silica, } \\
\text { dis- } \\
\text { solved } \\
\text { (mg/L as } \\
\left.\text { SiO }_{2}\right)\end{array}$ & $\begin{array}{c}\text { Iron, } \\
\text { dis- } \\
\text { solved } \\
(\mu \mathrm{g} / \mathrm{L} \text { as } \\
\mathrm{Fe})\end{array}$ & $\begin{array}{c}\text { Manga- } \\
\text { nese, } \\
\text { dls- } \\
\text { solved } \\
(\mu g / L \text { as } \\
\text { Mn })\end{array}$ & $\begin{array}{l}\text { Methane, } \\
\text { dis- } \\
\text { solved } \\
\text { (mg/L) }\end{array}$ & $\begin{array}{c}\text { Methane, } \\
\text { in } \\
\text { soll gas } \\
\left(\mathrm{mg} / \mathrm{L}_{\mathrm{g}}\right)\end{array}$ \\
\hline 1 & $08-28-90$ & -- & - & - & -- & - & -- & - & $<.005$ & $<.005$ \\
\hline 2 & $08-28-90$ & .79 & 190 & 21 & .14 & 9.2 & 5 & 4 & $<.005$ & - \\
\hline 3 & $08-27-90$ & - & -- & -- & -- & - & -- & -- & .068 & $<.005$ \\
\hline 4 & $08-27-90$ & .25 & 44 & 14 & .12 & 12 & 4 & 12 & 11 & $<.005$ \\
\hline 5 & $10-19-90$ & -- & -- & -- & -- & -- & -- & -- & $<.005$ & $<.005$ \\
\hline 6 & $09-05-90$ & -- & -- & - & -. & -- & -- & -- & $<.005$ & $<.005$ \\
\hline 7 & $08-28-90$ & .29 & 69 & 46 & .37 & 12 & 17 & 3 & 2.8 & $<.005$ \\
\hline 8 & $08-27-90$ & -- & -- & -- & -- & -- & -- & -- & $<.005$ & $<.005$ \\
\hline 9 & $08-29-90$ & .29 & 80 & 26 & .09 & 11 & $<3$ & 4 & .010 & $<.005$ \\
\hline 10 & $08-27-90$ & .45 & 100 & 35 & .12 & 9.1 & 9 & 2 & $<.005$ & -- \\
\hline 11 & $08-29-90$ & -- & -- & -- & -- & - & -- & -- & $<.005$ & $<.005$ \\
\hline 12 & $08-24-90$ & -- & -- & - & - & -- & -- & -- & $<.005$ & $<.005$ \\
\hline 13 & $08-24-90$ & 2.7 & 55 & 23 & .19 & 16 & 18 & 3 & $<.005$ & $<.005$ \\
\hline 14 & $08-21-90$ & .34 & 42 & 88 & .14 & 10 & 19 & 7 & 16 & $<.005$ \\
\hline 15 & $11-19-90$ & -- & -. & -- & -- & -- & -- & -- & .040 & $<.005$ \\
\hline
\end{tabular}


Table 2. Water-level and water-quality measurements and nearby soil-gas-methane concentrations for selected water wells and springs--Continued

\begin{tabular}{|c|c|c|c|c|c|c|c|c|c|c|}
\hline $\begin{array}{c}\text { Num- } \\
\text { ber } \\
\text { on } \\
\text { plate } \\
1\end{array}$ & $\begin{array}{c}\text { Date } \\
\text { sampled }\end{array}$ & $\begin{array}{l}\text { Potas- } \\
\text { sium, } \\
\text { dis- } \\
\text { solved } \\
\text { (mg/L as } \\
\text { K) }\end{array}$ & $\begin{array}{l}\text { Sulfate, } \\
\text { dis- } \\
\text { solved } \\
\left(\mathrm{mg}^{\prime} \mathrm{L} \text { as }\right. \\
\left.\mathrm{SO}_{4}\right)\end{array}$ & $\begin{array}{l}\text { Chloride, } \\
\text { dis- } \\
\text { solved } \\
\text { (mg/L as } \\
\text { Cl) }\end{array}$ & $\begin{array}{c}\text { Bromide, } \\
\text { dis- } \\
\text { solved } \\
\text { (mg/Las } \\
\text { Br) }\end{array}$ & $\begin{array}{l}\text { Silica, } \\
\text { dis- } \\
\text { solved } \\
\left(\mathrm{mg}^{\prime} / \mathrm{Las}\right. \\
\left.\mathrm{SiO}_{2}\right)\end{array}$ & $\begin{array}{c}\text { Iron, } \\
\text { dis- } \\
\text { solved } \\
(\mu g / \mathrm{L} \text { as } \\
\text { Fe })\end{array}$ & $\begin{array}{l}\text { Manga- } \\
\text { nese, } \\
\text { dis- } \\
\text { solved } \\
(\mu g / L \text { as } \\
\text { Mn) }\end{array}$ & $\begin{array}{l}\text { Methane, } \\
\text { dis- } \\
\text { solved } \\
\text { (mg/L) }\end{array}$ & $\begin{array}{c}\text { Methane, } \\
\text { in } \\
\text { soil gas } \\
\left(\mathrm{mg} / \mathrm{L}_{\mathrm{g}}\right)\end{array}$ \\
\hline 16 & $08-24-90$ & 1.6 & 27 & 11 & 0.16 & 18 & $<3$ & $<1$ & $<0.005$ & -- \\
\hline 17 & $08-24-90$ & -- & -- & -- & -- & -- & -- & -- & $<.005$ & $<.005$ \\
\hline 18 & $08-24-90$ & -- & -- & -- & -- & -- & -- & -- & $<.005$ & $<.005$ \\
\hline 19 & $08-21-90$ & -- & -- & -- & -- & -- & -- & -- & $<.005$ & $<.005$ \\
\hline 20 & $08-23-90$ & 1.3 & 80 & 22 & .18 & 11 & 4 & 6 & .54 & $<.005$ \\
\hline 21 & $08-23-90$ & -- & -- & -- & -- & -- & -- & -- & $<.005$ & $<.005$ \\
\hline 22 & $08-23-90$ & -- & -- & -- & -- & -- & -- & -- & $<.005$ & $<.005$ \\
\hline 23 & $08-23-90$ & -- & -- & -- & -- & -- & -- & -- & $<.005$ & $<.005$ \\
\hline 24 & $08-22-90$ & -- & -- & -- & -- & -- & -- & -- & $<.005$ & $<.005$ \\
\hline 25 & $08-21-90$ & .46 & 140 & 29 & .05 & 8.6 & 22 & 5 & .49 & $<.005$ \\
\hline 26 & $08-23-90$ & 1.6 & 83 & 20 & .10 & 9.6 & 6 & $<1$ & $<.005$ & $<.005$ \\
\hline 27 & $08-22-90$ & -- & -- & -- & -- & -- & -- & -- & $<.005$ & $<.005$ \\
\hline 28 & $08-20-90$ & - & -- & -- & -- & -- & -- & -- & $<.005$ & $<.005$ \\
\hline 29 & $08-22-90$ & .76 & 120 & 31 & .23 & 9.2 & 8 & $<1$ & .72 & $<.005$ \\
\hline 30 & $08-22-90$ & -- & -- & -- & -- & -- & -- & -- & $<.005$ & $<.005$ \\
\hline 31 & $08-22-90$ & -- & -- & -- & -- &.- & -. & -- & $<.005$ & $<.005$ \\
\hline 32 & $08-21-90$ & -- & -- & -- & -- & -- & -. & -- & $<.005$ & $<.005$ \\
\hline 33 & $08-23-90$ & -- & -- & -- & -- & -- & -- & -- & $<.005$ & $<.005$ \\
\hline 34 & $08-23-90$ & -- & -- & -. & -- & -. & -. & -- & $<.005$ & -- \\
\hline 35 & $08-22-90$ & 1.7 & 97 & 27 & .21 & 13 & $<3$ & $<1$ & $<.005$ & $<.005$ \\
\hline \multirow[t]{3}{*}{36} & $08-20-90$ & 1.8 & 74 & 20 & .16 & 9.0 & $<3$ & 10 & 19 & $<.005$ \\
\hline & $11-15-90$ & -- & -- & -- & -- & -- & -- & -- & 9.0 & -- \\
\hline & $03-06-91$ & -- & -. & -- & -- & -- & -- & -- & 4.5 & -- \\
\hline 37 & $08-16-90$ & -- & -- & -- & -- & -- & -- & -- & .006 & $<.005$ \\
\hline 38 & $08-15-90$ & 1.3 & 86 & 14 & .02 & 8.4 & 5 & 3 & 1.6 & $<.005$ \\
\hline 39 & $08-15-90$ & .49 & 160 & 150 & .34 & 9.8 & 13 & 12 & 5.0 & $<.005$ \\
\hline 40 & $08-15-90$ & -- & -- & -- & -- & -- & -- & -- & 2.4 & $<.005$ \\
\hline 41 & $08-17-90$ & 2.6 & 110 & 19 & .05 & 11 & 230 & 32 & .80 & $<.005$ \\
\hline 42 & $08-17-90$ & .95 & 280 & 67 & .26 & 9.5 & 170 & 81 & 5.3 & $<.005$ \\
\hline 43 & $08-16-90$ & 1.9 & 150 & 40 & .13 & 10 & 9 & $<1$ & 27 & $<.005$ \\
\hline 44 & $08-14-90$ & -- & -- & -- & -- & -- & -- & -- & $<.005$ & $<.005$ \\
\hline 45 & $08-14-90$ & -- & -- & -- & -- & -- & -- & -- & $<.005$ & $<.005$ \\
\hline
\end{tabular}


Table 2. Water-level and water-quality measurements and nearby soil-gas-methane concentrations for selected water wells and springs--Continued

\begin{tabular}{|c|c|c|c|c|c|c|c|c|c|c|}
\hline $\begin{array}{l}\text { Num- } \\
\text { ber } \\
\text { on } \\
\text { plate } \\
1\end{array}$ & $\begin{array}{l}\text { Date } \\
\text { sampled }\end{array}$ & $\begin{array}{l}\text { Potas- } \\
\text { sium, } \\
\text { dls- } \\
\text { solved } \\
\text { (mg/L as } \\
\text { K) }\end{array}$ & $\begin{array}{l}\text { Sulfate, } \\
\text { dls- } \\
\text { solved } \\
(\mathrm{mg} / \mathrm{L} \mathrm{as} \\
\left.\mathrm{SO}_{4}\right)\end{array}$ & $\begin{array}{l}\text { Chloride, } \\
\text { dis- } \\
\text { solved } \\
\text { (mg/Las } \\
\text { Cl) }\end{array}$ & $\begin{array}{c}\text { Bromide, } \\
\text { dis- } \\
\text { solved } \\
\text { (mg/L as } \\
\text { Br) }\end{array}$ & $\begin{array}{l}\text { Silica, } \\
\text { dis- } \\
\text { solved } \\
\text { (mg/L as } \\
\left.\mathrm{SIO}_{2}\right)\end{array}$ & $\begin{array}{c}\text { Iron, } \\
\text { dis- } \\
\text { solved } \\
(\mu \mathrm{g} / \mathrm{L} \text { as } \\
\mathrm{Fe})\end{array}$ & $\begin{array}{l}\text { Manga- } \\
\text { nese, } \\
\text { dis- } \\
\text { solved } \\
(\mu \mathrm{g} / \mathrm{L} \text { as } \\
\mathrm{Mn})\end{array}$ & $\begin{array}{l}\text { Methane, } \\
\text { dls- } \\
\text { solved } \\
\text { (mg/L) }\end{array}$ & $\begin{array}{c}\text { Methane, } \\
\text { In } \\
\text { soll gas } \\
\left.\text { (mg/L }{ }^{\prime}\right)\end{array}$ \\
\hline 46 & $08-16-90$ & 0.62 & 120 & 36 & 0.18 & 9.2 & 13 & 7 & 0.025 & $<0.005$ \\
\hline 47 & $08-14-90$ & -- & -- & -- & -- & -- & -- & -- & $<.005$ & $<.005$ \\
\hline 48 & $08-13-90$ & -- & -- & -- & -. & -- & -. & -- & $<.005$ & -- \\
\hline 49 & $08-10-90$ & 2.0 & 76 & 13 & .03 & 9.2 & $<3$ & $<1$ & $<.005$ & $<.005$ \\
\hline 50 & $08-08-90$ & .69 & 110 & 71 & .27 & 8.9 & $<3$ & 7 & .46 & $<.005$ \\
\hline 51 & $08-17-90$ & -- & -- & -- & - & -- & -- & -- & .031 & $<.005$ \\
\hline 52 & $08-09-90$ & .60 & 110 & 48 & .14 & 8.2 & $<3$ & 25 & 2.3 & $<.005$ \\
\hline 53 & $08-07-90$ & .94 & 280 & 42 & .20 & 9.4 & 11 & 6 & $<.005$ & $<.005$ \\
\hline 54 & $10-29-90$ & 2.1 & 130 & 35 & .05 & 11 & 28 & 3 & 28 & $<.005$ \\
\hline 55 & $10-29-90$ & 3.5 & 130 & 26 & .05 & 11 & 93 & 8 & .26 & $<.005$ \\
\hline 56 & $08-09-90$ & -- & -- & -- & -- & -- & - & -- & .007 & $<.005$ \\
\hline 57 & $10-10-90$ & 1.6 & 100 & 51 & .09 & 9.2 & 8 & 29 & .96 & $<.005$ \\
\hline 58 & $08-06-90$ & 1.4 & 44 & 350 & .87 & 10 & 620 & 230 & 7.4 & $<.005$ \\
\hline 59 & $10-25-90$ & 2.1 & 140 & 34 & .13 & 9.1 & 17 & 100 & 2.6 & $<.005$ \\
\hline 60 & $08-20-90$ & -- & -- & -- & -- & -- & -- & -- & .059 & $<.005$ \\
\hline 61 & $08-14-90$ & 2.7 & 120 & 17 & .05 & 11 & 910 & 380 & .26 & $<.005$ \\
\hline 62 & $08-29-90$ & -. & -- & -- & -- & -- & -. & -- & $<.005$ & $<.005$ \\
\hline 63 & $08-13-90$ & 1.4 & 320 & 41 & .10 & 12 & 19 & $<1$ & $<.005$ & $<.005$ \\
\hline 64 & $08-29-90$ & 2.8 & 910 & 300 & .73 & 11 & 120 & 88 & .10 & $<.005$ \\
\hline 65 & $08-30-90$ & 3.0 & 230 & 1,000 & .98 & 8.9 & 64 & 140 & .48 & $<.005$ \\
\hline 66 & $09-04-90$ & -- & -- & -- & -- & -- & -- & -- & .56 & $<.005$ \\
\hline 67 & $08-30-90$ & -- & -- & -- & -- & -- & -- &.- & $<.005$ & $<.005$ \\
\hline 68 & $08-30-90$ & -- & -- & -- & -- & -- & -- & -- & $<.005$ & $<.005$ \\
\hline 69 & $08-30-90$ & -- & -- & -. & -- & -. & -- & -- & $<.005$ & $<.005$ \\
\hline 70 & $08-30-90$ & .65 & 80 & 210 & .55 & 8.4 & 39 & 13 & 17 & $<.005$ \\
\hline 71 & $08-29-90$ & - & -- & - & -- & -- & -- & -- & $<.005$ & $<.005$ \\
\hline 72 & $09-10-90$ & -- & - & -- & -- & -- & -- & -- & $<.005$ & $<.005$ \\
\hline 73 & 09-04-90 & 2.3 & 12 & 1,200 & .89 & 7.5 & 250 & 74 & 39 & $<.005$ \\
\hline 74 & $09-05-90$ & -- & -- & -- & -- & -- & -- & -- & $<.005$ & $<.005$ \\
\hline 75 & $09-05-90$ & -- & - & -- & -- & -- & -- & -- & $<.005$ & $<.005$ \\
\hline 76 & $09-10-90$ & 2.6 & 490 & 260 & .45 & 11 & 55 & 52 & .29 & $<.005$ \\
\hline 77 & $09-05-90$ & 2.5 & 99 & 21 & .05 & 11 & 9 & 3 & $<.005$ & $<.005$ \\
\hline 78 & $09-11-90$ & -- & -- & -- & -- & -- & $-\cdots$ & -- & $<.005$ & $<.005$ \\
\hline 79 & $09-10-90$ & - & -- & -- & -- & -- & -- & -- & $<.005$ & $<.005$ \\
\hline 80 & 09-06-90 & -- & -- & -- & -- & -- & -- & -- & .009 & $<.005$ \\
\hline
\end{tabular}


Tabie 2. Water-level and water-quality measurements and nearby soil-gas-methane concentrations for selected water wells and springs--Continued

\begin{tabular}{|c|c|c|c|c|c|c|c|c|c|c|}
\hline $\begin{array}{l}\text { Num- } \\
\text { ber } \\
\text { on } \\
\text { plate } \\
1\end{array}$ & $\begin{array}{c}\text { Date } \\
\text { sampled }\end{array}$ & $\begin{array}{l}\text { Potas- } \\
\text { slum, } \\
\text { dls- } \\
\text { solved } \\
\text { (mg/L as } \\
\mathrm{K} \text { ) }\end{array}$ & $\begin{array}{l}\text { Sulfate, } \\
\text { dls- } \\
\text { solved } \\
(\mathrm{mg} / \mathrm{L} \text { as } \\
\left.\mathrm{SO}_{4}\right)\end{array}$ & $\begin{array}{l}\text { Chlorlde, } \\
\text { dis- } \\
\text { solved } \\
\text { (mg/L as } \\
\text { Cl) }\end{array}$ & $\begin{array}{l}\text { Bromlde, } \\
\text { dls- } \\
\text { solved } \\
\text { (mg/L as } \\
\text { Br) }\end{array}$ & $\begin{array}{l}\text { Sillca, } \\
\text { dls- } \\
\text { solved } \\
(\mathbf{m g} / \mathrm{L} \text { as } \\
\left.\mathrm{SiO}_{2}\right)\end{array}$ & $\begin{array}{c}\text { Iron, } \\
\text { dls- } \\
\text { solved } \\
\text { ( } \mu \mathrm{g} / \mathrm{L} \text { as } \\
\mathrm{Fe} \text { ) }\end{array}$ & $\begin{array}{l}\text { Manga- } \\
\text { nese, } \\
\text { dis- } \\
\text { solved } \\
\text { ( } \mu \mathrm{g} / \mathrm{L} \text { as } \\
\text { Mn) }\end{array}$ & $\begin{array}{c}\text { Methane, } \\
\text { dis- } \\
\text { solved } \\
\text { (mg/L) }\end{array}$ & $\begin{array}{c}\text { Methane, } \\
\text { In } \\
\text { soil gas } \\
\left.\text { (mg/L } L_{\text {g }}\right)\end{array}$ \\
\hline 81 & $09-07-90$ & - & -- & -- & -- & -- & -- & -- & $<0.005$ & $<0.005$ \\
\hline 82 & $09-06-90$ & 1.1 & 160 & 150 & .16 & 10 & 18 & 12 & 1.4 & $<.005$ \\
\hline 83 & $09-19-90$ & -- & -- & $\ldots$ & -- & -- & -. & -- & .90 & $<.005$ \\
\hline 84 & $09-11-90$ & 3.7 & 820 & 880 & 1.0 & 8.9 & 190 & 470 & 3.6 & .065 \\
\hline 85 & $09-11-90$ & -- & -- & -- & -- & -- & -- & -- & .98 & -- \\
\hline 86 & $09-06-90$ & -- & -- & -- & -- & -- & -- & -- & $<.005$ & $<.005$ \\
\hline 87 & $09-07-90$ & 2.4 & 110 & 19 & .05 & 13 & 3 & 1 & 1.2 & $<.005$ \\
\hline 88 & $09-07-90$ & -- & -. & -- & -- & -- & -- & -- & $<.005$ & $<.005$ \\
\hline \multirow[t]{2}{*}{89} & $09-12-90$ & -- & -- & $\cdots$ & -- & -. & -- & - & $<.005$ & $<.005$ \\
\hline & $02-20-91$ & - & -- & -- & -- & -- & -- & -- & $<.005$ & -- \\
\hline \multirow[t]{2}{*}{90} & $09-14-90$ & 2.4 & 110 & 23 & .06 & 10 & 2,000 & 620 & .009 & .005 \\
\hline & $02-22-91$ & - & -- & -- & -- & -- & -- & -- & $<.005$ & -- \\
\hline 91 & $09-13-90$ & - & -- & -- & -- & -- & -. & -- & $<.005$ & -- \\
\hline \multirow[t]{2}{*}{92} & $09-13-90$ & 5.9 & 120 & 100 & .13 & 13 & 49 & 1,400 & .71 & $<.005$ \\
\hline & $02-21-91$ & -- & -- & -- & -- & -- & -- & -- & $<.005$ & -- \\
\hline \multirow[t]{2}{*}{93} & $09-13-90$ & -- & -- & -- & -- & -- & -- & -- & $<.005$ & -- \\
\hline & $02-19-91$ & -- & -- & -- & -- & -- & -- & -- & $<.005$ & -- \\
\hline \multirow[t]{2}{*}{94} & $09-12-90$ & -- & -- & -- & -- & -- & -- & -- & $<.005$ & $<.005$ \\
\hline & $02-25-91$ & -- & -- & -- & -- & -- & -- & - & $<.005$ & -- \\
\hline 95 & $09-19-90$ & -. & -- & -- & -- & - & -- & -- & $<.005$ & $<.005$ \\
\hline \multirow[t]{2}{*}{96} & $09-13-90$ & 2.8 & 97 & 23 & .05 & 9.0 & 66 & 2 & $<.005$ & .5 \\
\hline & $02-21-91$ & -- & -- & -- & -- & -- & - & -- & .87 & - \\
\hline \multirow[t]{2}{*}{97} & $09-14-90$ & -- & -- & -- & .. & -- & -- & -- & $<.005$ & $<.005$ \\
\hline & $02-22-91$ & -- & -- & -- & -- & -- & -- & -- & $<.005$ & $-\cdot$ \\
\hline \multirow[t]{2}{*}{98} & $09-17-90$ & - & -- & $\cdots$ & -- & -. & -- & -- & $<.005$ & $<.005$ \\
\hline & $02-25-91$ & - & -- & -- & -- & -- & -- & -- & $<.005$ & -- \\
\hline \multirow[t]{2}{*}{99} & $09-18-90$ & -- & -- & -- & -- & -- & -- & -- & .007 & $<.005$ \\
\hline & $02-20-91$ & -- & -- & -- & -- & -- & -- & -- & .011 & -- \\
\hline \multirow[t]{2}{*}{100} & $09-17-90$ & -- & -- & -- & -. & -- & -. & -- & $<.005$ & $<.005$ \\
\hline & $02-21-91$ & -- & -- & -- & -- & -- & -- & -- & $<.005$ & - \\
\hline 101 & $09-12-90$ & -- & - & -- & -. & -- & -. & -- & $<.005$ & - \\
\hline 102 & $09-12-90$ & 3.1 & 98 & 22 & .05 & 10 & 170 & 3 & $<.005$ & $<.005$ \\
\hline \multirow[t]{2}{*}{103} & $09-20-90$ & 3.7 & 130 & 29 & .05 & 11 & 800 & 140 & .005 & $<.005$ \\
\hline & $02-22-91$ & -- & -- & -- & -- & -- & -- & -- & .041 & -- \\
\hline 104 & $09-14-90$ & 4.2 & 95 & 21 & .04 & 9.5 & 410 & 20 & .050 & $<.005$ \\
\hline 105 & $09-18-90$ & 2.2 & 130 & 82 & .11 & 11 & 560 & 640 & .080 & $<.005$ \\
\hline
\end{tabular}


Table 2. Water-level and water-quality measurements and nearby soil-gas-methane concentrations for selected water wells and springs--Continued

\begin{tabular}{|c|c|c|c|c|c|c|c|c|c|c|}
\hline $\begin{array}{c}\text { Num- } \\
\text { ber } \\
\text { on } \\
\text { plate } \\
1\end{array}$ & $\begin{array}{c}\text { Date } \\
\text { sampled }\end{array}$ & $\begin{array}{l}\text { Potas- } \\
\text { slum, } \\
\text { dis- } \\
\text { soived } \\
\text { (mg/L as } \\
\mathrm{K} \text { ) }\end{array}$ & $\begin{array}{l}\text { Sulfate, } \\
\text { dis- } \\
\text { solved } \\
\text { (mg/L as } \\
\left.\mathrm{sO}_{4}\right)\end{array}$ & $\begin{array}{c}\text { Chloride, } \\
\text { dis- } \\
\text { solved } \\
\text { ( } \mathrm{mg} / \mathrm{L} \text { as } \\
\text { Cl) }\end{array}$ & $\begin{array}{c}\text { Bromide, } \\
\text { dis- } \\
\text { solved } \\
\text { (mg/L as } \\
\mathrm{Br} \text { ) }\end{array}$ & $\begin{array}{l}\text { Siilca, } \\
\text { dis- } \\
\text { solved } \\
\left(\mathrm{mg}^{2} \mathrm{~L} \text { as }\right. \\
\left.\mathrm{SiO}_{2}\right)\end{array}$ & $\begin{array}{c}\text { Iron, } \\
\text { dis- } \\
\text { solved } \\
(\mu \mathrm{g} / \mathrm{L} \text { as } \\
\mathrm{Fe})\end{array}$ & $\begin{array}{l}\text { Manga- } \\
\text { nese, } \\
\text { dis- } \\
\text { soived } \\
(\mu \mathrm{g} / \mathrm{L} \text { as } \\
\mathrm{Mn})\end{array}$ & $\begin{array}{l}\text { Methane, } \\
\text { dis- } \\
\text { solved } \\
\text { (mg/L) }\end{array}$ & $\begin{array}{c}\text { Methane, } \\
\text { in } \\
\text { soil gas } \\
\left(\mathrm{mg} / \mathrm{L}_{\mathrm{g}}\right)\end{array}$ \\
\hline \multirow[t]{2}{*}{106} & $09-13-90$ & $\overline{--}$ & -- & -- & - & -- & -- & -- & $<0.005$ & -- \\
\hline & $02-21-91$ & -- & -- & -- & -- & -- & -- & -- & $<.005$ & -- \\
\hline 107 & $09-17-90$ & 2.2 & 130 & 25 & .07 & 11 & 8 & $<1$ & $<.005$ & $<.005$ \\
\hline 108 & $09-12-90$ & -- & -- & -- & -- & -- & -- & -- & $<.005$ & -- \\
\hline \multirow[t]{2}{*}{109} & $09-13-90$ & -- & -- & -- & -- & -- & -- & -- & $<.005$ & $<.005$ \\
\hline & $02-22-91$ & -- & -- & -. & -- & -- & -- & -- & $<.005$ & -- \\
\hline 110 & $09-11-90$ & -- & -- & -- & -- & -- & -- & -- & $<.005$ & $<.005$ \\
\hline 111 & $09-19-90$ & -. & -- & -- & -- & -- & -- & -- & $<.005$ & $<.005$ \\
\hline \multirow[t]{2}{*}{112} & $09-17-90$ & -- & -- & -- & -- & -- & -- & -- & $<.005$ & -- \\
\hline & $02-20-91$ & -- & -- & -- & -- & -- & -- & - & $<.005$ & -- \\
\hline \multirow[t]{2}{*}{113} & $09-12-90$ & -- & -- & -- & -- & -- & -- & -- & $<.005$ & -- \\
\hline & $02-21-91$ & -- & -- & -- & -- & -- & -- & -- & $<.005$ & -- \\
\hline 114 & $09-11-90$ & 3.5 & 1,200 & 66 & .25 & 8.6 & 370 & 3,600 & .20 & $<.005$ \\
\hline \multirow[t]{2}{*}{115} & $09-11-90$ & -- & -- & -- & -- & -. & -- & -- & $<.005$ & $<.005$ \\
\hline & $02-25-91$ & -- & -- & -- & -- & -- & -- & -. & $<.005$ & -- \\
\hline \multirow[t]{2}{*}{116} & $09-17-90$ & -- & -- & -- & -- & -- & -- & -- & $<.005$ & $<.005$ \\
\hline & $02-22-91$ & -. & -- & -- & -- & -- & -- & -- & .075 & -- \\
\hline 117 & $10-15-90$ & -- & -- & -- & -- & -- & -- & -- & $<.005$ & $<.005$ \\
\hline 118 & $09-10-90$ & -- & -- & -. & -- & -- & -- & -- & .13 & $<.005$ \\
\hline \multirow[t]{2}{*}{119} & $09-20-90$ & -- & -- & -- & -- & -- & -- & -- & $<.005$ & $<.005$ \\
\hline & $02-20-91$ & -- & -- & -- & -- & -- & -- & -- & $<.005$ & - \\
\hline 120 & $09-18-90$ & 2.2 & 160 & 190 & .19 & 8.9 & 690 & 110 & 15 & $<.005$ \\
\hline \multirow[t]{2}{*}{121} & $09-14-90$ & 2.1 & 86 & 21 & .04 & 11 & 6 & 1,500 & .63 & $<.005$ \\
\hline & $02-20-91$ & -- & -- & -. & -- & -- & -- & -- & $<.005$ & -- \\
\hline 122 & $09-19-90$ & -- & -- & -- & -- & -- & -- & -- & $<.005$ & $<.005$ \\
\hline 123 & $09-27-90$ & 5.8 & 250 & 240 & .26 & 10 & 28 & 530 & .45 & $<.005$ \\
\hline 124 & $09-19-90$ & 2.9 & 110 & 26 & .06 & 12 & 1,600 & 1,200 & 2.7 & $<.005$ \\
\hline \multirow[t]{2}{*}{125} & $09-17-90$ & -- & -- & -- & -- & -- & -- & -- & $<.005$ & $<.005$ \\
\hline & $02-25-91$ & -- & -- & -- & -- & -- & -- & -- & .54 & -- \\
\hline 126 & $09-19-90$ & -- & -- & -- & -- & -- & -- & -- & $<.005$ & $<.005$ \\
\hline 127 & $09-20-90$ & 4.9 & 2,100 & 1,700 & 1.6 & 9.0 & 140 & 490 & 33 & $<.005$ \\
\hline 128 & $09-20-90$ & 2.6 & 100 & 26 & .05 & 9.3 & 170 & 790 & 1.7 & $<.005$ \\
\hline 129 & $09-24-90$ & -- & - & -- & -- & -- & -. & -- & $<.005$ & $<.005$ \\
\hline 130 & $09-24-90$ & -. & -- & -- & -- & -- & -- & -- & $<.005$ & $<.005$ \\
\hline
\end{tabular}


Table 2. Water-level and water-quality measurements and nearby soil-gas-methane concentrations for selected water wells and springs--Continued

\begin{tabular}{|c|c|c|c|c|c|c|c|c|c|c|}
\hline $\begin{array}{l}\text { Num- } \\
\text { ber } \\
\text { on } \\
\text { plate } \\
1\end{array}$ & $\begin{array}{c}\text { Date } \\
\text { sampled }\end{array}$ & $\begin{array}{l}\text { Potas- } \\
\text { sium, } \\
\text { dis- } \\
\text { solved } \\
\text { (mg/L as } \\
\text { K) }\end{array}$ & $\begin{array}{l}\text { Sulfate, } \\
\text { dis- } \\
\text { solved } \\
(\mathrm{mg} / \mathrm{L} \text { as } \\
\left.\mathrm{SO}_{4}\right)\end{array}$ & $\begin{array}{l}\text { Chloride, } \\
\text { dis- } \\
\text { solved } \\
\text { (mg/L as } \\
\text { Cl) }\end{array}$ & $\begin{array}{l}\text { Bromide, } \\
\text { dis- } \\
\text { solved } \\
\text { (mg/L as } \\
\text { Br) }\end{array}$ & $\begin{array}{l}\text { Silica, } \\
\text { dis- } \\
\text { solved } \\
(\mathrm{mg} / \mathrm{L} \mathrm{as} \\
\left.\mathrm{SiO}_{2}\right)\end{array}$ & $\begin{array}{l}\text { Iron, } \\
\text { dis- } \\
\text { solved } \\
(\mu \mathrm{g} / \mathrm{L} \text { as } \\
\text { Fe) }\end{array}$ & $\begin{array}{l}\text { Manga- } \\
\text { nese, } \\
\text { dis- } \\
\text { solved } \\
\text { ( } \mu \text { g/L as } \\
\text { Mn) }\end{array}$ & $\begin{array}{l}\text { Methane, } \\
\text { dis- } \\
\text { solved } \\
\text { (mg/L) }\end{array}$ & $\begin{array}{l}\text { Methane, } \\
\text { in } \\
\text { soil gas } \\
\text { (mg/L })\end{array}$ \\
\hline 131 & $09-20-90$ & - & $-\cdot$ & $-\cdot$ & $-\cdot$ & -- & -- & -- & $<0.005$ & $<0.005$ \\
\hline 132 & $09-27-90$ & -- & -- & -- & -- & -- & -- & -- & $<.005$ & $<.005$ \\
\hline 133 & $09-24-90$ & - & -- & -- & -- & -- & -- & -- & $<.005$ & $<.005$ \\
\hline 134 & $09-27-90$ & - & -- & -- & -- & -- & -- & -- & $<.005$ & $<.005$ \\
\hline 135 & $09-27-90$ & -- & -- & -- & -- & - & -- & -- & $<.005$ & $<.005$ \\
\hline 136 & $09-27-90$ & 1.3 & 210 & 38 & .08 & 12 & 44 & 4 & 1.1 & $<.005$ \\
\hline 137 & $09-27-90$ & -- & -- & -- & -- & -- & -- & -- & .21 & $<.005$ \\
\hline 138 & $09-26-90$ & 1.8 & 100 & 22 & .05 & 10 & 910 & 260 & .38 & .032 \\
\hline 139 & $10-01-90$ & -- & -- & -- & -- & - & -- & -- & $<.005$ & $<.005$ \\
\hline 140 & $09-26-90$ & -- & -- & -- & -- & -- & -- & -- & $<.005$ & $<.005$ \\
\hline 141 & $09-24-90$ & - & -- & -- & -- & -- & -- & -- & $<.005$ & $<.005$ \\
\hline 142 & $10-01-90$ & - & -- & -- & -- & -- & -- & -- & $<.005$ & $<.005$ \\
\hline 143 & $09-24-90$ & -- & -- & -- & -- & -- & -- & -- & $<.005$ & $<.005$ \\
\hline 144 & $10-01-90$ & 1.1 & 470 & 41 & .12 & 12 & 1,000 & 2,200 & .014 & .005 \\
\hline 145 & $10-01-90$ & - & -- & $-\cdot$ & -- & -- & -- & -- & $<.005$ & $<.005$ \\
\hline 146 & $09-24-90$ & 2.6 & 110 & 17 & .06 & 9.9 & $<3$ & $<1$ & $<.005$ & $<.005$ \\
\hline 147 & $10-01-90$ & - & -- & -- & -- & -- & -- & -- & .005 & $<.005$ \\
\hline 148 & $09-25-90$ & - & -- & -- & -- & -- & -- & -- & $<.005$ & $<.005$ \\
\hline 149 & $10-18-90$ & -- & -- & - & -- & -- & -- & -- & .008 & $<.005$ \\
\hline 150 & $10-02-90$ & -- & $\cdots$ & -- & -- & -- & -- & -- & $<.005$ & $<.005$ \\
\hline 151 & $09-25-90$ & -- & -- & -- & -- & -- & -- & -- & $<.005$ & $<.005$ \\
\hline 152 & $09-25-90$ & -- & -- & -- & -- & -- & -- & -- & $<.005$ & $<.005$ \\
\hline 153 & $09-26-90$ & -- & -- & -- & -- & -- & -- & -- & $<.005$ & $<.005$ \\
\hline 154 & $09-25-90$ & 2.8 & 340 & 45 & .13 & 13 & 160 & 8 & $<.005$ & $<.005$ \\
\hline 155 & $10-01-90$ & -- & $-\cdot$ & -- & -- & -- & -- & -- & $<.005$ & $<.005$ \\
\hline 156 & $10-04-90$ & 4.0 & 1,100 & 110 & .16 & 12 & 73 & 840 & .11 & $<.005$ \\
\hline 157 & $09-26-90$ & 3.2 & 460 & 150 & .13 & 14 & 960 & 2,600 & .080 & $<.005$ \\
\hline 158 & $10-02-90$ & 4.9 & 730 & 100 & .12 & 12 & 1,100 & 750 & .024 & $<.005$ \\
\hline 159 & $10-02-90$ & - & -- & -- & -- & -- & -- & -- & $<.005$ & $<.005$ \\
\hline 160 & $09-26-90$ & -- & -- & -. & -- & -- & -- & -- & .018 & $<.005$ \\
\hline 161 & $10-03-90$ & -- & -- & -- & -- & - & - & -- & $<.005$ & $<.005$ \\
\hline 162 & $10-02-90$ & -- & -- & -- & $\cdots$ & -- & -- & -- & $<.005$ & $<.005$ \\
\hline 163 & $10-03-90$ & -- & -- & $-\cdot$ & -- & -- & -- & -- & $<.005$ & $<.005$ \\
\hline 164 & $10-03-90$ & -- & $\cdots$ & -- & $\cdots$ & -- & -- & -- & $<.005$ & $<.005$ \\
\hline 165 & $10-02-90$ & -- & -- & -- & -- & -- & -- & -- & $<.005$ & $<.005$ \\
\hline
\end{tabular}


Table 2. Water-level and water-quality measurements and nearby soil-gas-methane concentrations for selected water wells and springs--Continued

\begin{tabular}{|c|c|c|c|c|c|c|c|c|c|c|}
\hline $\begin{array}{l}\text { Num- } \\
\text { ber } \\
\text { on } \\
\text { plate } \\
1\end{array}$ & $\begin{array}{c}\text { Date } \\
\text { sampled }\end{array}$ & $\begin{array}{l}\text { Potas- } \\
\text { sium, } \\
\text { dis- } \\
\text { solved } \\
\text { (mg/L as } \\
\text { K) }\end{array}$ & $\begin{array}{c}\text { Sulfate, } \\
\text { dis- } \\
\text { solved } \\
\left(\mathrm{mg}^{2} / \mathrm{Las}\right. \\
\left.\mathrm{SO}_{4}\right)\end{array}$ & $\begin{array}{c}\text { Chloride, } \\
\text { dis- } \\
\text { solved } \\
\text { (mg/L as } \\
\text { Cl) }\end{array}$ & $\begin{array}{c}\text { Bromide, } \\
\text { dis- } \\
\text { solved } \\
\text { (mg/Las } \\
\mathrm{Br})\end{array}$ & $\begin{array}{c}\text { Silica, } \\
\text { dis- } \\
\text { solved } \\
(\mathbf{m g} / \mathrm{L} \text { as } \\
\left.\mathrm{SiO}_{2}\right)\end{array}$ & $\begin{array}{c}\text { Iron, } \\
\text { dis- } \\
\text { solved } \\
(\mu \mathrm{g} / \mathrm{L} \text { as } \\
\mathrm{Fe})\end{array}$ & $\begin{array}{c}\text { Manga- } \\
\text { nese, } \\
\text { dis- } \\
\text { solved } \\
(\mu \mathrm{g} / \mathrm{L} \text { as } \\
\text { Mn) }\end{array}$ & $\begin{array}{l}\text { Methane, } \\
\text { dis- } \\
\text { solved } \\
\text { (mg/L) }\end{array}$ & $\begin{array}{c}\text { Methane, } \\
\text { in } \\
\text { soll gas } \\
\left.\text { (mg/ } \mathrm{L}_{\mathrm{g}}\right)\end{array}$ \\
\hline 166 & $10-03-90$ & -- & -- & $\cdots$ & -- & -- & -- & - & $<0.005$ & $<0.005$ \\
\hline 167 & $10-04-90$ & -- & -- & -- & -- & -- & -- & -- & $<.005$ & $<.005$ \\
\hline 168 & $09-26-90$ & -- & -- & -- & -- & -- & -- & -- & $<.005$ & $<.005$ \\
\hline 169 & $10-04-90$ & -- & -- & -- & -- & -- & .- & -- & $<.005$ & $<.005$ \\
\hline 170 & $10-03-90$ & -. & -. & -- & -- & -- & -. & -- & $<.005$ & $<.005$ \\
\hline 171 & $10-03-90$ & -- & -- & -- & -- & -- & -- & -- & $<.005$ & $<.005$ \\
\hline 172 & $10-05-90$ & -- & -- & -- & .- & -- & -- & -- & .13 & $<.005$ \\
\hline 173 & $10-04-90$ & -- & -- & -- &.- & -- & -- & -- & $<.005$ & $<.005$ \\
\hline 174 & $10-04-90$ & 2.0 & 170 & 22 & .10 & 13 & 130 & 45 & 4.9 & $<.005$ \\
\hline 175 & $10-05-90$ & -- & -- & -- &.- & -- & -- & -- & $<.005$ & $<.005$ \\
\hline 176 & $10-18-90$ & -- & -- & -- & -- & -. & -- & -- & $<.005$ & $<.005$ \\
\hline 177 & $10-09-90$ & 1.9 & 400 & 35 & .13 & 12 & 190 & 24 & 1.5 & $<.005$ \\
\hline 178 & $10-05-90$ & -- & -- & -. & -. & -- & -- & -- & $<.005$ & $<.005$ \\
\hline 179 & $10-15-90$ & -- & -- & -- & -- &.- & -- & -- & $<.005$ & $<.005$ \\
\hline 180 & $10-18-90$ & -. & -- & -- & -- & .. & -- & -- & $<.005$ & $<.005$ \\
\hline 181 & $10-18-90$ & -- & -- & -- & -- & -- & -- & -- & $<.005$ & $<.005$ \\
\hline 182 & $10-18-90$ & -- &.- & -- & -- & -. & -- & -- & $<.005$ & $<.005$ \\
\hline 183 & $10-03-90$ & -- & - & -- & -- & -- & -- & -- & $<.005$ & $<.005$ \\
\hline 184 & $10-09-90$ & 3.3 & 1,100 & 88 & .14 & 4.6 & 230 & 1,300 & $<.005$ & $<.005$ \\
\hline 185 & $10-16-90$ & .. & -- & -- & -- & -- & -- & -- & $<.005$ & $<.005$ \\
\hline 186 & $10-16-90$ & .. & .- & .. & -- & .. & .. & -- & $<.005$ & $<.005$ \\
\hline 187 & $10-05-90$ &.- & -- & -. & .. & -. & -- & -- & $<.005$ & $<.005$ \\
\hline 188 & $10-15-90$ & -- & -- & -- & -- & -- & -- & -- & $<.005$ & $<.005$ \\
\hline 189 & $10-16-90$ & -. & - & -- & -. & - & -. & -. & $<.005$ & $<.005$ \\
\hline 190 & $10-09-90$ & -- & -- & -- & -- & -- & -- &.- & $<.005$ & $<.005$ \\
\hline 191 & $10-09-90$ & -- & -- & -- & -. & -- & -- & -. & $<.005$ & $<.005$ \\
\hline 192 & $10-05-90$ & 1.1 & 1,000 & 11 & .05 & 4.1 & 980 & 38 & .32 & $<.005$ \\
\hline 193 & $10-15-90$ & -- & -- & -- & -- & -- & -- & -. & $<.005$ & $<.005$ \\
\hline 194 & $10-16-90$ & -- & -- & -- & -- & -- & -- & -. & $<.005$ & $<.005$ \\
\hline 195 & $10-15-90$ & -- & -. & -- & -- & -- & -- & -. & $<.005$ & $<.005$ \\
\hline 196 & $10-17-90$ & -- & - & -. & .. & -. & -. & -- & $<.005$ & $<.005$ \\
\hline 197 & $10-16-90$ & - & -- &.- & -. & -- &.- & -- & $<.005$ & $<.005$ \\
\hline 198 & $10-17-90$ & -- & -- & -. & -. & - & -. & -- & $<.005$ & $<.005$ \\
\hline 199 & $10-17-90$ & -- & -. & .. & -. & -- & -. & -- & $<.005$ & $<.005$ \\
\hline 200 & $10-18-90$ & -- & -- & -- & -- & -- & -. & -- & $<.005$ & $<.005$ \\
\hline
\end{tabular}


Table 2. Water-level and water-quality measurements and nearby soil-gas-methane concentrations for selected water wells and springs--Continued

\begin{tabular}{|c|c|c|c|c|c|c|c|c|c|c|}
\hline $\begin{array}{l}\text { Num- } \\
\text { ber } \\
\text { on } \\
\text { plate } \\
1\end{array}$ & $\begin{array}{c}\text { Date } \\
\text { sampled }\end{array}$ & $\begin{array}{l}\text { Potas- } \\
\text { sium, } \\
\text { dis- } \\
\text { solved } \\
\text { (mg/L as } \\
\mathrm{K} \text { ) }\end{array}$ & $\begin{array}{c}\text { Sulfate, } \\
\text { dls- } \\
\text { solved } \\
\text { (mg/L as } \\
\left.\mathrm{SO}_{4}\right)\end{array}$ & $\begin{array}{l}\text { Chloride, } \\
\text { dis- } \\
\text { solved } \\
\text { (mg/L as } \\
\text { Cl) }\end{array}$ & $\begin{array}{l}\text { Bromide, } \\
\text { dls- } \\
\text { solved } \\
\text { (mg/L as } \\
\mathrm{Br} \text { ) }\end{array}$ & $\begin{array}{l}\text { SIllca, } \\
\text { dis- } \\
\text { solved } \\
(\mathrm{mg} / \mathrm{L} \text { as } \\
\left.\mathrm{SiO}_{2}\right)\end{array}$ & $\begin{array}{l}\text { Iron, } \\
\text { dls- } \\
\text { solved } \\
(\mu \mathrm{g} / \mathrm{L} \text { as } \\
\mathrm{Fe})\end{array}$ & $\begin{array}{c}\text { Manga- } \\
\text { nese, } \\
\text { dis- } \\
\text { solved } \\
(\mu \mathrm{g} / \mathrm{L} \text { as } \\
\text { Mn) }\end{array}$ & $\begin{array}{l}\text { Methane, } \\
\text { dis- } \\
\text { solved } \\
\text { (mg/L) }\end{array}$ & $\begin{array}{c}\text { Methane, } \\
\text { in } \\
\text { soil gas } \\
\left.\text { (mg/L } \mathrm{L}_{\mathrm{g}}\right)\end{array}$ \\
\hline 201 & $10-16-90$ & $\overline{--}$ & $\overline{--}$ & -- & -- & $\overline{--}$ & $\overline{--}$ & $\overline{--}$ & $<0.005$ & $<0.005$ \\
\hline 202 & $10-17-90$ & -- & -- & -. & -- & -- & -- & -- & $<.005$ & $<.005$ \\
\hline 203 & $10-17-90$ & 4.0 & 420 & 31 & .07 & 14 & 2,100 & 2,800 & .077 & $<.005$ \\
\hline 204 & $10-17-90$ & - & -- & -- & -- & -- & -- & -- & $<.005$ & $<.005$ \\
\hline 205 & $10-16-90$ & -- & -- & -- & -- & -- & -- & -- & $<.005$ & $<.005$ \\
\hline
\end{tabular}


Table 3. Gas-well records and maximum methane concentrations measured in soil gas adjacent to gas-well casings

[ft, feet; $\mathrm{mg} / \mathrm{L}_{\mathrm{g}}$, milligrams per liter of gas; GAS, producing or shut-in single-completion gas well; DA, hole drilled and abandoned; 2GAS, producing or shut-in dual-completion gas well; PA, gas well plugged and abandoned; WOC, gas well waiting on completion when sampled; FL, Fruitland Formation coals; PC, Pictured Cliffs Sandstone; LS, Lewis Shale; MV. Mesaverde Group; DK, Dakota Sandstone; --, producing formation not applicable; <, less than]

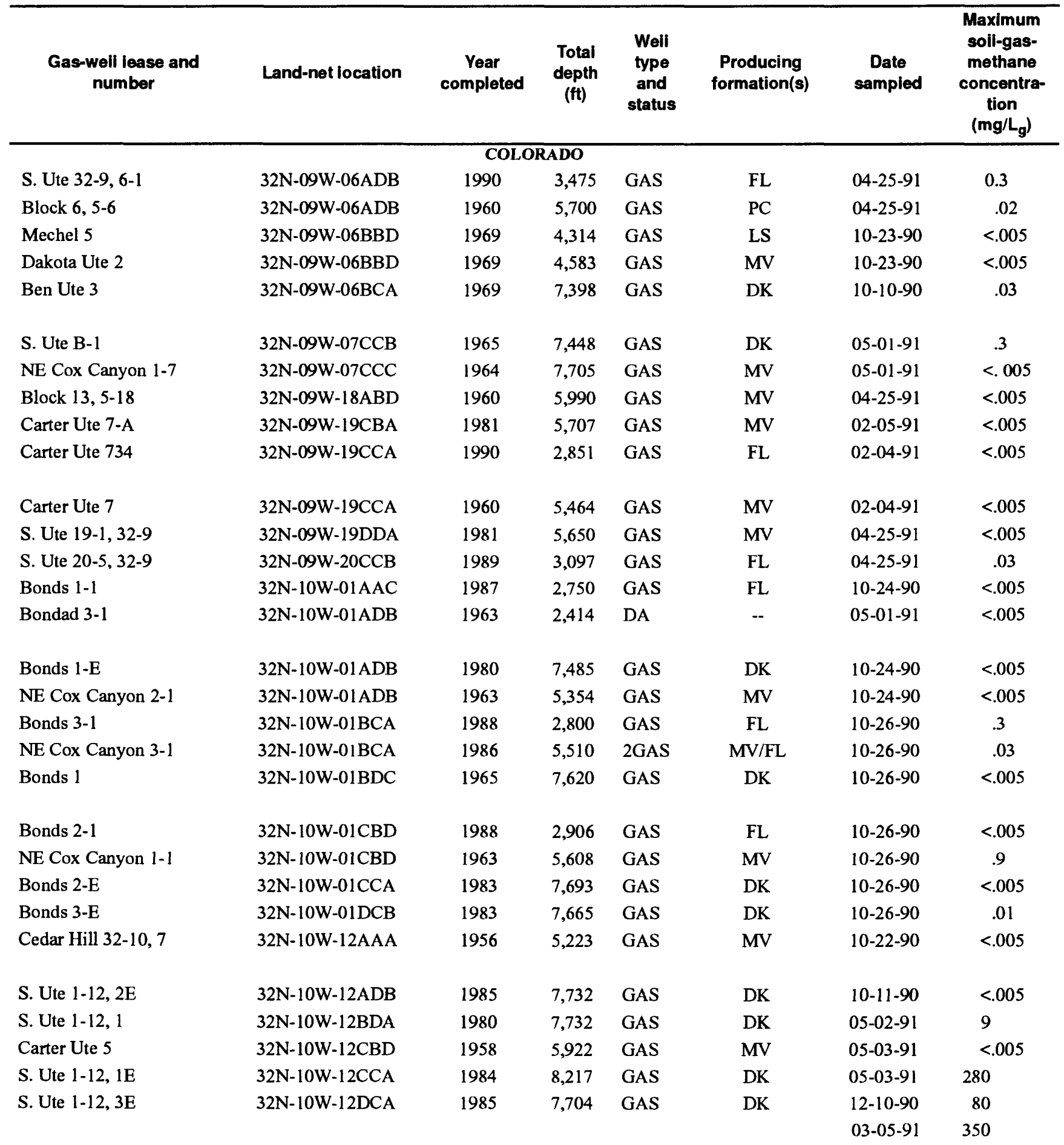


Table 3. Gas-well records and maximum methane concentrations measured in soil gas adjacent to gas-well casings--Continued

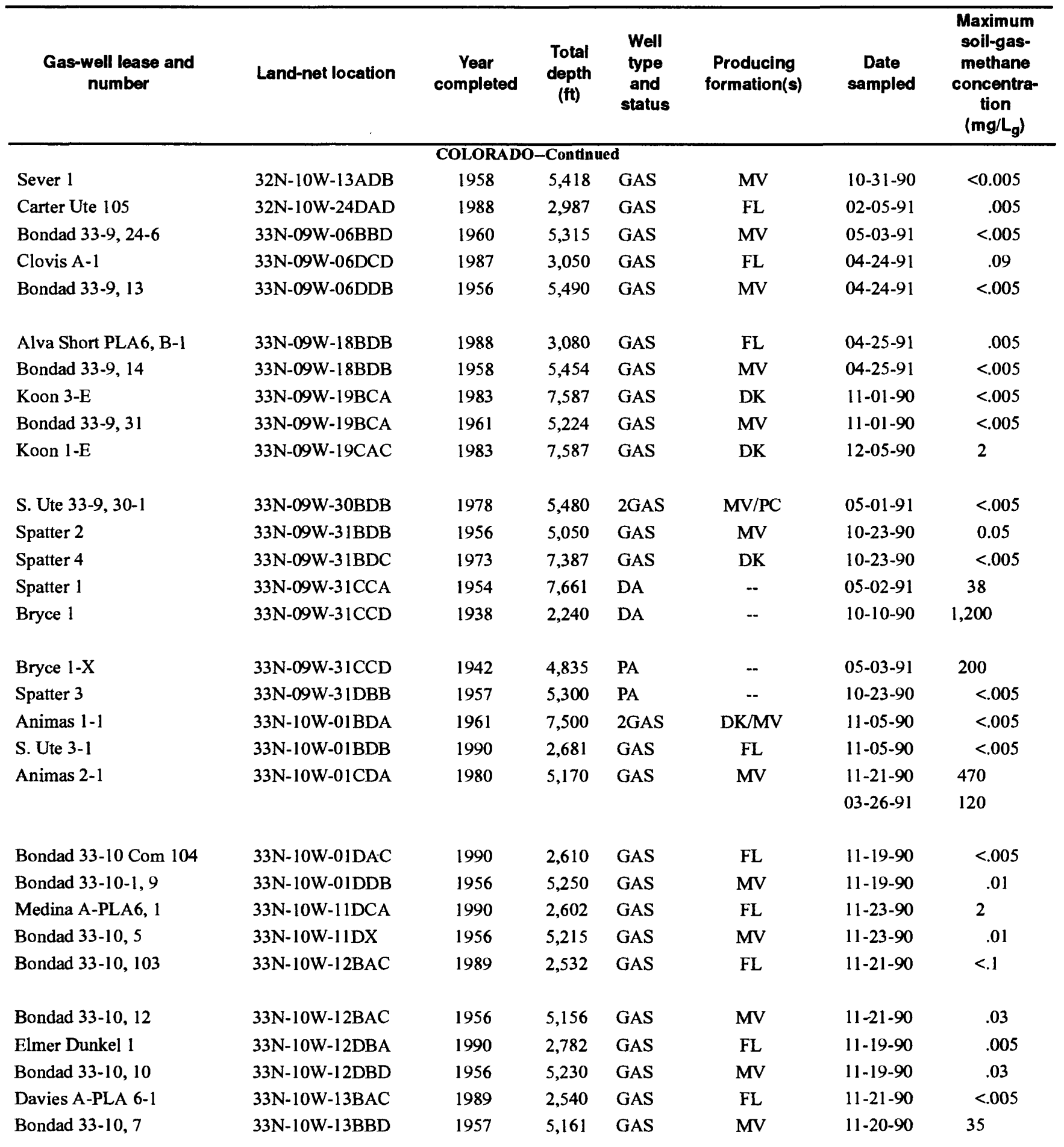


Table 3. Gas-well records and maximum methane concentrations measured in soil gas adjacent to gas-well casings--Continued

\begin{tabular}{|c|c|c|c|c|c|c|c|}
\hline $\begin{array}{c}\text { Gas-well lease and } \\
\text { number }\end{array}$ & Land-net location & $\begin{array}{c}\text { Year } \\
\text { completed }\end{array}$ & $\begin{array}{l}\text { Total } \\
\text { depth } \\
\text { (ft) }\end{array}$ & $\begin{array}{l}\text { Well } \\
\text { type } \\
\text { and } \\
\text { status }\end{array}$ & $\begin{array}{l}\text { Producing } \\
\text { formation(s) }\end{array}$ & $\begin{array}{c}\text { Date } \\
\text { sampled }\end{array}$ & $\begin{array}{c}\text { Maximum } \\
\text { soil-gas- } \\
\text { methane } \\
\text { concentra- } \\
\text { tlon } \\
\left(\mathrm{mg} / \mathrm{L}_{\mathrm{g}}\right)\end{array}$ \\
\hline \multicolumn{8}{|c|}{ COLORADO-Continued } \\
\hline Ute B-1 & $33 \mathrm{~N}-10 \mathrm{~W}-13 \mathrm{DBD}$ & 1961 & 5,252 & GAS & MV & $11-01-90$ & 0.01 \\
\hline Bondad 33-10, 17 & $33 \mathrm{~N}-10 \mathrm{~W}-14 \mathrm{DBD}$ & 1960 & 5,294 & GAS & MV & $11-23-90$ & $<.005$ \\
\hline S. Ute $33-10,23-2$ & $33 \mathrm{~N}-10 \mathrm{~W}-23 \mathrm{AAC}$ & 1980 & 5,350 & GAS & MV & $11-23-90$ & 2 \\
\hline Craig 1-E & $33 \mathrm{~N}-10 \mathrm{~W}-24 \mathrm{AAC}$ & 1984 & 7,600 & GAS & DK & $11-01-90$ & 14 \\
\hline Ute $C-2$ & $33 \mathrm{~N}-10 \mathrm{~W}-24 \mathrm{AAC}$ & 1984 & 5,350 & GAS & MV & $11-01-90$ & 25 \\
\hline S. Ute $33-10,24-3$ & $33 \mathrm{~N}-10 \mathrm{~W}-24 \mathrm{BAC}$ & 1990 & 2,605 & GAS & FL & $10-31-90$ & 5 \\
\hline S. Ute $33-10,24-1$ & $33 \mathrm{~N}-10 \mathrm{~W}-24 \mathrm{BAC}$ & 1964 & 5,311 & GAS & MV & $11-21-90$ & $<.005$ \\
\hline Craig 2-E & $33 \mathrm{~N}-10 \mathrm{~W}-24 \mathrm{BDB}$ & 1984 & 7,657 & GAS & DK & $11-21-90$ & .005 \\
\hline Craig 3-E & $33 \mathrm{~N}-10 \mathrm{~W}-24 \mathrm{CBD}$ & 1985 & 7,875 & GAS & DK & $10-30-90$ & 21 \\
\hline S. Ute $33-10,24-2$ & $33 \mathrm{~N}-10 \mathrm{~W}-24 \mathrm{CDB}$ & 1980 & 5,443 & GAS & MV & $10-30-90$ & .005 \\
\hline Craig 1 & $33 \mathrm{~N}-10 \mathrm{~W}-24 \mathrm{DBC}$ & 1975 & 7,585 & GAS & DK & $10-31-90$ & 65 \\
\hline Ute C-1 & $33 \mathrm{~N}-10 \mathrm{~W}-24 \mathrm{DBD}$ & 1963 & 5,260 & GAS & MV & $10-31-90$ & $<.005$ \\
\hline Sharp 3-E & $33 \mathrm{~N}-10 \mathrm{~W}-25 \mathrm{AAD}$ & 1983 & 7,559 & GAS & DK & $10-31-90$ & .01 \\
\hline Bondad 3̦3-10, 1-25 & $33 \mathrm{~N}-10 \mathrm{~W}-25 \mathrm{ABD}$ & 1955 & 2,392 & DA & -- & $05-02-91$ & $<.005$ \\
\hline \multirow[t]{2}{*}{ Bondad 33-10, 3} & $33 \mathrm{~N}-10 \mathrm{~W}-25 \mathrm{ABD}$ & 1955 & 5,199 & GAS & MV & $10-31-90$ & 430 \\
\hline & & & & & & $03-05-91$ & 220 \\
\hline Sharp 1-E & $33 \mathrm{~N}-10 \mathrm{~W}-25 \mathrm{BAC}$ & 1980 & 7,651 & GAS & DK & $10-30-90$ & .005 \\
\hline Sharp 2-E & $33 \mathrm{~N}-10 \mathrm{~W}-25 \mathrm{CAC}$ & 1983 & 7,623 & GAS & DK & $10-30-90$ & .1 \\
\hline Sharp 1 & $33 \mathrm{~N}-10 \mathrm{~W}-25 \mathrm{DBC}$ & 1972 & 7,518 & GAS & DK & $10-30-90$ & $<.005$ \\
\hline M.F. Sharp 1 & $33 \mathrm{~N}-10 \mathrm{~W}-25 \mathrm{DDB}$ & 1990 & 2,595 & GAS & FL & $10-30-90$ & $<.005$ \\
\hline S. Ute A-3 & $33 \mathrm{~N}-10 \mathrm{~W}-26 \mathrm{DAC}$ & 1965 & 7,662 & GAS & DK & $10-29-90$ & $<.005$ \\
\hline S. Ute $1-26$ & $33 \mathrm{~N}-10 \mathrm{~W}-26 \mathrm{DAC}$ & 1957 & 5,382 & GAS & MV & $10-29-90$ & $<.005$ \\
\hline S. Ute A-2 & 33N-10W-35DAB & 1965 & 7,845 & GAS & DK & $11-23-90$ & $<.005$ \\
\hline Bondad Ute 1-36 & 33N-10W-36BBD & 1989 & 3,300 & GAS & FL & $11-23-90$ & 2 \\
\hline Bondad 33-10, 6 & 33N-10W-36BDB & 1956 & 5,318 & GAS & MV & $10-29-90$ & $<.005$ \\
\hline Bondad 33-10, 24 & $33 \mathrm{~N}-10 \mathrm{~W}-36 \mathrm{DAC}$ & 1966 & 7,418 & GAS & DK & $10-10-90$ & 320 \\
\hline Bondad 33-10, 18 & $33 \mathrm{~N}-10 \mathrm{~W}-36 \mathrm{DBD}$ & 1957 & 5,190 & GAS & MV & $10-25-90$ & $<.005$ \\
\hline Wheeler $7 \mathrm{U}-1$ & $34 \mathrm{~N}-09 \mathrm{~W}-07 \mathrm{CAC}$ & 1986 & 2,621 & GAS & FL & $04-23-91$ & 25 \\
\hline Koshak B-1 & 34N-09W-08BDB & 1989 & 2,740 & GAS & FL & $11-05-90$ & 14 \\
\hline Koshak A-1 & $34 \mathrm{~N}-09 \mathrm{~W}-08 \mathrm{DBC}$ & 1988 & 2,555 & GAS & FL & $11-05-90$ & .02 \\
\hline Isgar $\mathrm{A}-1$ & 34N-09W-1 8BDA & 1989 & 2,595 & GAS & FL & $04-25-91$ & .07 \\
\hline
\end{tabular}


Table 3. Gas-well records and maximum methane concentrations measured in soil gas adjacent to gas-well casings--Continued

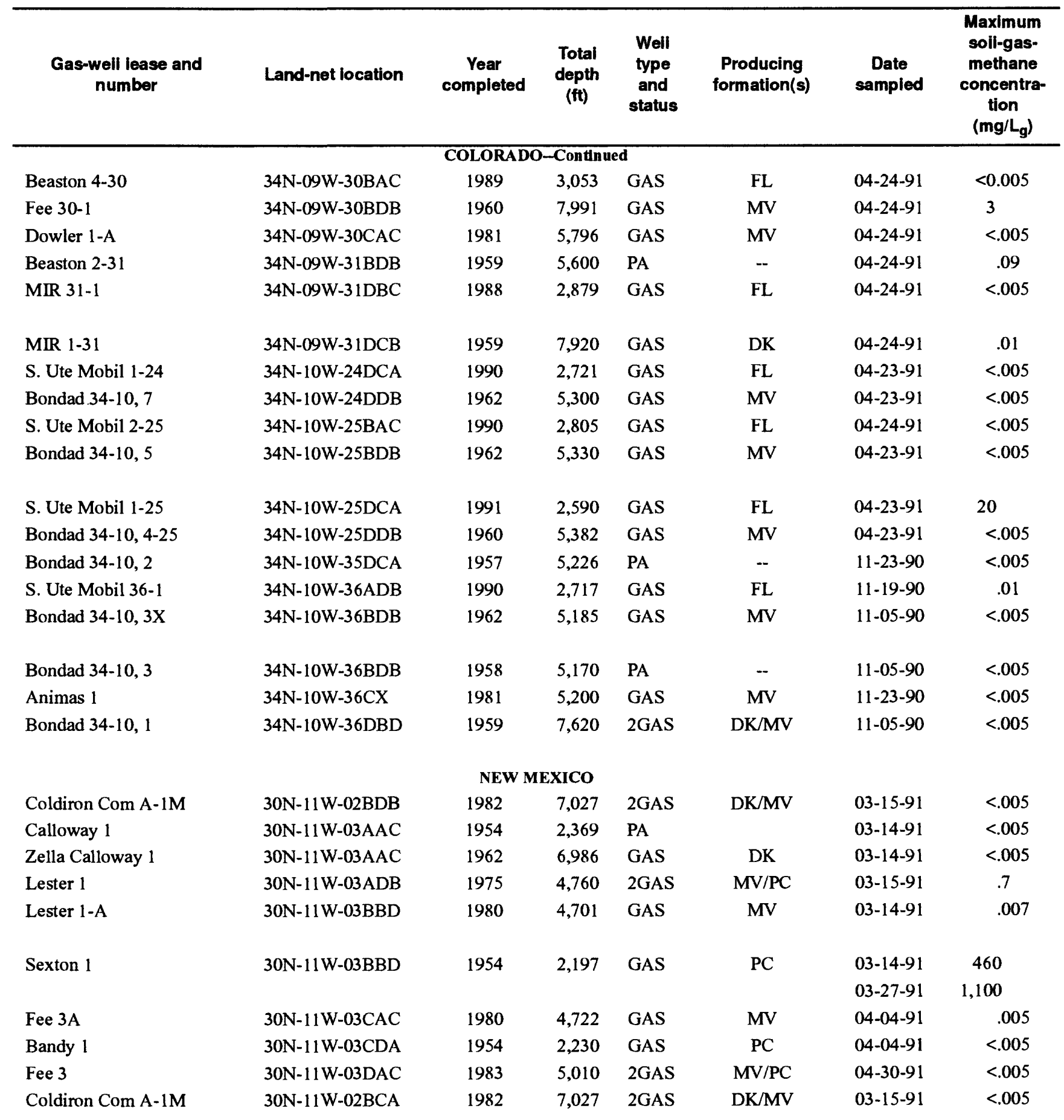


Table 3. Gas-well records and maximum methane concentrations measured in soil gas adjacent to gas-well casings--Continued

\begin{tabular}{|c|c|c|c|c|c|c|c|}
\hline $\begin{array}{c}\text { Gas-well lease and } \\
\text { number }\end{array}$ & Land-net iocation & $\begin{array}{c}\text { Year } \\
\text { completed }\end{array}$ & $\begin{array}{l}\text { Totai } \\
\text { depth } \\
\text { (ft) }\end{array}$ & $\begin{array}{l}\text { Well } \\
\text { type } \\
\text { and } \\
\text { status }\end{array}$ & $\begin{array}{l}\text { Producing } \\
\text { formation(s) }\end{array}$ & $\begin{array}{l}\text { Date } \\
\text { sampled }\end{array}$ & $\begin{array}{c}\text { Maximum } \\
\text { soil-gas- } \\
\text { methane } \\
\text { concentra- } \\
\text { tion } \\
\left(\mathrm{mg} / \mathrm{L}_{\mathrm{g}}\right)\end{array}$ \\
\hline \multicolumn{8}{|c|}{ NEW MEXICO-Continued } \\
\hline Hampton 2 & $30 \mathrm{~N}-11 \mathrm{~W}-03 \mathrm{DCA}$ & 1953 & 2,285 & PA & -- & $04-04-91$ & 0.006 \\
\hline Haynie 2 & $30 \mathrm{~N}-11 \mathrm{~W}-04 \mathrm{ABD}$ & 1980 & 6,860 & 2GAS & DK/MV & $03-25-91$ & $<.005$ \\
\hline Haynie 1 & $30 \mathrm{~N}-11 \mathrm{~W}-04 \mathrm{ACA}$ & 1954 & 2,238 & PA & -- & $04-01-91$ & $<.005$ \\
\hline Bobbie Herrera 1 & $30 \mathrm{~N}-11 \mathrm{~W}-04 \mathrm{CAC}$ & 1979 & 2,350 & 2GAS & $\mathrm{PC} / \mathrm{FL}$ & $04-01-91$ & $<.005$ \\
\hline Fee 4 & $30 \mathrm{~N}-11 \mathrm{~W}-04 \mathrm{DAC}$ & 1980 & 4,863 & GAS & MV & $04-04-91$ & $<.005$ \\
\hline Ona Magee 1 & $30 \mathrm{~N}-11 \mathrm{~W}-04 \mathrm{DDB}$ & 1961 & 6,778 & GAS & DK & $04-04-91$ & .1 \\
\hline Roberts 1 & $30 \mathrm{~N}-11 \mathrm{~W}-04 \mathrm{DDB}$ & 1954 & 2,167 & GAS & $\mathrm{PC}$ & $04-30-91$ & 21 \\
\hline Thomas 1 & $30 \mathrm{~N}-11 \mathrm{~W}-09 \mathrm{AAC}$ & 1953 & 2,154 & GAS & PC & $04-01-91$ & $<.005$ \\
\hline Fee 9-A & $30 \mathrm{~N}-11 \mathrm{~W}-09 \mathrm{ABD}$ & 1981 & 4,812 & GAS & MV & $04-04-91$ & $<.005$ \\
\hline Hampton D-1 & $30 \mathrm{~N}-11 \mathrm{~W}-10 \mathrm{ABD}$ & 1962 & 6,906 & GAS & DK & $04-05-91$ & $<.005$ \\
\hline Hampton 3 & $30 \mathrm{~N}-11 \mathrm{~W}-10 \mathrm{ABD}$ & 1956 & 4,757 & GAS & MV & $04-05-91$ & $<.005$ \\
\hline Hampton 3-A & $30 \mathrm{~N}-11 \mathrm{~W}-10 \mathrm{BBD}$ & 1977 & 4,842 & GAS & MV & $04-04-91$ & $<.005$ \\
\hline Scott 7 & $31 \mathrm{~N}-10 \mathrm{~W}-03 \mathrm{ABC}$ & 1955 & 5,365 & GAS & MV & $12-14-90$ & .06 \\
\hline Scott 12 & $31 \mathrm{~N}-10 \mathrm{~W}-03 \mathrm{ACA}$ & 1975 & 2,979 & GAS & $\mathrm{PC}$ & $12-13-90$ & .02 \\
\hline Scott 13 & $31 \mathrm{~N}-10 \mathrm{~W}-03 \mathrm{BDB}$ & 1975 & 2,904 & GAS & $\mathrm{PC}$ & $12-14-90$ & 16 \\
\hline Scott 7-A & $31 \mathrm{~N}-10 \mathrm{~W}-03 \mathrm{BCD}$ & 1976 & 5,425 & GAS & MV & $\begin{array}{l}12-13-90 \\
03-27-91\end{array}$ & $\begin{array}{c}.9^{\mathrm{a}} \\
\text { no data }\end{array}$ \\
\hline Lucerne A-6 & $31 \mathrm{~N}-10 \mathrm{~W}-03 \mathrm{CCA}$ & 1978 & 3,255 & PA & -- & $12-12-90$ & 130 \\
\hline Lucerne A-3 & $31 \mathrm{~N}-10 \mathrm{~W}-03 \mathrm{CCA}$ & 1955 & 5,656 & GAS & MV & $12-12-90$ & 49 \\
\hline Wood Com A-1 & $31 \mathrm{~N}-10 \mathrm{~W}-04 \mathrm{ABD}$ & 1984 & 2,694 & GAS & FL & $12-05-90$ & .4 \\
\hline Usselman Com 1 & $31 \mathrm{~N}-10 \mathrm{~W}-04 \mathrm{ABD}$ & 1954 & 5,075 & GAS & MV & $12-05-90$ & .008 \\
\hline Usselman Com B-1 & $31 \mathrm{~N}-10 \mathrm{~W}-04 \mathrm{ACA}$ & 1977 & 2,970 & GAS & PC & $12-07-90$ & .005 \\
\hline Usselman Com 1-A & $31 N-10 W-04 B C D$ & 1978 & 5,182 & GAS & MV & $12-04-90$ & $<.005$ \\
\hline Usselman Com C-1 & $31 \mathrm{~N}-10 \mathrm{~W}-04 \mathrm{BCD}$ & 1978 & 2,984 & GAS & PC & $12-04-90$ & $<.005$ \\
\hline Scott 6 & $31 \mathrm{~N}-10 \mathrm{~W}-04 \mathrm{CAC}$ & 1954 & 5,417 & GAS & MV & $12-11-90$ & $<.005$ \\
\hline Scott 10 & $31 \mathrm{~N}-10 \mathrm{~W}-04 \mathrm{CCA}$ & 1975 & 2,957 & GAS & $\mathrm{PC}$ & $12-11-90$ & $<.005$ \\
\hline \multirow[t]{2}{*}{ Scott 6-A } & $31 \mathrm{~N}-10 \mathrm{~W}-04 \mathrm{DBD}$ & 1977 & 5,535 & GAS & MV & $12-11-90$ & 97 \\
\hline & & & & & & $04-15-91$ & 370 \\
\hline Lucerne A-10 & $31 \mathrm{~N}-10 \mathrm{~W}-04 \mathrm{DDB}$ & 1978 & 3,115 & GAS & PC & $12-13-90$ & $<.005$ \\
\hline Marcotte Com C-1 & $31 \mathrm{~N}-10 \mathrm{~W}-05 \mathrm{ABD}$ & 1979 & 2,854 & GAS & $\mathrm{PC}$ & $04-30-91$ & $<.005$ \\
\hline Marcotte Com 1 & $31 \mathrm{~N}-10 \mathrm{~W}-05 \mathrm{ADB}$ & 1953 & 5,160 & GAS & MV & $01-17-91$ & 2 \\
\hline McEwen Com 1-A & $31 \mathrm{~N}-10 \mathrm{~W}-05 \mathrm{BBC}$ & 1976 & 5,400 & GAS & MV & $01-24-91$ & $<.005$ \\
\hline
\end{tabular}


Table 3. Gas-well records and maximum methane concentrations measured in soil gas adjacent to gas-well casings--Continued

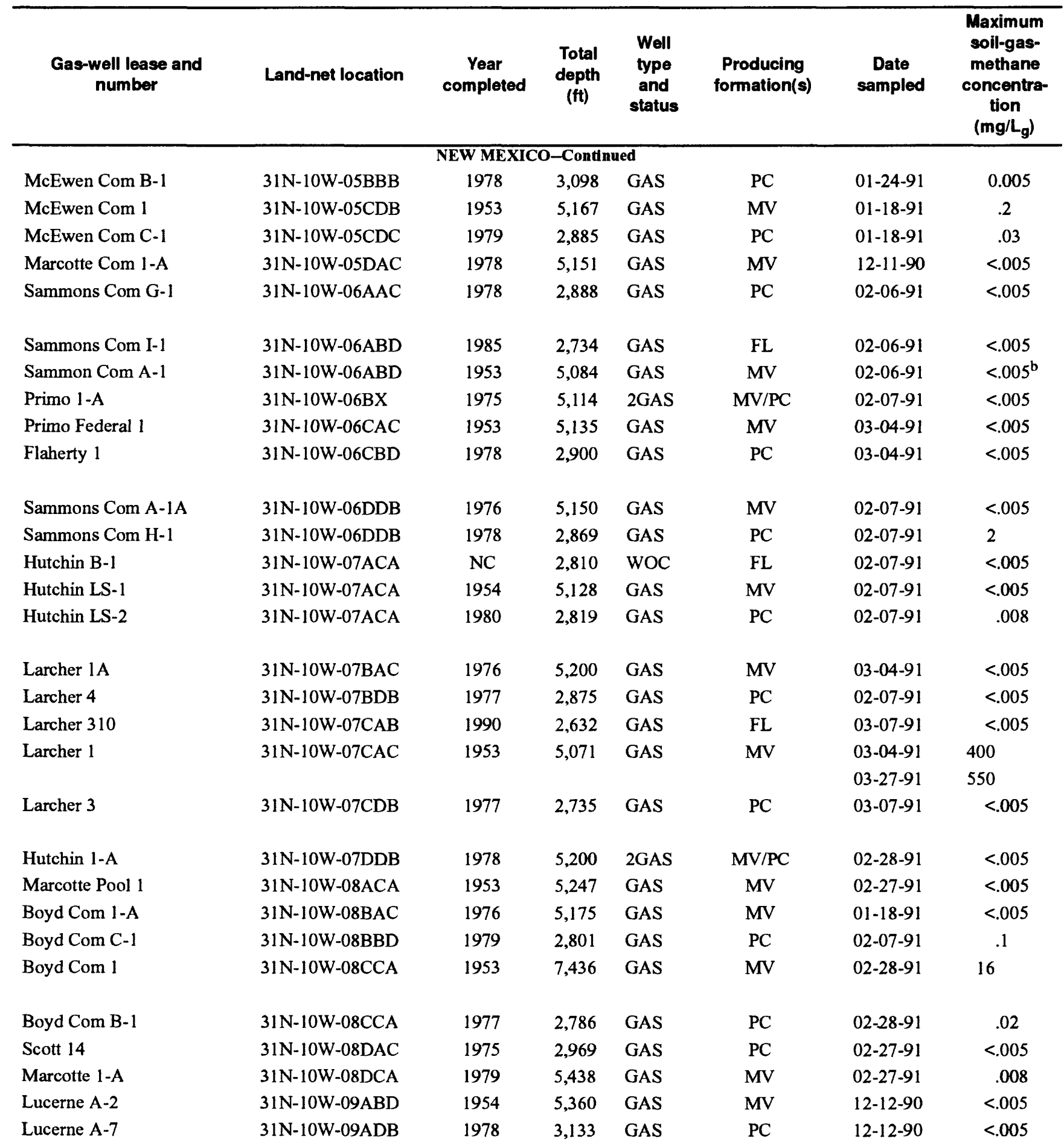


Table 3. Gas-well records and maximum methane concentrations measured in soil gas adjacent to gas-well casings--Continued

\begin{tabular}{|c|c|c|c|c|c|c|c|}
\hline $\begin{array}{c}\text { Gas-weil lease and } \\
\text { number }\end{array}$ & Land-net location & $\begin{array}{c}\text { Year } \\
\text { completed }\end{array}$ & $\begin{array}{l}\text { Total } \\
\text { depth } \\
\text { (ft) }\end{array}$ & $\begin{array}{l}\text { Well } \\
\text { type } \\
\text { and } \\
\text { status }\end{array}$ & $\begin{array}{l}\text { Producing } \\
\text { formation(s) }\end{array}$ & $\begin{array}{c}\text { Date } \\
\text { sampled }\end{array}$ & $\begin{array}{l}\text { Maximum } \\
\text { soil-gas- } \\
\text { methane } \\
\text { concentra- } \\
\text { tion } \\
\text { (mg/ } / \mathrm{g})\end{array}$ \\
\hline \multicolumn{8}{|c|}{ NEW MEXICO-Continued } \\
\hline Lucerne A-1 A & $31 N-10 W-09 B A C$ & 1979 & 5,488 & GAS & MV & $12-11-90$ & $<0.005$ \\
\hline Scott 11 & $31 \mathrm{~N}-10 \mathrm{~W}-09 \mathrm{BAC}$ & 1975 & 2,996 & GAS & $\mathrm{PC}$ & $12-11-90$ & .008 \\
\hline Lucerne A-1 & $31 \mathrm{~N}-10 \mathrm{~W}-09 \mathrm{CBD}$ & 1954 & 5,353 & GAS & MV & $02-27-90$ & $<.005$ \\
\hline Lucerne A-4A & $31 \mathrm{~N}-10 \mathrm{~W}-10 \mathrm{BAC}$ & 1978 & 5,668 & 2GAS & $\mathrm{MV} / \mathrm{PC}$ & $12-12-90$ & $<.005$ \\
\hline Scott 22 & $31 \mathrm{~N}-10 \mathrm{~W}-17 \mathrm{AAC}$ & 1976 & 2,961 & GAS & PC & $02-28-91$ & .3 \\
\hline Scott 4 & $31 \mathrm{~N}-10 \mathrm{~W}-17 \mathrm{ADB}$ & 1952 & 5,341 & GAS & MV & $02-28-91$ & $<.005$ \\
\hline Scott 9 & $31 \mathrm{~N}-10 \mathrm{~W}-17 \mathrm{BAC}$ & 1975 & 2,968 & GAS & $\mathrm{PC}$ & $02-28-91$ & $<.005$ \\
\hline Hudson 5-A & $31 \mathrm{~N}-10 \mathrm{~W}-17 \mathrm{BDB}$ & 1977 & 5,427 & GAS & MV & $03-07-91$ & .009 \\
\hline McCoy Com A-1 & $31 \mathrm{~N}-10 \mathrm{~W}-18 \mathrm{ADB}$ & 1952 & 5,005 & GAS & MV & $02-19-91$ & $<.005$ \\
\hline McCoy Com A-1A & $31 \mathrm{~N}-10 \mathrm{~W}-18 \mathrm{BDB}$ & 1977 & 4,988 & GAS & PC & $02-11-91$ & 180 \\
\hline McCoy B-1 & $31 \mathrm{~N}-10 \mathrm{~W}-18 \mathrm{BDB}$ & 1983 & 2,640 & GAS & $\mathrm{PC}$ & $02-11-91$ & .3 \\
\hline Caneple Com C-1 & $31 \mathrm{~N}-10 \mathrm{~W}-18 \mathrm{CAC}$ & 1972 & 2,584 & GAS & PC & $02-11-91$ & $<.005$ \\
\hline Caneple Com 1 & $31 \mathrm{~N}-10 \mathrm{~W}-18 \mathrm{CDB}$ & 1954 & 4,950 & GAS & MV & $02-11-91$ & $<.005$ \\
\hline Crandell 7 & $31 \mathrm{~N}-10 \mathrm{~W}-18 \mathrm{DAC}$ & 1978 & 2,802 & PA & -- & $02-19-91$ & $<.005$ \\
\hline Caneple Com 1-A & $31 \mathrm{~N}-10 \mathrm{~W}-18 \mathrm{DAC}$ & 1976 & 5,200 & GAS & MV & $02-19-91$ & $<.005$ \\
\hline Crandell l & $31 \mathrm{~N}-10 \mathrm{~W}-19 \mathrm{AAC}$ & 1951 & 5,246 & GAS & MV & $02-19-91$ & $<.005$ \\
\hline Crandell 4 & $31 \mathrm{~N}-10 \mathrm{~W}-19 \mathrm{AAC}$ & 1969 & 2,775 & GAS & $\mathrm{PC}$ & $02-19-91$ & .2 \\
\hline Crandall 2-A & $31 \mathrm{~N}-10 \mathrm{~W}-19 \mathrm{BAC}$ & 1976 & 5,218 & GAS & MV & $02-19-91$ & .3 \\
\hline Crandell 6 & $31 \mathrm{~N}-10 \mathrm{~W}-19 \mathrm{BAC}$ & 1969 & 2,696 & GAS & PC & $02-19-91$ & 560 \\
\hline Crandell 2 & $31 \mathrm{~N}-10 \mathrm{~W}-19 \mathrm{CCA}$ & 1953 & 5,070 & GAS & MV & $02-15-91$ & 460 \\
\hline Crandell 5 & $31 \mathrm{~N}-10 \mathrm{~W}-19 \mathrm{CCA}$ & 1969 & 2,714 & GAS & $\mathrm{PC}$ & $02-15-91$ & 240 \\
\hline Pierce 2-A & $31 \mathrm{~N}-10 \mathrm{~W}-30 \mathrm{BCA}$ & 1977 & 5,343 & GAS & MV & $02-13-91$ & $<.005$ \\
\hline Pierce 5 & $31 \mathrm{~N}-10 \mathrm{~W}-30 \mathrm{BCA}$ & 1969 & 2,730 & GAS & $\mathrm{PC}$ & $02-13-91$ & .04 \\
\hline Mudge LS-8 & $31 \mathrm{~N}-11 \mathrm{~W}-12 \mathrm{AAC}$ & 1954 & 5,250 & GAS & MV & $03-07-91$ & $<.005$ \\
\hline Mudge LS-8A & $31 \mathrm{~N}-11 \mathrm{~W}-12 \mathrm{DAC}$ & 1979 & 5,325 & GAS & MV & $03-07-91$ & $<.005$ \\
\hline Ridenour Com A-1 & $31 \mathrm{~N}-11 \mathrm{~W}-13 \mathrm{ACA}$ & 1989 & 2,772 & GAS & FL & $03-07-91$ & $<.005$ \\
\hline Larcher 2 & $31 \mathrm{~N}-11 \mathrm{~W}-13 \mathrm{ACA}$ & 1976 & 2,705 & GAS & $\mathrm{PC}$ & $03-12-91$ & $<.005$ \\
\hline Ridenour Com 1 & $31 \mathrm{~N}-11 \mathrm{~W}-13 \mathrm{ADB}$ & 1954 & 5,057 & GAS & MV & $03-07-91$ & $<.005$ \\
\hline Randleman 3 & $31 \mathrm{~N}-11 \mathrm{~W}-13 \mathrm{BBD}$ & 1970 & 2,578 & GAS & PC & $03-12-91$ & $<.005$ \\
\hline Randleman 1-A & $31 \mathrm{~N}-11 \mathrm{~W}-13 \mathrm{BCA}$ & 1976 & 5,063 & GAS & MV & $03-12-91$ & $<.005$ \\
\hline
\end{tabular}


Table 3. Gas-well records and maximum methane concentrations measured in soil gas adjacent to gas-well casings--Continued

\begin{tabular}{|c|c|c|c|c|c|c|c|}
\hline $\begin{array}{c}\text { Gas-well lease and } \\
\text { number }\end{array}$ & Land-net location & $\begin{array}{c}\text { Year } \\
\text { completed }\end{array}$ & $\begin{array}{c}\text { Total } \\
\text { depth } \\
\text { (ft) }\end{array}$ & $\begin{array}{l}\text { Well } \\
\text { typo } \\
\text { and } \\
\text { status }\end{array}$ & $\begin{array}{l}\text { Producing } \\
\text { formation(s) }\end{array}$ & $\begin{array}{l}\text { Date } \\
\text { sampled }\end{array}$ & $\begin{array}{l}\text { Maximum } \\
\text { soil-gas- } \\
\text { methane } \\
\text { concentra- } \\
\text { tion } \\
\left(\mathrm{mg} / \mathrm{L}_{\mathrm{g}}\right)\end{array}$ \\
\hline \multicolumn{8}{|c|}{ NEW MEXICO-Continued } \\
\hline Randleman 1 & $31 \mathrm{~N}-11 \mathrm{~W}-13 \mathrm{CAC}$ & 1951 & 4,980 & GAS & MV & $03-12-91$ & $<0.005$ \\
\hline Randleman 2 & $31 \mathrm{~N}-11 \mathrm{~W}-13 \mathrm{CCA}$ & 1970 & 2,633 & PA & -- & $05-01-91$ & $<.005$ \\
\hline Ridenour Com 1-A & $31 \mathrm{~N}-11 \mathrm{~W}-13 \mathrm{DDB}$ & 1977 & 4,950 & GAS & MV & $02-11-91$ & $<.005$ \\
\hline Canepele Com B-1 & $31 \mathrm{~N}-11 \mathrm{~W}-13 \mathrm{DDB}$ & 1971 & 2,569 & GAS & PC & $02-11-91$ & $<.005$ \\
\hline Neil LS- IA & $31 \mathrm{~N}-11 \mathrm{~W}-14 \mathrm{DBD}$ & 1957 & 5,011 & GAS & MV & $03-12-91$ & 100 \\
\hline Neal Com 2-E & $31 \mathrm{~N}-11 \mathrm{~W}-14 \mathrm{DCA}$ & 1984 & 7,160 & GAS & DK & $03-12-91$ & $<.005$ \\
\hline Neil LS-10 & $31 \mathrm{~N}-11 \mathrm{~W}-14 \mathrm{DDB}$ & 1972 & 2,716 & GAS & $\mathrm{PC}$ & $03-12-91$ & $<.005$ \\
\hline Nye LS-1 & $31 \mathrm{~N}-11 \mathrm{~W}-23 \mathrm{AAC}$ & 1953 & 5,062 & GAS & MV & $03-12-91$ & .02 \\
\hline Mudge LS-31 & $31 \mathrm{~N}-11 \mathrm{~W}-23 \mathrm{AAC}$ & 1972 & 2,596 & GAS & $\mathrm{PC}$ & $03-12-91$ & $<.005$ \\
\hline Mudge LS-7A & $31 \mathrm{~N}-11 \mathrm{~W}-23 \mathrm{BDB}$ & 1978 & 5,182 & GAS & MV & $03-13-91$ & $<.005$ \\
\hline Mudge LS-50 & $31 \mathrm{~N}-11 \mathrm{~W}-23 \mathrm{BDB}$ & 1981 & 2,599 & GAS & $\mathrm{PC}$ & $03-13-91$ & $<.005$ \\
\hline Mudge LS-37 & $31 \mathrm{~N}-11 \mathrm{~W}-23 \mathrm{CAC}$ & 1972 & 2,567 & GAS & $\mathrm{PC}$ & $03-13-91$ & $<.005$ \\
\hline Mudge LS-7 & $31 \mathrm{~N}-11 \mathrm{~W}-23 \mathrm{CCA}$ & 1955 & 4,945 & GAS & MV & $03-13-91$ & $<.005$ \\
\hline Nye LS-1A & $31 \mathrm{~N}-11 \mathrm{~W}-23 \mathrm{DCA}$ & 1979 & 5,022 & GAS & MV & $02-11-91$ & 8 \\
\hline Mudge LS-32 & $31 \mathrm{~N}-11 \mathrm{~W}-23 \mathrm{DCA}$ & 1972 & 2,490 & GAS & $\mathrm{PC}$ & $02-11-91$ & 120 \\
\hline Jensen 1 & $31 \mathrm{~N}-11 \mathrm{~W}-24 \mathrm{AAC}$ & 1970 & 2,547 & GAS & $\mathrm{PC}$ & $02-08-91$ & $<.005$ \\
\hline Ruple 1-A & $31 \mathrm{~N}-11 \mathrm{~W}-24 \mathrm{ABD}$ & 1976 & 5,043 & GAS & MV & $02-08-91$ & $<.005$ \\
\hline Turner 1 & $31 \mathrm{~N}-11 \mathrm{~W}-24 \mathrm{BDC}$ & 1953 & 4,911 & GAS & MV & $02-08-91$ & 20 \\
\hline Turner 3 & $31 \mathrm{~N}-11 \mathrm{~W}-24 \mathrm{BDX}$ & 1970 & 2,494 & GAS & $\mathrm{PC}$ & $02-08-91$ & .01 \\
\hline Turner 1-A & $31 \mathrm{~N}-11 \mathrm{~W}-24 \mathrm{CCA}$ & 1976 & 4,950 & GAS & MV & $02-12-91$ & $<.005$ \\
\hline Turner 2 & $31 \mathrm{~N}-11 \mathrm{~W}-24 \mathrm{CDB}$ & 1969 & 2,574 & GAS & $\mathrm{PC}$ & $02-12-91$ & .6 \\
\hline Ruple 2 & $31 \mathrm{~N}-11 \mathrm{~W}-24 \mathrm{DCA}$ & 1970 & 2,546 & GAS & $\mathrm{PC}$ & $02-15-91$ & $<.005$ \\
\hline Ruple 1-X & $31 \mathrm{~N}-11 \mathrm{~W}-24 \mathrm{DCX}$ & 1951 & 4,951 & GAS & MV & $02-15-91$ & 140 \\
\hline Bruington 2 & $31 \mathrm{~N}-11 \mathrm{~W}-25 \mathrm{AAC}$ & 1969 & 2,647 & GAS & PC & $02-13-91$ & .005 \\
\hline Bruington 1 & $31 \mathrm{~N}-11 \mathrm{~W}-25 \mathrm{ACA}$ & 1953 & 4,969 & GAS & MV & $02-12-91$ & $<.005$ \\
\hline \multirow[t]{2}{*}{ Bruington 3} & $31 \mathrm{~N}-11 \mathrm{~W}-25 \mathrm{BAC}$ & 1970 & 2,520 & GAS & $\mathrm{PC}$ & $02-08-91$ & 500 \\
\hline & & & & & & $03-27-91$ & 520 \\
\hline Bruington 1-A & $31 \mathrm{~N}-11 \mathrm{~W}-25 \mathrm{BCA}$ & 1977 & 5,018 & GAS & MV & $02-15-91$ & $<.005$ \\
\hline Zachary Pool 1 & $31 \mathrm{~N}-11 \mathrm{~W}-25 \mathrm{CAC}$ & 1955 & 4,890 & GAS & MV & $02-14-91$ & .9 \\
\hline Heaton LS-28 & $31 \mathrm{~N}-11 \mathrm{~W}-25 \mathrm{CAC}$ & 1972 & 2,650 & GAS & PC & $02-14-91$ & $<.005$ \\
\hline Heaton LS-26 & $31 \mathrm{~N}-11 \mathrm{~W}-25 \mathrm{DAC}$ & 1971 & 2,743 & GAS & PC & $02-13-91$ & $<.005$ \\
\hline
\end{tabular}


Table 3. Gas-well records and maximum methane concentrations measured in soil gas adjacent to gas-well casings--Continued

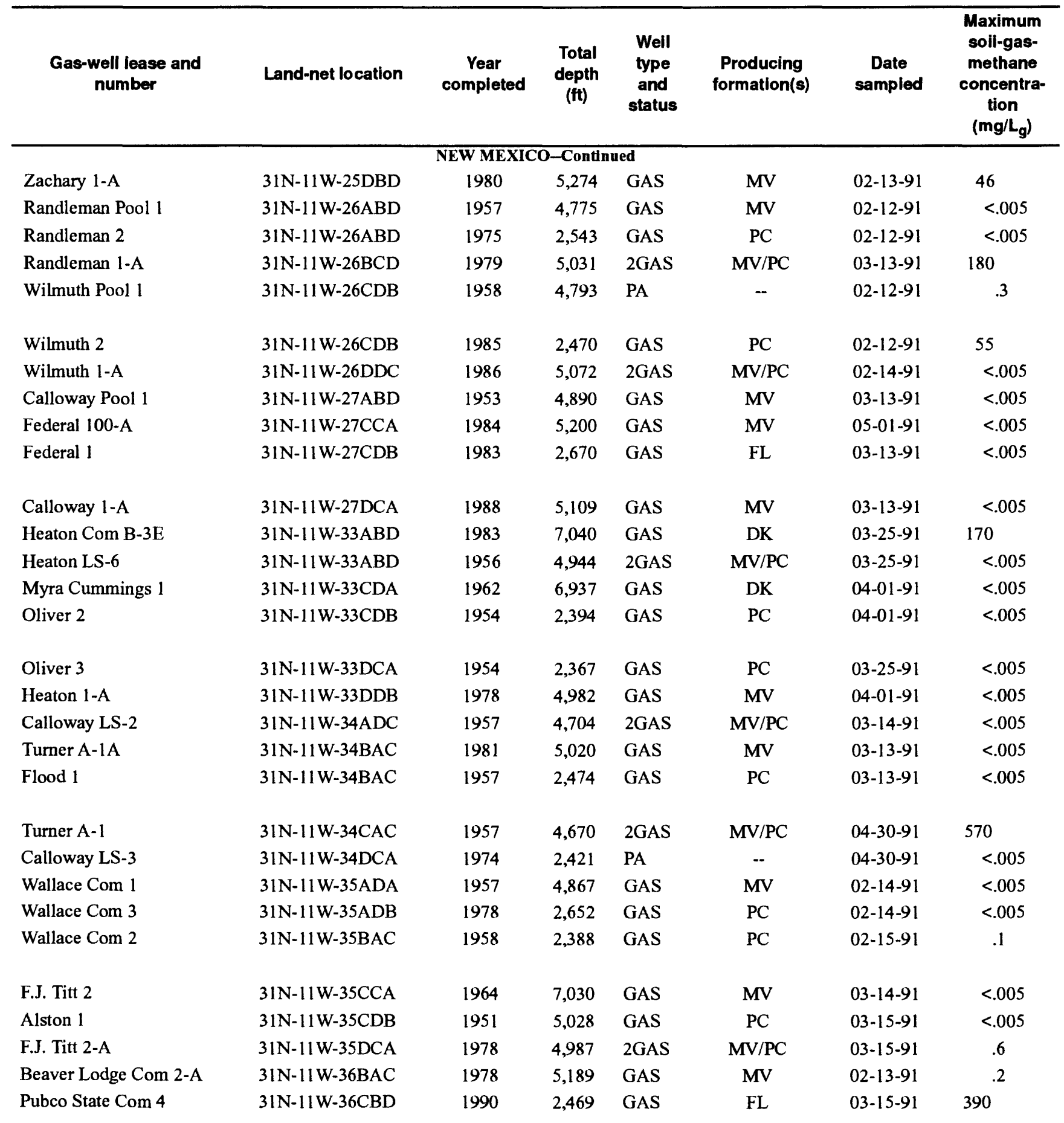


Tabie 3. Gas-well records and maximum methane concentrations measured in soil gas adjacent to gas-well casings--Continued

\begin{tabular}{|c|c|c|c|c|c|c|c|}
\hline $\begin{array}{c}\text { Gas-well lease and } \\
\text { number }\end{array}$ & Land-net locatlon & $\begin{array}{c}\text { Year } \\
\text { completed }\end{array}$ & $\begin{array}{l}\text { Total } \\
\text { depth } \\
\text { (ft) }\end{array}$ & $\begin{array}{l}\text { Well } \\
\text { type } \\
\text { and } \\
\text { status }\end{array}$ & $\begin{array}{l}\text { Producing } \\
\text { formation(s) }\end{array}$ & $\begin{array}{l}\text { Date } \\
\text { sampled }\end{array}$ & $\begin{array}{c}\text { Maximum } \\
\text { soil-gas- } \\
\text { methane } \\
\text { concentra- } \\
\text { tion } \\
\left(\mathrm{mg} / \mathrm{L}_{\mathrm{g}}\right)\end{array}$ \\
\hline \multicolumn{8}{|c|}{ NEW MEXICO-Continued } \\
\hline Pubco State Com 1 & $31 \mathrm{~N}-11 \mathrm{~W}-36 \mathrm{CBD}$ & 1955 & 4,860 & GAS & MV & $03-15-91$ & $<0.005$ \\
\hline Stull 1 & $32 \mathrm{~N}-10 \mathrm{~W}-10 \mathrm{CBD}$ & 1956 & 5,480 & GAS & MV & $02-04-91$ & $<.005$ \\
\hline Stull 100 & $32 \mathrm{~N}-10 \mathrm{~W}-10 \mathrm{DBD}$ & 1989 & 2,675 & GAS & $\mathrm{FL}$ & $02-04-91$ & $<.005$ \\
\hline San Juan $32-9,300$ & $32 \mathrm{~N}-10 \mathrm{~W}-11 \mathrm{CAA}$ & 1990 & 3,247 & GAS & FL & $03-08-91$ & .1 \\
\hline San Juan 32-9, 55 & $32 \mathrm{~N}-10 \mathrm{~W}-11 \mathrm{CAD}$ & 1957 & 5,900 & GAS & MV & $03-08-91$ & $<.005$ \\
\hline San Juan 32-9, 48 & $32 \mathrm{~N}-10 \mathrm{~W}-14 \mathrm{CAC}$ & 1957 & 6,030 & GAS & MV & $03-08-91$ & $<.005$ \\
\hline Heizer 1 & $32 \mathrm{~N}-10 \mathrm{~W}-15 \mathrm{ABD}$ & 1955 & 5,265 & GAS & MV & $02-04-91$ & $<.005$ \\
\hline Heizer 100 & $32 \mathrm{~N}-10 \mathrm{~W}-15 \mathrm{ACB}$ & 1989 & 2,702 & GAS & FL & $02-04-91$ & .005 \\
\hline Bonds Com 100 & $32 \mathrm{~N}-10 \mathrm{~W}-15 \mathrm{CCA}$ & 1990 & 2,717 & GAS & $\mathrm{FL}$ & $02-05-91$ & $<.005$ \\
\hline Bonds Pool 1 & $32 \mathrm{~N}-10 \mathrm{~W}-15 \mathrm{CCA}$ & 1956 & 5,320 & GAS & MV & $02-05-91$ & 22 \\
\hline Burroughs Com A-2 & $32 \mathrm{~N}-10 \mathrm{~W}-16 \mathrm{ACA}$ & 1958 & 5,754 & GAS & MV & $02-06-91$ & $<.005$ \\
\hline Burroughs Com A-100 & $32 \mathrm{~N}-10 \mathrm{~W}-16 \mathrm{ACD}$ & 1989 & 3,099 & GAS & FL & $02-06-91$ & $<.005$ \\
\hline El Paso Com C-4 & $32 \mathrm{~N}-10 \mathrm{~W}-16 \mathrm{CDB}$ & 1956 & 5,637 & GAS & MV & $02-01-91$ & $<.005$ \\
\hline Burroughs Com A-2A & $32 \mathrm{~N}-10 \mathrm{~W}-16 \mathrm{DCA}$ & 1979 & 5,825 & GAS & MV & $02-01-91$ & $<.005$ \\
\hline Payne 3 & $32 \mathrm{~N}-10 \mathrm{~W}-20 \mathrm{ACA}$ & 1954 & 5,555 & $\mathrm{PA}$ & -- & $01-28-91$ & $<.005$ \\
\hline Payne 3-E & $32 \mathrm{~N}-10 \mathrm{~W}-20 \mathrm{ADD}$ & 1990 & 7,900 & GAS & DK & $02-01-91$ & .2 \\
\hline Payne 6 & $32 \mathrm{~N}-10 \mathrm{~W}-20 \mathrm{DAC}$ & 1984 & 3,121 & GAS & FL & $01-28-91$ & 5 \\
\hline Payne 1-A & $32 \mathrm{~N}-10 \mathrm{~W}-20 \mathrm{DDB}$ & 1978 & 5,725 & GAS & MV & $01-28-91$ & $<.005$ \\
\hline Holmberg Com D-1 & $32 \mathrm{~N}-10 \mathrm{~W}-21 \mathrm{ABD}$ & 1989 & 3,300 & GAS & FL & $01-28-91$ & 280 \\
\hline Sullivan Com B-1 & $32 \mathrm{~N}-10 \mathrm{~W}-21 \mathrm{ABD}$ & 1954 & 5,610 & GAS & MV & $01-28-91$ & $<.005$ \\
\hline \multicolumn{8}{|c|}{ 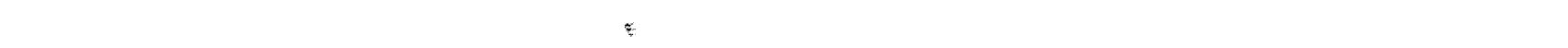 } \\
\hline Payne 2-A & $32 \mathrm{~N}-10 \mathrm{~W}-21 \mathrm{BBD}$ & 1980 & 7,800 & $2 \mathrm{GAS}$ & $\mathrm{DK} / \mathrm{MV}$ & $01-28-91$ & $<.005$ \\
\hline Payne Federal 8 & $32 \mathrm{~N}-10 \mathrm{~W}-21 \mathrm{CAC}$ & 1984 & 7,810 & GAS & FL & $01-28-91$ & $<.005$ \\
\hline Payne 2 & $32 \mathrm{~N}-10 \mathrm{~W}-21 \mathrm{CAC}$ & 1954 & 5,609 & GAS & MV & $01-28-91$ & $<.005$ \\
\hline Sullivan Com B-1A & $32 \mathrm{~N}-10 \mathrm{~W}-21 \mathrm{DDB}$ & 1977 & 5,300 & GAS & MV & $01-18-91$ & $<.005$ \\
\hline Payne 4 & $32 \mathrm{~N}-10 \mathrm{~W}-22 \mathrm{ACA}$ & 1954 & 5,750 & GAS & MV & $02-05-91$ & $<.005$ \\
\hline Sullivan Com 1-A & $32 \mathrm{~N}-10 \mathrm{~W}-22 \mathrm{BBD}$ & 1981 & 5,217 & GAS & MV & $01-14-91$ & $<.005$ \\
\hline Sullivan Com 1 & $32 \mathrm{~N}-10 \mathrm{~W}-22 \mathrm{CCA}$ & 1954 & 5,300 & GAS & MV & $01-16-91$ & $<.005$ \\
\hline Payne 4-A & $32 \mathrm{~N}-10 \mathrm{~W}-22 \mathrm{DDA}$ & 1980 & 8,400 & 2GAS & $\mathrm{DK} / \mathrm{MV}$ & 03-08-91 & 560 \\
\hline San Juan $32-9,80$ & $32 \mathrm{~N}-10 \mathrm{~W}-23 \mathrm{CCA}$ & 1973 & 3,894 & $\mathrm{DA}$ & - & 03-08-91 & $<.005$ \\
\hline San Juan 32-9, 27 & $32 \mathrm{~N}-10 \mathrm{~W}-23 \mathrm{CDB}$ & 1955 & 6,265 & GAS & MV & $03-08-91$ & $<.005$ \\
\hline
\end{tabular}


Tabie 3. Gas-well records and maximum methane concentrations measured in soil gas adjacent to gas-well casings--Continued

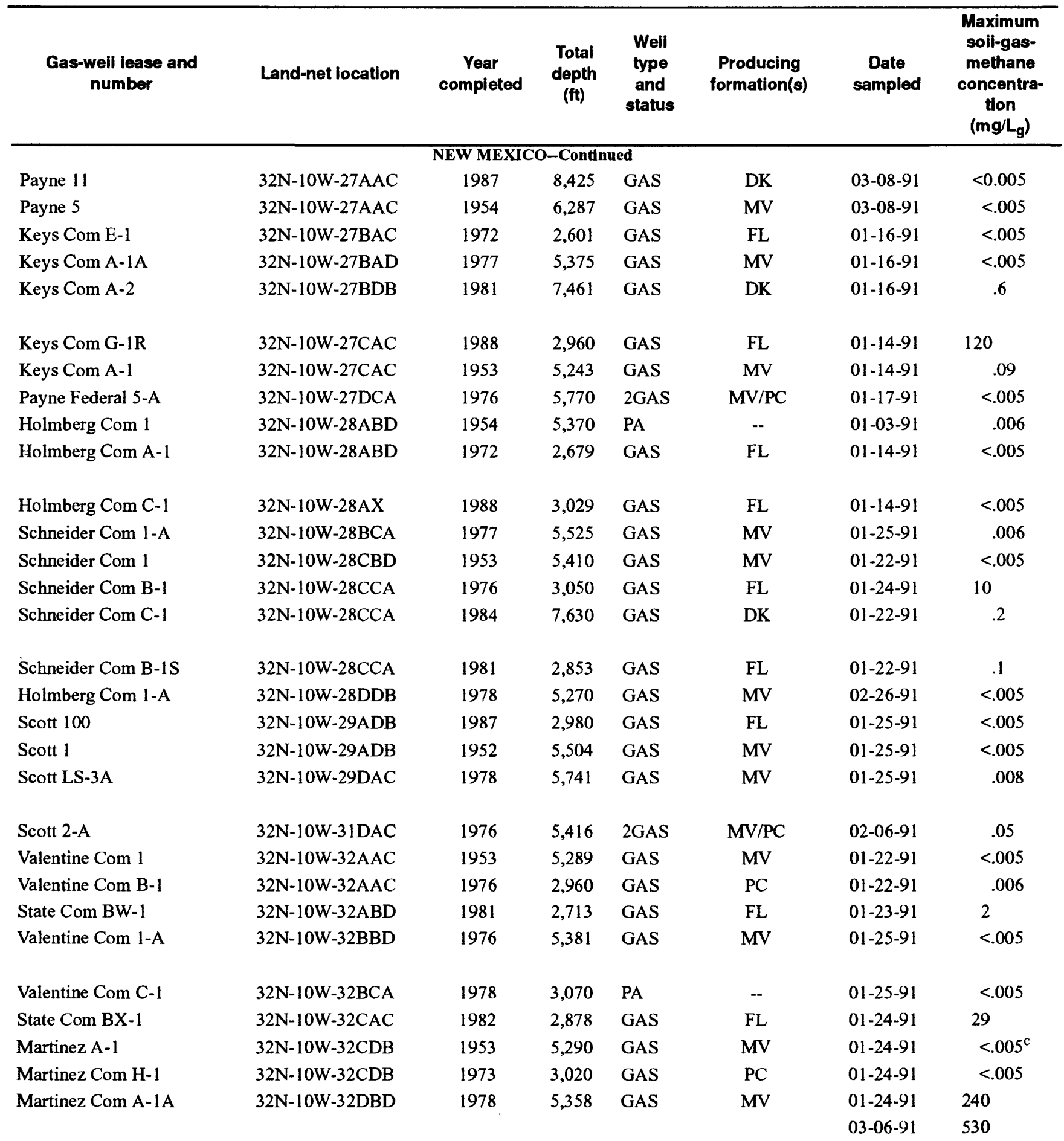


Table 3. Gas-well records and maximum methane concentrations measured in soil gas adjacent to gas-well casings--Continued

\begin{tabular}{|c|c|c|c|c|c|c|c|}
\hline $\begin{array}{c}\text { Gas-well lease and } \\
\text { number }\end{array}$ & Land-net location & $\begin{array}{c}\text { Year } \\
\text { completed }\end{array}$ & $\begin{array}{l}\text { Total } \\
\text { depth } \\
\text { (ft) }\end{array}$ & $\begin{array}{l}\text { Well } \\
\text { type } \\
\text { and } \\
\text { status }\end{array}$ & $\begin{array}{l}\text { Producing } \\
\text { formation(s) }\end{array}$ & $\begin{array}{l}\text { Date } \\
\text { sampled }\end{array}$ & $\begin{array}{c}\text { Maximum } \\
\text { soil-gas- } \\
\text { methane } \\
\text { concentra- } \\
\text { tion } \\
\left(\mathrm{mg} / \mathrm{L}_{\mathrm{g}}\right)\end{array}$ \\
\hline \multicolumn{8}{|c|}{ NEW MEXICO-Continued } \\
\hline Carr Com 1 & $32 \mathrm{~N}-10 \mathrm{~W}-32 \mathrm{DBD}$ & 1978 & 2,955 & GAS & PC & $01-23-91$ & $<0.005$ \\
\hline Ealum Com C-1R & $32 \mathrm{~N}-10 \mathrm{~W}-33 \mathrm{ACA}$ & 1987 & 2,955 & GAS & FL & $01-03-91$ & $<.05$ \\
\hline Ealum Com 1 & $32 \mathrm{~N}-10 \mathrm{~W}-33 \mathrm{ADB}$ & 1953 & 5,320 & PA & -. & $01-03-91$ & $<.005$ \\
\hline Cahn Com 1 & $32 \mathrm{~N}-10 \mathrm{~W}-33 \mathrm{BAC}$ & 1977 & 2,812 & PA & -- & $01-23-91$ & $<.005$ \\
\hline Cahn Com 2 & $32 \mathrm{~N}-10 \mathrm{~W}-33 \mathrm{BCA}$ & 1976 & 2,946 & PA & -- & $01-23-91$ & $<.005$ \\
\hline Uptegrove Com 1 & $32 \mathrm{~N}-10 \mathrm{~W}-33 \mathrm{CBD}$ & 1953 & 5,270 & GAS & MV & $01-22-91$ & $<.005$ \\
\hline Gardner Com 1 & $32 \mathrm{~N}-10 \mathrm{~W}-33 \mathrm{DAC}$ & 1979 & 2,926 & PA & -- & $12-07-90$ & .006 \\
\hline Uptegrove Com 1-A & $32 \mathrm{~N}-10 \mathrm{~W}-33 \mathrm{DAC}$ & 1977 & 5,250 & GAS & MV & $12-07-90$ & $<.005$ \\
\hline Scott 18 & $32 \mathrm{~N}-10 \mathrm{~W}-34 \mathrm{AAC}$ & 1977 & 3,154 & PA & - & $04-02-91$ & $<.005$ \\
\hline Scott 102 & $32 \mathrm{~N}-10 \mathrm{~W}-34 \mathrm{AAC}$ & 1988 & 3,005 & GAS & FL & $12-14-90$ & $<.005$ \\
\hline Scott 5 & $32 \mathrm{~N}-10 \mathrm{~W}-34 \mathrm{ADA}$ & 1954 & 5,315 & GAS & MV & $12-14-91$ & $<.005^{d}$ \\
\hline Leeper Com B-1 & $32 \mathrm{~N}-10 \mathrm{~W}-34 \mathrm{BAD}$ & 1977 & 2,851 & PA & -- & $03-27-91$ & 1 \\
\hline Leeper Com 1 & $32 \mathrm{~N}-10 \mathrm{~W}-34 \mathrm{CDB}$ & 1953 & 5,065 & GAS & MV & $12-07-90$ & $<.005$ \\
\hline Scott 5-A & $32 \mathrm{~N}-10 \mathrm{~W}-34 \mathrm{DAD}$ & 1977 & 5,464 & GAS & MV & $12-14-90$ & $<.005$ \\
\hline Scott 19 & $32 \mathrm{~N}-10 \mathrm{~W}-34 \mathrm{DCA}$ & 1976 & 3,022 & GAS & PC & $01-17-91$ & $<.005$ \\
\hline
\end{tabular}

\footnotetext{
${ }^{a}$ Maximum concentration measured adjacent to cathodic-protection well was 560 milligrams per liter of gas.

${ }^{b}$ Maximum concentration measured adjacent to cathodic-protection well was 7 milligrams per liter of gas.

${ }^{\mathrm{c}}$ Maximum concentration measured adjacent to cathodic-protection well was 10 milligrams per liter of gas.

${ }^{\mathrm{d}}$ Maximum concentration measured adjacent to cathodic-protection well was 30 milligrams per liter of gas.
} 


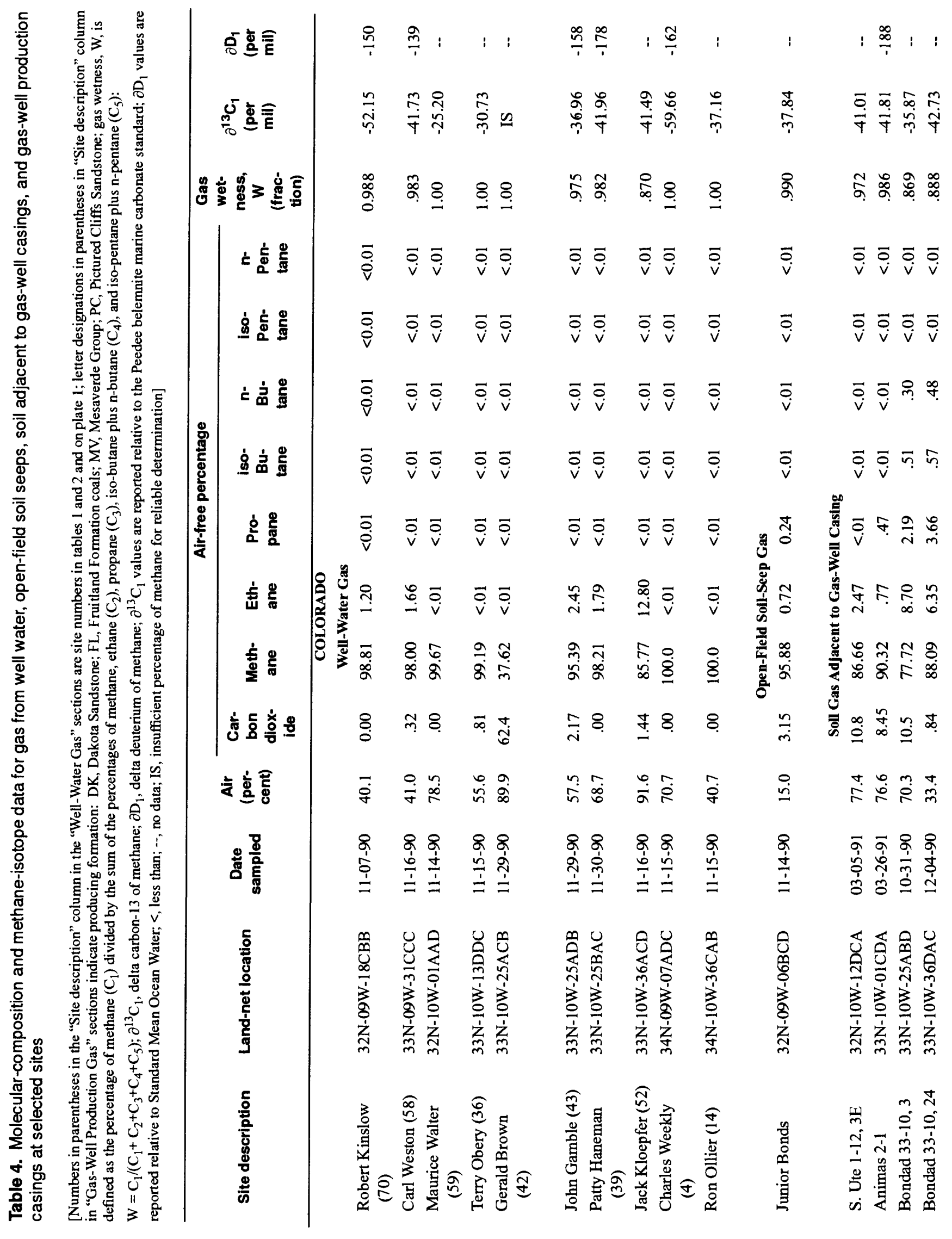




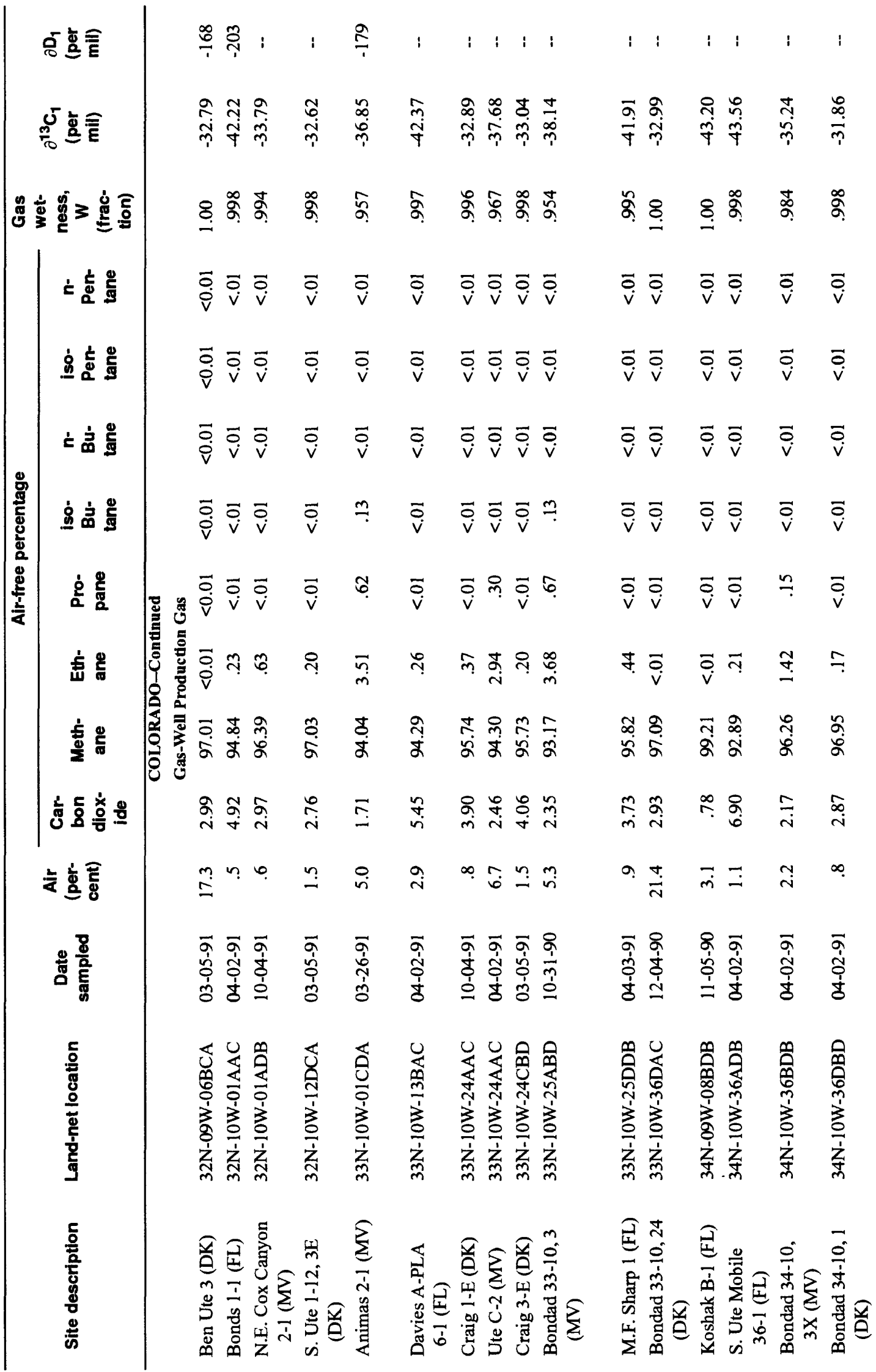




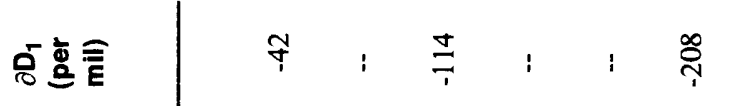

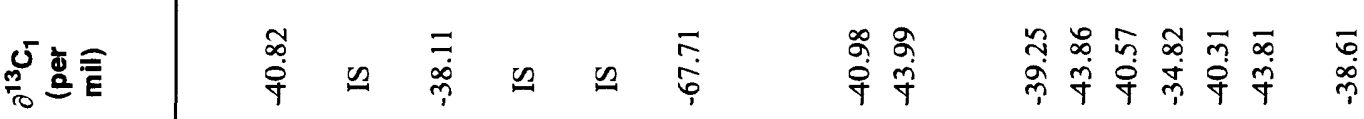

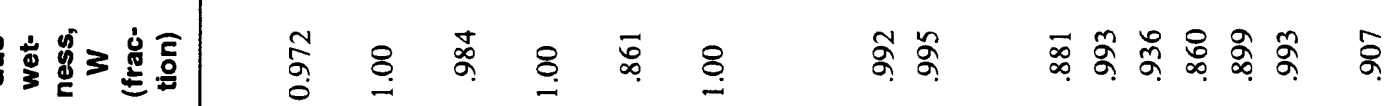

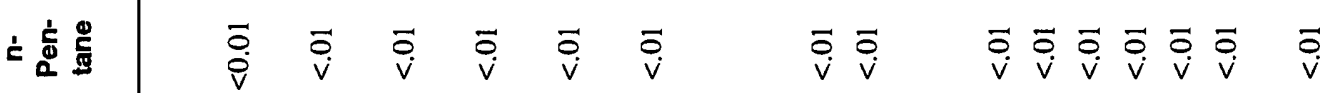

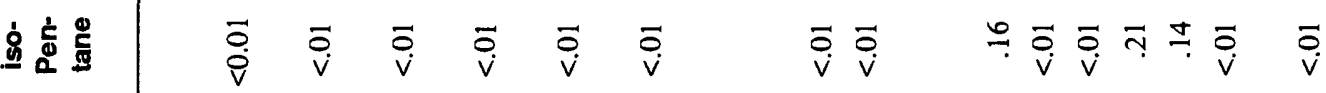

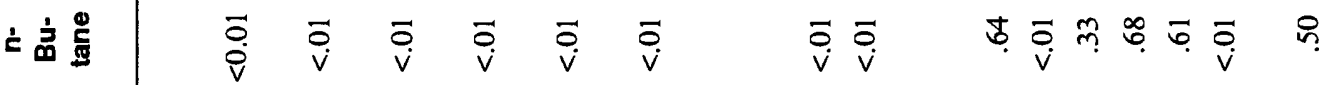

i்

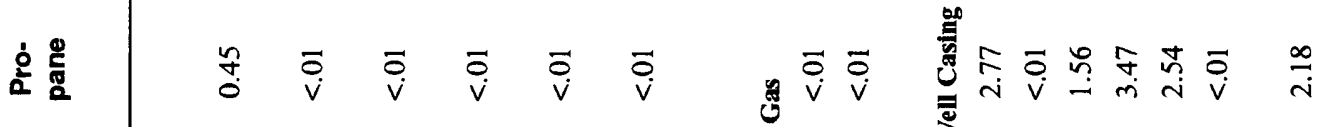

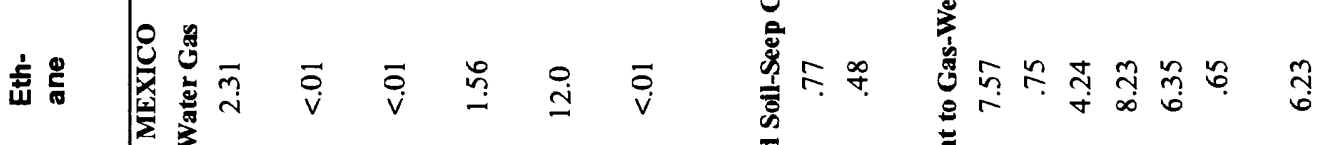

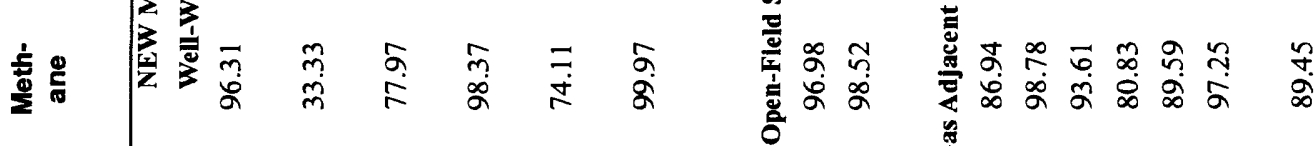

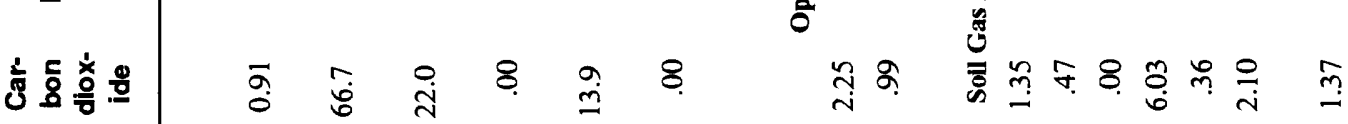

を产焉 守

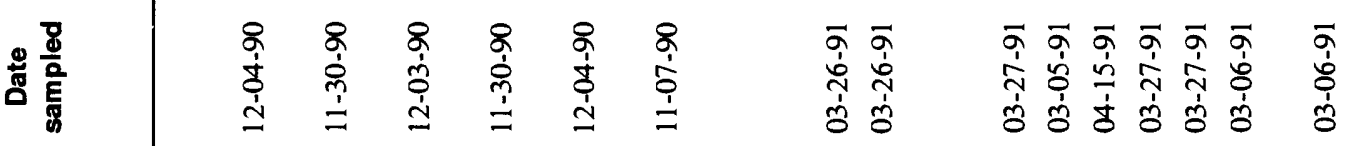

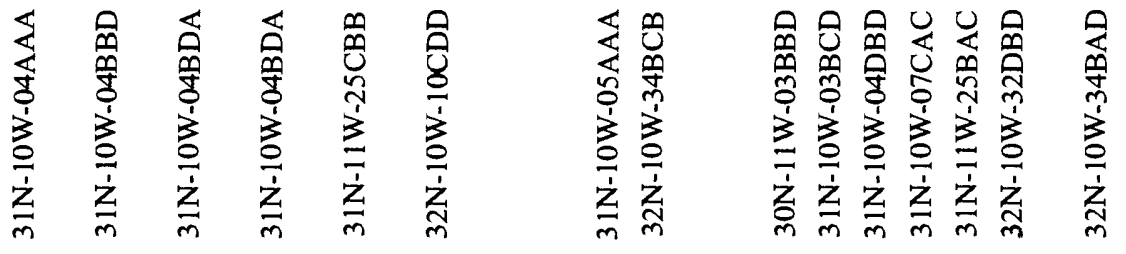

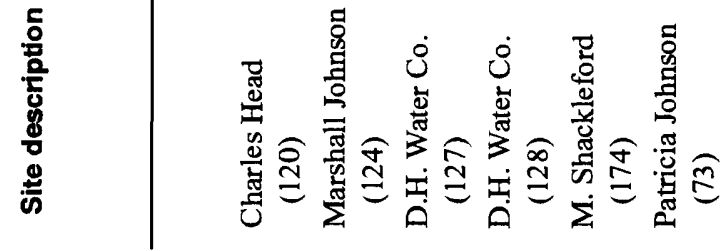

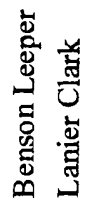

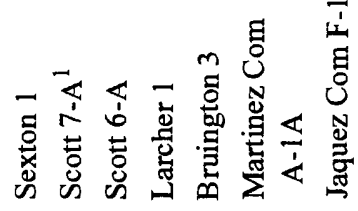




\section{SUMMARY}

Shallow ground water obtained from alluvium and Tertiary sedimentary rocks overlying Cretaceous gas-bearing formations in the northwestern part of the San Juan Basin locally is contaminated by methane. Recent development of coal-bed methane from the Cretaceous Fruitland Formation has caused public concern about the possibility of increasing contamination of shallow ground water, especially in the Animas River Valley, one of the most populated areas in the San Juan Basin.

In July 1990, the U.S. Geological Survey began a study of methane contamination of ground water in the Animas River Valley between Durango, Colorado, and Aztec, New Mexico. The purpose of the study is to identify the possible sources of and migration pathways for methane in shallow ground water in the study area. This report addresses the data-collection phase of the study and presents data collected by the U.S. Geological Survey mostly during August 1990May 1991.

Results of analyses of water samples from 203 wells and 2 springs were used to map the presence of methane in ground water, to determine relations between methane concentrations and other factors, and to select water samples for molecular and methaneisotope analyses of headspace gas. Soil-gas-methane concentrations were measured at 192 ground-water sites, by the casings of 352 gas wells (and some associated cathodic-protection wells) within $0.5 \mathrm{mi}$ of the Animas River Valley, and at 4 open-field soil seeps. Gas from 16 water samples, from 3 soil seeps in open fields, from 10 soil columns adjacent to gas-well casings and 1 column adjacent to a cathodic-protection well, and from 30 gas wells were collected for analyses of molecular composition and carbon- 13 content of methane.

Methane concentrations were measured onsite with an organic gas analyzer equipped with a gaschromatograph column capable of separating methane and ethane. Onsite calibration checks indicated that the overall variation in accuracy was about \pm 20 percent.

Selected gas samples were analyzed for concentrations of air components, hydrocarbons, and carbon dioxide by the U.S. Geological Survey in Denver, Colorado, by means of thermal-conductivity gas chromatography. Some of these samples also were analyzed for methane hydrogen-isotope ratios at a contract laboratory.

\section{REFERENCES CITED}

Fassett, J.E., and Hinds, J.S., 1971, Geology and fuel resources of the Fruitland Formation and Kirtland Shale of the San Juan Basin, New Mexico and Colorado: U.S. Geological Survey Professional Paper 676, 76 p.

Fishman, M.J., and Friedman, L.C., eds., 1989, Methods for determination of inorganic substances in water and fluvial sediments: U.S. Geological Survey Techniques of Water-Resources Investigations, book 5, chap. Al, $545 \mathrm{p}$.

Freeze, R.A., and Cherry, J.A., 1979, Groundwater: Englewood Cliffs, N.J., Prentice-Hall, Inc., 604 p. Yamamoto, Sachio, Alcauskas, J.B., and Crozier, T.E., 1976, Solubility of methane in distilled water and seawater: Journal of Chemical and Engineering Data, v. 21 , no. 1, p. $78-80$. 
